\title{
DEGRADATION OF RF MEMS CAPACITIVE SWITCHES
}

Roelof Willem Herfst 
Samenstelling promotiecommissie:

Voorzitter: prof.dr.ir. A.J. Mouthaan Universiteit Twente

Secretaris: prof.dr.ir. A.J. Mouthaan Universiteit Twente

Promotor: prof.dr. J. Schmitz Universiteit Twente

Leden: $\quad$ prof.dr.ir. F.G. Kuper Universiteit Twente,

NXP Semiconductors

prof.dr. M.C. Elwenspoek Universiteit Twente

prof.dr. P.M. Sarro Technische Universiteit Delft

Deskundige: dr. P.G. Steeneken NXP Semiconductors

Referent: dr. I. De Wolf IMEC

R.W. Herfst

Reliability Engineer in RF MEMS capacitive switches

Ph.D. thesis, University of Twente, The Netherlands

ISBN: 978-90-365-2750-7

Cover design: Uitgeverij BOX press

(C)R.W. Herfst, 2008 


\title{
DEGRADATION OF RF MEMS CAPACITIVE SWITCHES
}

\section{PROEFSCHRIFT}

\author{
ter verkrijging van \\ de graad van doctor aan de Universiteit Twente, \\ op gezag van de rector magnificus, \\ prof.dr. W.H.M. Zijm, \\ volgens besluit van het College voor Promoties \\ in het openbaar te verdedigen \\ op woensdag 12 november 2008 om 15.00 uur
}

door

Roelof Willem Herfst geboren op 24 mei 1980 te Waalwijk 
Dit proefschrift is goedgekeurd door de promotor:

prof.dr. J. Schmitz 


\section{Contents}

1 Introduction 1

1.1 Switch operation ...................... 2

1.1.1 Switch layout . . . . . . . . . . . . . . . . 2

1.1.2 Static switch behavior ............... . . . 3

1.1.3 Calculation of $V_{\mathrm{pi}}$ and $V_{\mathrm{po}}$ in a lumped element model . . . . 4

1.1.4 Dynamic and higher order modeling . . . . . . . . . . 8

1.2 Reliability of capacitive switches . . . . . . . . . . . . . 10

1.2.1 Methodology of reliability physics . . . . . . . . . . . 11

1.2.2 Physics of MEMS reliability . . . . . . . . . . . . . 12

1.3 Conclusions and outlook . . . . . . . . . . . . . . . 15

2 Measurement methodology and equipment $\quad 17$

2.1 Introduction . . . . . . . . . . . . . . . . . 17

2.2 Measurement methodology . . . . . . . . . . . . . . . . 18

2.2.1 Methods for finding $V_{\text {shift }} \ldots \ldots \ldots$. . . . . . . . 19

2.2.2 Results: comparison of the measurement setups and methods 22

2.2.3 Measurement methodology conclusions . . . . . . . . . . . 26

2.3 Fast RF 1-port S-parameter setup . . . . . . . . . . . . . 27

2.3.1 Hardware . . . . . . . . . . . . . . . . . 28

2.3.2 Calibration of the measurement system . . . . . . . . . 31

2.3.3 Measurement automation . . . . . . . . . . . . . 37

2.4 Conclusions . . . . . . . . . . . . . . . . 38

3 Degradation mechanisms in capacitive switches 41

3.1 Introduction . . . . . . . . . . . . . . . . . . 41

3.2 Identification of degradation mechanisms . . . . . . . . . . . . . 41

3.2.1 C-V curve narrowing theory . . . . . . . . . . . . . . . . . 42

3.2.2 Measurements . . . . . . . . . . . . . . 47

3.2.3 Conclusions about identification of degradation mechanisms . 50

3.3 Homogeneous dielectric charging . . . . . . . . . . . . . . 50 
3.3.1 Measurement method and setup . . . . . . . . . . . . 51

3.3.2 Measurements and interpretation ............ 51

3.3.3 Conclusions about homogeneous dielectric charging . . . . . . 59

3.4 Inhomogeneous dielectric charging . . . . . . . . . . . . . . . 60

3.4.1 Scanning Kelvin Probe Microscopy . . . . . . . . . . . . . . . 60

3.4.2 Measurement results . . . . . . . . . . . . . . . 62

3.4.3 Estimation of the diffusion coefficient . . . . . . . . . . . 62

3.4.4 Conclusions about inhomogeneous charging . . . . . . . . . 68

3.5 Mechanical degradation . . . . . . . . . . . . . . 69

3.5.1 Characterizing mechanical degradation . . . . . . . . . . 69

3.5.2 Measurement results . . . . . . . . . . . . . . 69

3.5.3 Conclusions about mechanical degradation . . . . . . . . . 72

3.6 Conclusions . . . . . . . . . . . . . . . . . . . 74

4 Statistical aspects of capacitive switch degradation $\quad 77$

4.1 Introduction . . . . . . . . . . . . . . . . . 77

4.2 Spread in homogeneous charging rates . . . . . . . . . . . . . . . 77

4.2.1 Measurement procedure . . . . . . . . . . . . . 77

4.2 .2 Results ...................... 78

4.2.3 Conclusions on spread in homogeneous charging . . . . . . . 86

4.3 Spread in mechanical degradation . . . . . . . . . . . . . 86

4.3.1 Measurement procedure . . . . . . . . . . . 86

4.3.2 Measurement results . . . . . . . . . . . . . . . . 87

4.3.3 Conclusions on spread in mechanical degradation . . . . . . . 91

4.4 Conclusions . . . . . . . . . . . . . . . . . 92

5 Conclusions and recommendations 95

5.1 Conclusions . . . . . . . . . . . . . . . . . . . . . . 95

5.2 Recommendations ..................... 97

$\begin{array}{lr}\text { Bibliography } & 99\end{array}$

$\begin{array}{ll}\text { Summary } & 107\end{array}$

$\begin{array}{lr}\text { Samenvatting } & 109\end{array}$

$\begin{array}{ll}\text { List of publications } & 111\end{array}$

$\begin{array}{ll}\text { Dankwoord } & 113\end{array}$ 


\section{Chapter 1}

\section{Introduction}

Wireless technologies play an enormous role in today's society. The start of the wireless era can be traced back to begin more than a century ago, when scientists and inventors such as Guglielmo Marconi, Nikola Tesla, Heinrich Hertz, and Thomas Edison all played a role in the development of the wireless telegraph, soon followed by actual radio. Since then, large parts of the electromagnetic spectrum usable for communication have been filled for an ever increasing amount of applications such as radio, television, mobile phones, GPS, and WiFi. Integrating more and more of these different communication forms into a single device demands ever more flexibility and efficiency of the underlying systems.

Technology-wise this leads to a demand for tunable and switchable components with good RF characteristics and requiring little power. A promising candidate fitting this profile is the RF MEMS capacitive switch. Capacitive switches are parallel plate capacitors of which one of the two electrodes can move so that the capacitance can be switched by (changing) a bias voltage. Because the electrodes can be produced from metal instead of semiconductor materials, they have low losses, are highly linear, have a good power handling and consume little power [1]. By producing the capacitive switches on silicon wafers, mass manufacturing techniques from the semiconductor industry can be used, allowing mass production at low unit prices.

A major challenge for the successful application of these switches is achieving a high reliability. The aim of this work is to shed more light on the mechanisms involved in the degradation of capacitive switches, with an emphasis on how they influence the electrical behavior of a device.

This chapter explains how capacitive switches work and how they can be described with a relatively simple 1D mass-spring system. This model is used to introduce and describe concepts such as pull-in and pull-out voltage. These concepts are used in the subsequent chapters to model how different degradation mechanisms 


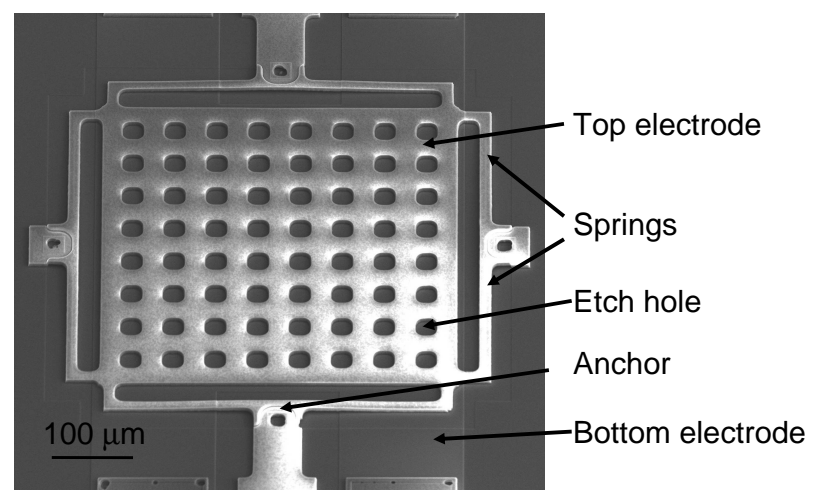

Figure 1.1: SEM picture of an RF MEMS capacitive switch. Electrodes are made of an aluminum alloy, the dielectric consists of $\mathrm{Si}_{\mathrm{x}} \mathrm{N}_{\mathrm{y}}$.

affect device behavior. Besides, an overview of previous work on the reliability of capacitive switches is given.

\subsection{Switch operation}

\subsubsection{Switch layout}

In Fig. 1.1 a SEM picture of a MEMS switch produced at NXP Semiconductors is shown. The switch (roughly $0.5 \times 0.5 \mathrm{~mm}$ ) is basically a parallel plate capacitor of which one of the plates can move. It consists of two electrodes, a dielectric layer, and an air gap. The top electrode is suspended by springs (the thin L-shaped beams connecting the top electrode to the anchors). In our case the electrode material is an aluminium alloy, and the dielectric is made of silicon nitride (not visible in the SEM picture as it is beneath the top electrode). The switch can be contacted using GSG probes with the ground pins connected to the bottom electrode and signal (which includes a DC bias voltage) pin to the upper electrode.

The switch manufacturing process is based on the Philips PASSI process [2]. The switches are made on high-ohmic silicon by depositing the different layers and structuring them with lithographic tools. During this process sacrificial layers (aluminum and $\mathrm{SiO}_{2}$ ) are also deposited (Fig. 1.2a), which are later etched away in two steps (Fig. 1.2b) to create the air gap between the top electrode and the dielectric. To make this possible, the top electrode has etch holes through which the etchant can reach the sacrificial layer. These etch holes also increase the switching speed by reducing squeeze-film damping (see section 1.1.4). The end result is a switch consisting of a bottom electrode with a thickness of $0.5 \mu \mathrm{m}$, a dielectric with 

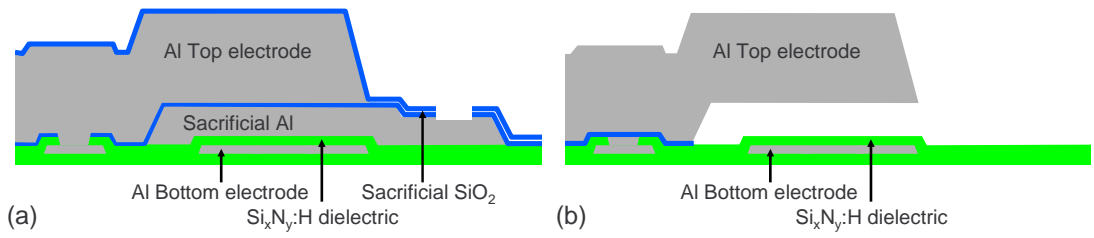

Figure 1.2: A cross section of the deposited layer stack before (a) and after sacrificial etching of first the aluminum and then the $\mathrm{SiO}_{2}$ (b).

a thickness of $0.4 \mu \mathrm{m} \mathrm{Si}_{\mathrm{x}} \mathrm{N}_{\mathrm{y}}: \mathrm{H}$, a $3.2 \mu \mathrm{m}$ air gap and an aluminum top electrode with a thickness of $5 \mu \mathrm{m}$.

\subsubsection{Static switch behavior}

Fig. 1.3 shows a schematic representation of an RF MEMS capacitive switch and the forces governing its behavior. Forces that are insignificant on the scale of the device, such as gravity, are neglected. The two electrodes form a parallel plate capacitor. At rest there is an air gap between the two electrodes with a height $g_{0}$ and a dielectric with thickness $t$ and relative dielectric permittivity $\epsilon_{r}$. When a voltage is applied to the switch, an electrostatic force $F_{E}$ will act on the top electrode. The electrode will be pulled down, increasing the capacitance between the two electrodes. Pulling down the electrode also causes a restoring spring force $F_{\text {spring }}$, which to a first order approximation is proportional to the displacement $u$.

For low voltage, a stable equilibrium $\left(-F_{E}=F_{\text {spring }}\right)$ exists (Fig. 1.4a), but at a certain voltage the balance between the attracting electrostatic force and restoring spring force becomes unstable (Fig. 1.4b) and the switch closes. When we measure the capacitance as a function of voltage (Fig. 1.5), this is marked by a sudden increase in the capacitance of the switch as the voltage is ramped up. This voltage is called the pull-in voltage $V_{\mathrm{pi}}$. The dielectric layer prevents DC current flow, but also limits the closed state capacitance by providing a repelling contact force $F_{C}$. Once the switch is closed, the electrostatic forces are much higher due to the shorter distance between the electrodes. Above $V_{\mathrm{pi}}$, the switch will move down until $F_{E}$ is in equilibrium with the sum of the contact force $F_{C}$ and spring force $F_{\text {spring }}$ (Fig. 1.4c). $F_{C}$ acts as a very stiff and nonlinear spring, and the capacitance will still increase with voltage by deforming extremities of the rough surface of the top electrode that is touching the dielectric [3]. When the voltage is lowered again, the switch will only open if the voltage is lowered below the so-called pull-out voltage $\left.V_{\text {po }}\right)$ (Fig. $1.4 \mathrm{~d}$ ), at which point the capacitance decreases. In the $C-V$ curve the fact that the switch closes at $V_{\mathrm{pi}}$ and opens at $V_{\text {po }}$ is visible as a hysteresis effect, which can be used to extract these characteristic voltages (Fig. 1.5). Later in this chapter we will see that the electrostatic force is proportional to the voltage 


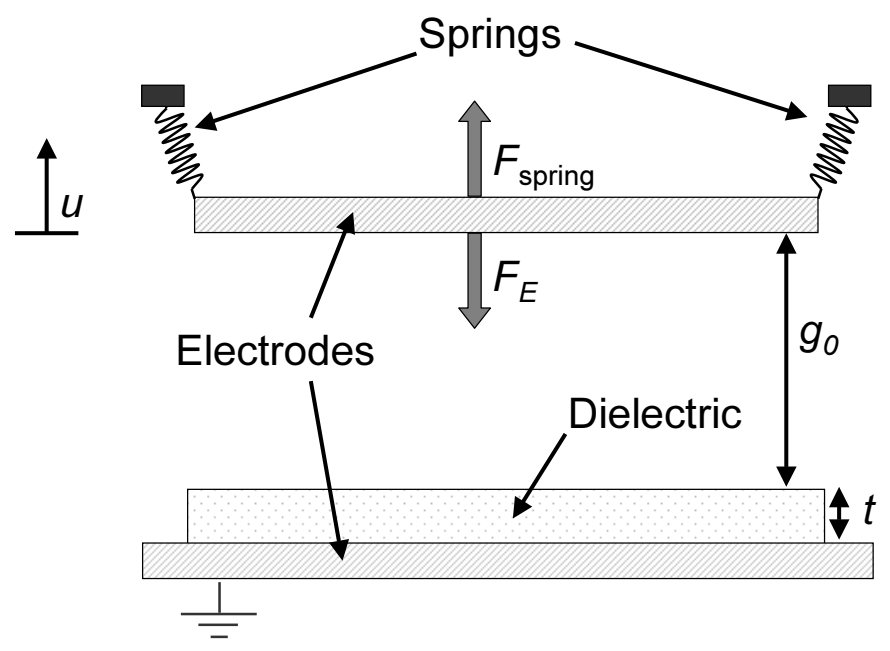

Figure 1.3: Schematic representation of an RF MEMS. The top electrode of a parallel plate capacitor can be pulled down by applying a voltage greater than the pull-in voltage $\left(|V|>\left|V_{\mathrm{pi}}\right|\right)$, which is pulled up again by the springs if the voltage is lowered beneath the pull-out voltage $\left(|V|<\left|V_{\text {po }}\right|\right)$.

squared, so that pull-in and pull-out occur for both positive and negative applied voltage, indicated in the figure by $V_{\mathrm{pi}}^{+}, V_{\mathrm{po}}^{+}, V_{\mathrm{pi}}^{-}$and $V_{\mathrm{po}}^{-}$.

\subsubsection{Calculation of $V_{\text {pi }}$ and $V_{\text {po }}$ in a lumped element model}

To model how the important switch characteristics $V_{\text {pi }}$ and $V_{\text {po }}$ depend on switch properties, the voltage at which the stable force balance becomes unstable must be calculated i.e. the situations in Fig. 1.4b and Fig. 1.4d. For this we must first know the expressions for $F_{\text {spring }}$ and $F_{E}$. Since the springs are beams that are relatively long $(\sim 100 \mu \mathrm{m})$ compared to the maximum deviation $(\sim 3 \mu \mathrm{m})$, Hooke's spring law is a good approximation so that

$$
F_{\text {spring }}(u)=-k u
$$

where $u$ is the deviation from the rest position, and $k$ the spring constant.

$F_{E}(u)$ can be found [4] by considering how much total electrical energy $U_{E}(u)$, that is the sum of the energy stored in the capacitor and the energy stored in the voltage source, changes when the top plate is moved over a infinitesimal distance $\mathrm{d} u$ :

$$
F_{E}(u)=-\frac{\mathrm{d} U_{E}(u)}{\mathrm{d} u}=-\left(\frac{\mathrm{d}}{\mathrm{d} u} \frac{1}{2} C(u) V^{2}-V \frac{\mathrm{d}}{\mathrm{d} u} Q(u)\right)
$$



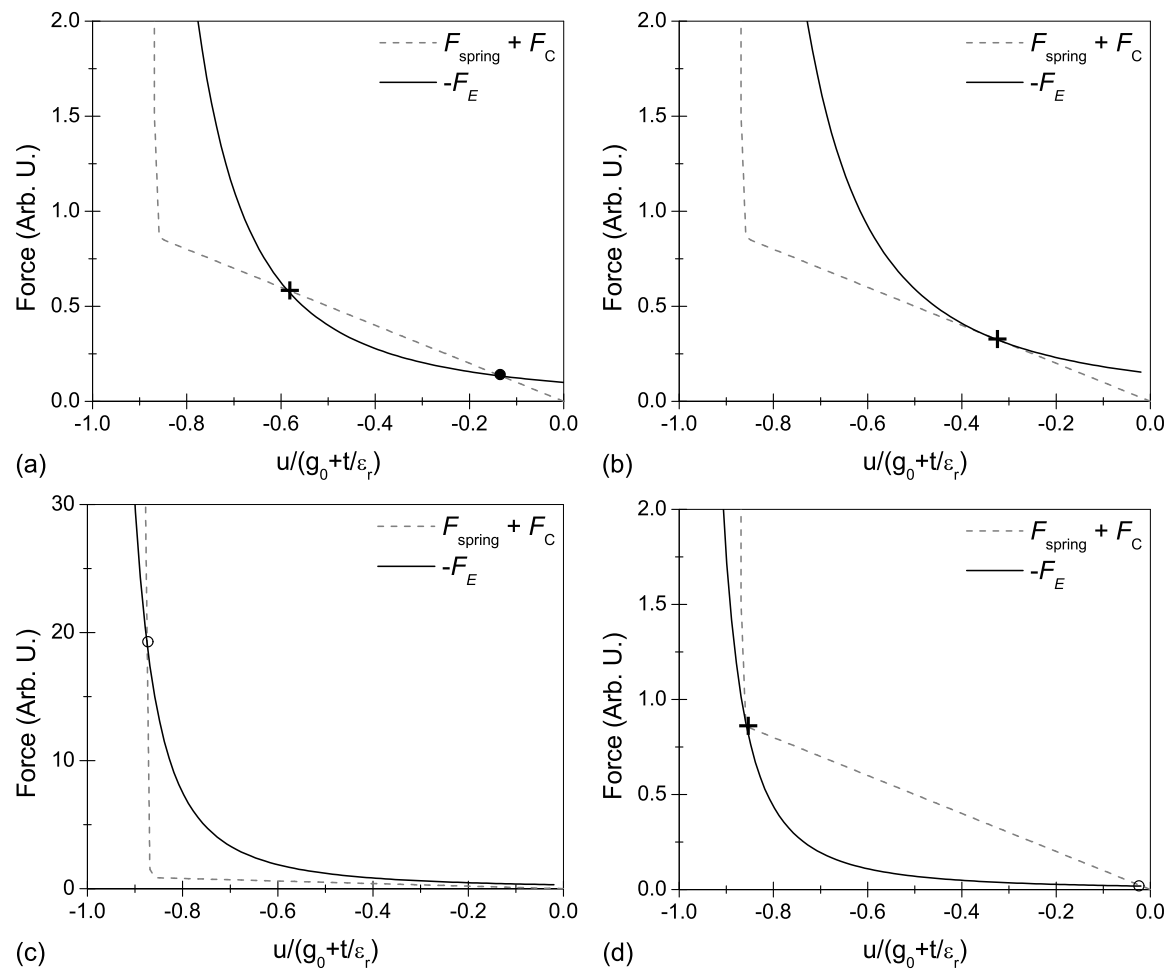

Figure 1.4: Minus electrostatic force $-F_{E}$, and spring force $F_{\text {spring }}$ plus contact force $F_{C}$ as a function of deviation $u$ from $g_{0}$ normalized to $\left(g_{0}+t / \epsilon_{r}\right)$ for different voltages. Dots indicate a stable equilibrium, pluses an unstable equilibrium. a) $V<V_{\text {pi }}$. b) $V=V_{\text {pi }}$. c) $V>V_{\text {pi }}$. d) $V=V_{\text {po. }}$. 


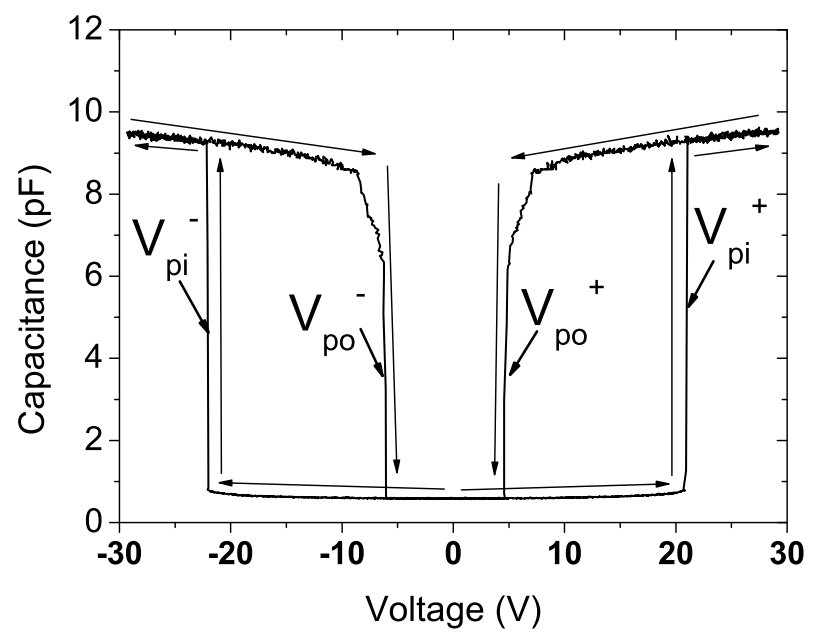

Figure 1.5: Typical $C V$ curve of an RF MEMS capacitive switch. By increasing the voltage, the top electrode is pulled down and the capacitance increases. Above $|V|=\left|V_{\mathrm{pi}}\right|$ the switch closes. When the voltage is lowered again, the switch opens for $|V|<\left|V_{\mathrm{po}}\right|$.

where $\mathrm{d} \frac{1}{2} C(u) V^{2}$ is the change in electrical electric energy stored in the capacitor, and $V \mathrm{~d} Q$ the energy the voltage source loses by providing a charge $\mathrm{d} Q$ at voltage $V$. For a parallel plate capacitor with area $A$, open state gap $g_{0}$, a dielectric with thickness $t$ and relative dielectric permittivity $\epsilon_{r}$ the capacitance is equal to

$$
C(u)=\frac{\epsilon_{0} A}{g_{0}+t / \epsilon_{r}+u} .
$$

Together with the fact that $Q=C(u) V$ we find that $F_{E}(u)$ is equal to

$$
F_{E}(u)=\frac{1}{2} V^{2} \frac{\mathrm{d} C(u)}{\mathrm{d} u}=-\frac{1}{2} \frac{\epsilon_{0} A V^{2}}{\left(g_{0}+t / \epsilon_{r}+u\right)^{2}}
$$

At any DC bias, at equilibrium the sum of the forces is zero. At pull-in however, not only is the sum of $F_{E}(u)$ and $F_{\text {spring }}(u)$ equal to zero, but the derivative of $F_{E}(u)+F_{\text {spring }}(u)$ as well (Fig. 1.4b). To calculate $V_{\text {pi }}$ we must therefore solve two equations with two unknowns:

$$
\begin{aligned}
-\frac{1}{2} \frac{\epsilon_{0} A V^{2}}{\left(g_{0}+t / \epsilon_{r}+u\right)^{2}}+-k u & =0 \\
\frac{\epsilon_{0} A V^{2}}{\left(g_{0}+t / \epsilon_{r}+u\right)^{3}}-k & =0 .
\end{aligned}
$$


Eliminating $k$ results in

$$
\left(g_{0}+t / \epsilon_{r}+u\right)^{3}=-2\left(g_{0}+t / \epsilon_{r}+u\right)^{2} u,
$$

from which we find that at pull-in the plate is positioned at $2 / 3$ of the electrical distance between the plates:

$$
u=-\frac{1}{3}\left(g_{0}+t / \epsilon_{r}\right) .
$$

Filling Eq. 1.8 into Eq. 1.6 we find that $V_{\mathrm{pi}}$ is equal to

$$
V_{\mathrm{pi}}^{ \pm}= \pm \sqrt{\frac{8 k}{27 \epsilon_{0} A}\left(g_{0}+t / \epsilon_{r}\right)^{3}},
$$

where $V_{\mathrm{pi}}^{+}$is the positive pull-in voltage and $V_{\mathrm{pi}}^{-}$the negative pull-in voltage. Of course this equation only holds when $g_{0}$ is large enough that there is an unstable equilibrium, so that $g_{0}$ must be at least $0.5 \cdot t / \epsilon_{r}$.

To calculate the pull-out voltage, we also have to consider the contact force. In principle this requires a modeling of the contact force [3], but an approximate value can be found if we assume that the contact involves two infinitely hard surfaces. In this case pull-out happens when the electrostatic force is equal to the spring force at $u=-g_{0}$, so that $V_{\text {po }}$ can be found by filling in $u=-g_{0}$ into 1.5 , resulting in

$$
V_{\mathrm{po}}^{ \pm}= \pm \sqrt{\frac{2 k}{\epsilon_{0} A}\left(t / \epsilon_{r}\right)^{2} g_{0}} .
$$

In practice this equation does not hold very precisely due to the already mentioned nonlinear contact force, as well as the fact that the membrane is not perfectly stiff. At voltages that are significantly above $V_{\mathrm{pi}}$ this has no large effect on the $C-V$ curve as the whole membrane will be pulled down. However, at lower voltages the springs will cause the membrane to bend away from the dielectric at the points where the springs are attached to the membrane (roll-off effects). This will change the value of $V_{\text {po }}$. The roll-off effects are also visible in the $C-V$ curve (Fig. 1.5) in the closed-state region between $V_{\mathrm{po}}$ and $V_{\mathrm{pi}}$ where the capacitance of a perfectly stiff membrane would remain high just before pull-out (no round corners). These effects can be incorporated in higher dimensional models.

Another important figure of merit is the switching ratio. This is defined as the closed state capacitance $C_{\text {close }}$ divided by the open state capacitance $C_{\text {open }}$ and needs to be high in order to be useful for RF applications. Again, this can only be calculated analytically for infinitely hard surfaces, where it is given by

$$
\frac{C_{\text {close }}}{C_{\text {open }}}=\frac{C\left(-g_{0}\right)}{C(0)}=\frac{g_{0}+t / \epsilon_{r}}{t / \epsilon_{r}}=\frac{g_{0} \epsilon_{r}}{t}+1 .
$$


The switching ratio can be increased by decreasing the thickness of the dielectric. However this will also decrease the $V_{\mathrm{po}} / V_{\mathrm{pi}}$ ratio, which we get from Eq. 1.9 and Eq. 1.10:

$$
\frac{V_{\mathrm{po}}}{V_{\mathrm{pi}}}=\sqrt{\frac{27}{4} \frac{\left(t / \epsilon_{r}\right)^{2} g_{0}}{\left(g_{0}+t / \epsilon_{r}\right)^{3}}}=\sqrt{\frac{27}{4} \frac{\left(\frac{t}{g_{0} \epsilon_{r}}\right)^{2}}{\left(1+\frac{t}{g_{0} \epsilon_{r}}\right)^{3}}}
$$

From these two equations we can see that the higher the switching ratio, the lower $V_{\text {po }} / V_{\text {pi }}$ ratio. In later chapters we will see that a low $V_{\text {po }}$ can be disadvantageous for reliability, so that a compromise between reliability and switch performance must be made. In the limit that $t \ll g_{0} \epsilon_{r}$ the impact of dielectric thickness becomes even clearer, as the $V_{\text {po }} / V_{\text {pi }}$ ratio then becomes inversely proportional to the capacitance ratio:

$$
\frac{V_{\mathrm{po}}}{V_{\mathrm{pi}}}=\sqrt{\frac{27}{4} \frac{\left(\frac{t}{g_{0} \epsilon_{r}}\right)^{2}}{\left(1+\frac{t}{g_{0} \epsilon_{r}}\right)^{3}}} \simeq \sqrt{\frac{27}{4}} \frac{t}{g_{0} \epsilon_{r}} \simeq \sqrt{\frac{27}{4} \frac{C_{\mathrm{open}}}{C_{\text {close }}}}
$$

\subsubsection{Dynamic and higher order modeling}

In the previous section, only static behavior of MEMS switches was discussed using a simple 1D model. In an actual application, a more accurate description of the MEMS is desirable, and device characteristics depending on dynamic behavior, such as the opening and closing times, are also important. Multiple approaches to model this are possible. Options for this are physics-based compact modeling [5-9] and full-blown Finite Element Modeling (FEM) [10]. Generally speaking, compact modeling is computationally most efficient, therefore more suitable for circuit simulators, but is also the least detailed approach. FEM can give the most accurate results, albeit by taking much more time to calculate static and dynamic behavior.

In a physics-based compact model, the amount of degrees of freedom is kept to a minimum, while still using equations that have a physical meaning. The simple equations for $F_{E}(u)$ and $F_{\text {spring }}(u)$ in the $1 \mathrm{D}$ model from section 1.1.3 is a starting point for such a model. The dynamic behavior is governed by Newton's second law of motion:

$$
M \frac{\mathrm{d}^{2} u}{\mathrm{~d} t^{2}}=\Sigma F=F_{E}+F_{\text {spring }}+F_{C}+F_{D}
$$

where $M$ is the mass of the top plate, and $F_{D}$ is the damping force caused by the gas film that needs to be squeezed out of the region between the top electrode and the dielectric, and is called squeeze-film damping $[11,12]$. It depends on the geometry of the top electrode and the ambient pressure at which the switch is used. Although for an exact solution the compressible Navier-Stokes equations 


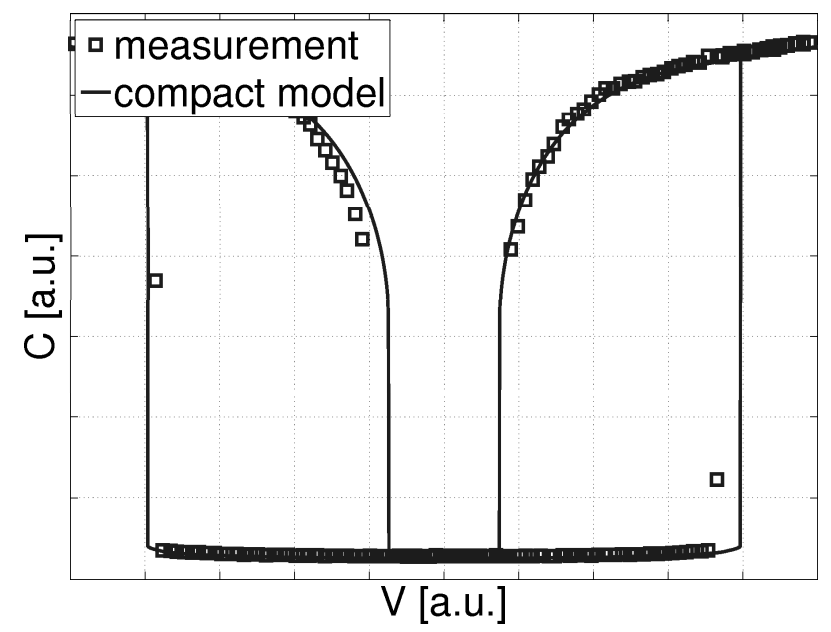

Figure 1.6: Modeled (compact) and measured $C$ - $V$-curve of an RF MEMS capacitive switch.

would have to be solved in $3 \mathrm{D}$, an approximation for $F_{D}$ can be derived from the linearized compressible Reynolds equation for gas-films $[11,13,14]$, which is given by:

$$
F_{D}(u, t)=-\frac{b}{\left(g_{0}-u\right)^{3}} \frac{\mathrm{d} u}{\mathrm{~d} t} .
$$

Here $b$ is a constant which depends on the geometry of the top plate and on the viscosity of the gas. The latter can be a function of $u$, since for low pressure and small gaps the mean free path can be on the order of the gap height, reducing the effective viscosity [15]. As can be seen, because of the damping force the differential equation is highly non-linear, so solving this problem analytically is problematic. Numerical solutions are much easier to obtain.

An implementation of a compact model in Verilog-A was written at NXP Research Eindhoven by H.M.R. Suy [5], which also includes a model for contact mechanics and the effect of fringing $E$-fields on the electrostatic force. Examples of a modeled $C-V$ curve, opening transient, and closing transient and how they compare to actual measurements (measured with the system described in chapter 2) are shown in Fig. 1.6, and 1.7. As can be seen the static $C-V$ curve is modeled quite accurately. The transient fits are not as good, but the model has enough predictive value to be useful in circuit design and simulation.

As already mentioned, more accurate results can be obtained using FEM. Using a program like Comsol or Ansys, a 3D model can be divided into a large number of small blocks (meshing). Using the appropriate equations in the mechanical 

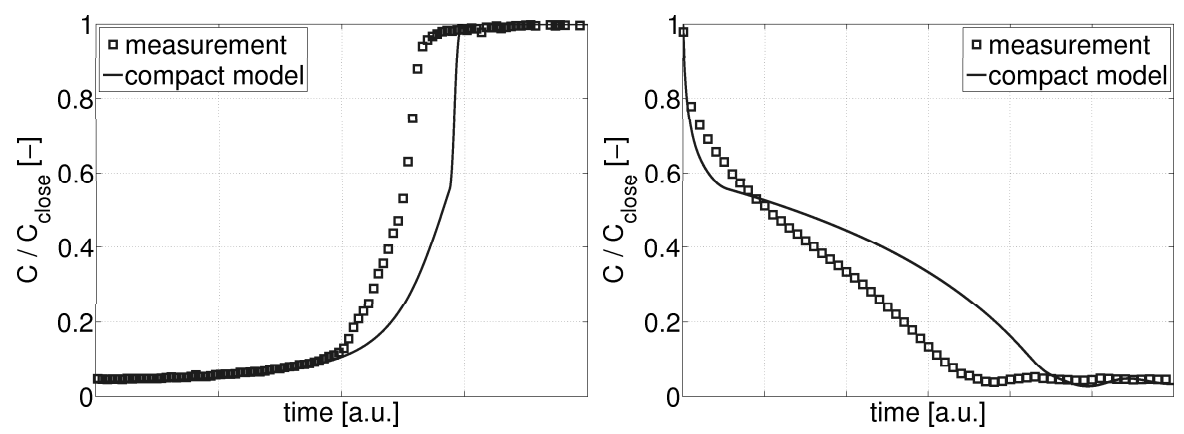

Figure 1.7: Modeled (compact) and measured closing (left) and opening (right) transient of an RF MEMS capacitive switch.
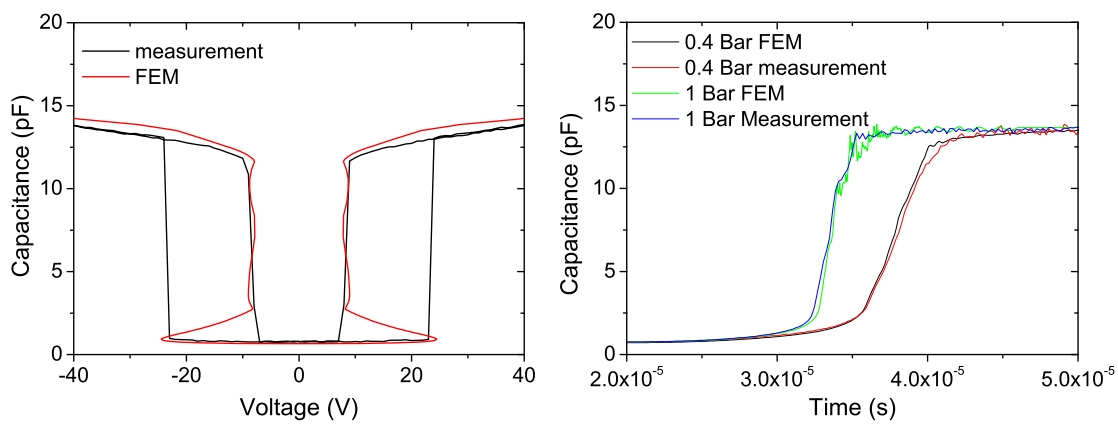

Figure 1.8: Modeled (FEM) and measured $C$ - $V$-curve (left) and closing transient (right) of an RF MEMS capacitive switch.

and electrical domain, accurate prediction of the $C-V$ curve is possible. Also, the squeeze-film damping can be described using the general 3D compressible Navier Stokes equations instead of the linearized Reynolds equation, so that transients are also more accurately predicted. An example of this is shown in Fig. 1.8. Note that the FEM model displayed here also calculates unstable solution, which leads to the $C$ - $V$ curve bending back at $V=V_{\mathrm{pi}}$, and the "wiggling" of the curve around $V=V_{\mathrm{po}}$.

\subsection{Reliability of capacitive switches}

As already mentioned, a major challenge for the successful application of RF MEMS capacitive switches is achieving a high reliability. Reliability physics of capacitive switches is still in an early stage, but a lot can be learned from the methodologies 
of reliability physics in the semiconductor industry, which is much more mature. Also, some of the actual mechanisms that are involved in the degradation of capacitive switches are quite similar to the ones present in semiconductor devices. In this section we will briefly describe the methodology of reliability physics in the semiconductor industry, as well as a short overview of the known mechanisms involved in capacitive switches.

\subsubsection{Methodology of reliability physics}

In the semiconductor industry, integrated circuits are composed of a large number of very small transistors connected by thin metal lines, which each need to work properly if the IC is going to work. It therefore needs to be proven that none of these individual transistors, as well as the metal lines connecting the transistors, will fail during the lifetime of the IC. Reliability of ICs involves process reliability and product reliability. The first typically focuses on mechanisms affecting individual transistors and interconnect lines, and typically involves intrinsic failure mechanisms. Product reliability focuses more on mechanisms that affect the product as a whole and tends to be more focused on extrinsic failure mechanisms (for instance ESD, latchup, effect of particles).

Since MEMS capacitive switches are still in a phase where the device itself is in development, we will emphasize in the rest of this thesis on process reliability of the switches. In the semiconductor industry, proving process reliability involves the following aspects:

- Identification of the (significant) failure and wear out mechanisms of individual devices.

- Modeling and measuring the influence of stress conditions (for instance voltage and temperature) on the device performance.

- Modeling and measuring the influence of spread (both intrinsic and proces induced) on the expected reliability.

All of these aspects are objects of study in itself, and involve both measurements and modeling of the devices. Examples of failure and wear out mechanisms are Negative Bias Temperature Instability (NBTI), time dependent dielectric breakdown (TDDB), electromigration, and hot carrier degradation [16]. They can be identified by (the change in) electrical device behavior, as well as a wide array of failure analysis techniques. Examples here are Scanning Electron Microscopy, Focused Ion Beam, and photo-emission.

Modeling and measuring the influence of stress conditions are needed because this enables the use of accelerated lifetime tests. Predicting failure would otherwise involve testing a large number of products for the duration of a very long time (as long as the target lifetime), which is highly impractical. By increasing stress 
parameters like the bias voltage, temperature, humidity, etc. the failure rate can be increased drastically. Provided that the degradation mechanisms stay the same and the acceleration is parameterized, the results from tests at elevated stress conditions can be extrapolated to lower temperatures and voltages to predict the reliability at the intended operating conditions.

Since in a manufacturing process there will always be some spread, the effect it has on reliability must be investigated. An example of how spread can affect device degradation in semiconductor devices is gate oxide thickness variation: devices with a thinner oxide will have a lower dielectric breakdown voltage and will be more susceptible to NBTI. Depending on the degradation mechanism, this spread in device properties will lead to a certain failure time distribution. Common distributions are the normal, log-normal, and Weibull distributions [16]. On a product reliability level, the failure rate (probability for a product to fail per hour) typically has a bathtub shaped curve, where there is initial infant mortality due to quality problems (for instance small particles), after which the failure rate reduces to a much lower level. At the end of its useful life the failure rate increases again due to the intrinsic wear out mechanisms.

In the case of MEMS reliability, most published work tends to address the first two of the three mentioned aspects of process reliability, which are of course most important during earlier stages of development. However, often MEMS processes are not very mature, so that disregarding process induced spread and the effect it has on reliability will hamper efforts to determine the influence of stress conditions on devices. In this thesis we therefore will also investigate the influence of spread effects to be able to get statistically significant results (chapter 4).

\subsubsection{Physics of MEMS reliability}

Since in general MEMS behavior is governed by multiple physical domains, MEMS reliability is also governed by many different degradation mechanisms. An overview of reliability issues in MEMS devices can be found in a publication of NASA and JPL [17]. In table 1.1 the most common reliability issues in MEMS structures are shown. Our experience is that for MEMS capacitive switches studied in this thesis, some of these issues are more important than others: since the moving structures of a capacitive switch do not slide over each other, wear is not a big issue. Also, due to the surface roughness of the bottom side of the top electrode the van der Waals forces tend to be fairly small so that stiction seems not a big issue. Environmentally induced failure is an issue, especially humidity and ESD. In this work however, we will concentrate mainly on the failure modes mechanical fracture and creep and the degradation of the dielectric.

Most literature regarding capacitive switches focuses on dielectric charging [1826], with studies on dielectrics such as silicon dioxide, silicon nitride, aluminum nitride, and silicon oxynitride. The main effect of dielectric charging is that it 
Table 1.1: Reliability issues in MEMS structures [17].

\begin{tabular}{ll}
\hline Failure mode & Underlying causes / Examples \\
\hline \multirow{3}{*}{ Mechanical fracture and creep } & Mechanical stress above Yield strength \\
& Intrinsic mechanical stress \\
& Thermal fatigue \\
\hline \multirow{2}{*}{ Degradation of dielectrics } & Dielectric charging \\
& Breakdown \\
& Leakage \\
\hline \multirow{2}{*}{ Stiction } & Van der Waals forces \\
& Capillary forces \\
\hline \multirow{2}{*}{ Wear } & Adhesion \\
& Abrasion \\
& Corrosion \\
\hline Delamination & Loss of adhesion between material interfaces \\
\hline & Vibration \\
& Shock \\
& Humidity effects \\
Environmentally induced & Radiation \\
& Particulates \\
& Temperature changes \\
& Electrostatic discharge \\
&
\end{tabular}




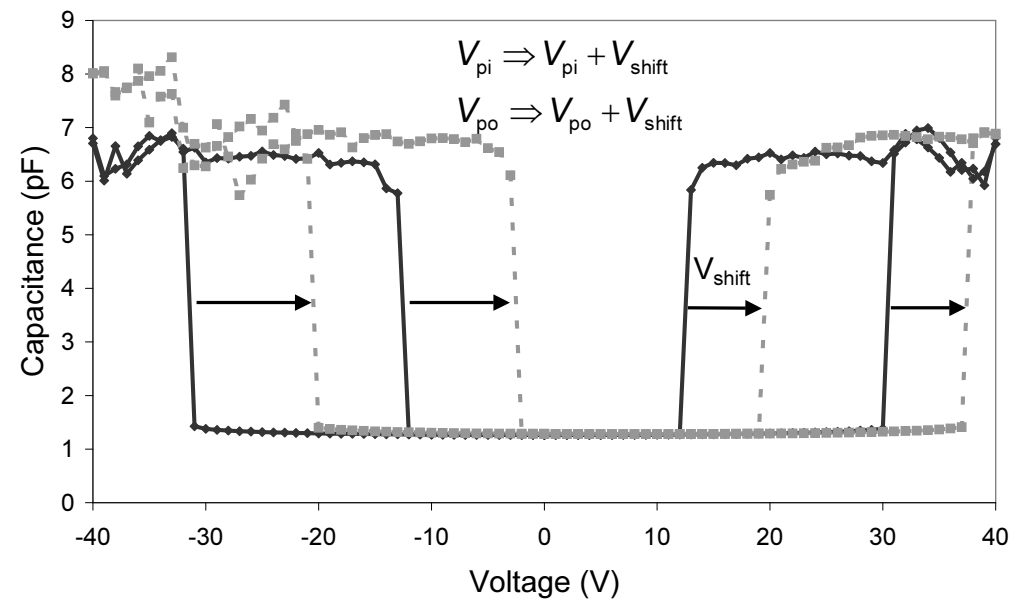

Figure 1.9: $C$ - $V$ curve before (black) and after (grey) a switch has been stressed at 65 volt for 727 seconds.

results in a built-in voltage that shifts the entire $C$ - $V$ curve $[21,24,27,28]$, which is similar to the shift of threshold voltage of MOSFETs in NBTI. The shift in the $C-V$ curve means that the pull-in and pull-out voltages also change: $V_{\mathrm{pi}} \Rightarrow V_{\mathrm{pi}}+V_{\text {shift }}$ and $V_{\text {po }} \Rightarrow V_{\text {po }}+V_{\text {shift }}$ (Fig. 1.9). Here $V_{\text {shift }}$ is linearly proportional to the amount of injected charge. A large amount of injected charge can even lead to the situation where switches fail to open when the voltage is set to zero. This happens when $V_{\text {po }}^{-}$becomes positive (due to trapping of positive charge) or when $V_{\text {po }}^{+}$becomes negative (due to trapping of negative charge). In that case the switch can be in the closed branch of the hysteresis curve even at $0 \mathrm{~V}$. For instance, if $V_{\text {po }}^{-}>0$, and the switch is closed by applying a voltage above $V_{\mathrm{pi}}^{+}$, it will not open when the voltage is suddenly (i.e. faster than the mechanical response time) set to zero again. We will go into more details regarding dielectric charging in chapter 3 .

Mechanical degradation of MEMS capacitive switches is generally seen as less of an issue than dielectric charging [18]. This is due to fact that a carefully designed switch operated under the right circumstances can operate for billions of cycles $[29,30]$. This is especially true if strong materials are used. However, reliability testing of digital micromirror devices (DMD) has shown that hinges of a MEMS device can deform under prolonged usage [31]. Since the capacitive switches that are studied here are made of the same material as these DMDs (aluminum), similar problems can be expected. We will therefore discuss how mechanical degradation can be identified (section 3.2), as well as characterize it under various circumstances (section 3.5). 


\subsection{Conclusions and outlook}

RF MEMS capacitive switches are parallel plate capacitors with a movable electrode. They are promising for the use in wireless communication due to their good $\mathrm{RF}$ characteristics. Important figures of merit are pull-in voltage $V_{\mathrm{pi}}$, pull-out voltage $V_{\text {po }}$ and the switching ratio. These can be predicted using $1 \mathrm{D}$ equations, compact models or finite element analysis. In the following chapters they will turn out to be useful to characterize degradation of RF MEMS switches under various types of stress. We will look at what the requirements for characterization of MEMS degradation are and discuss the developed measurement setup in chapter 2. We will use this system in chapter 3 to identify and characterize degradation mechanisms. In chapter 4 we will go into more detail regarding the impact of spread and statistical aspects of RF MEMS capacitive switch degradation.

A final note: in some of the figures in this thesis one or both axes are displayed in arbitrary units. This is not because only qualitative or uncalibrated signals were measured, but because the work presented in this thesis is part of a company research program on RF MEMS capacitive switches, and as such some data is sensitive information. If arbitrary units are used, actual values are multiplied by an obfuscation factor, which is known by the author. 


\section{Chapter 2}

\section{Measurement methodology and equipment}

\subsection{Introduction}

In this chapter the measurement methodology and system for the correct measurement of capacitive switch degradation is discussed, with an emphasis on characterizing laterally uniform dielectric charging. When studying degradation mechanisms in electronic devices, a certain type of stress profile is applied (which can be a constant voltage, a certain current, a pattern which mimics the use in application, or a stress-time profile that targets a particular degradation mechanism), and periodically the influence of this stress on the device is characterized by measuring the change in key electrical parameters of the device. The goal is to obtain enough insight into the degradation behavior to be able to extrapolate results obtained at high-intensity stress (i.e. high voltages and high temperatures) to normal operating conditions. In order for this to work well, there are two important conditions for the periodic measurements of the electrical parameters:

1. The effect of the measurement on the device degradation must be negligible compared to the effect of the stress itself.

2. The right parameter(s) must be chosen to monitor the degradation. A parameter is correctly chosen when:

(a) The parameter is related to only one root-cause,

(b) and/or the stress-profile/type is chosen in such a way that only one type of degradation occurs, 
(c) and/or changes in the measured parameters can be disentangled into the individual root-causes.

In addition to these conditions on the measurement methodology, it is important, but often difficult, to check if degradation mechanisms that are dominant under high stress condition remain the dominant mechanisms for low stress conditions.

In section 2.2 of this chapter several measurement methods are investigated to see how well they meet the two above mentioned criteria. The conventional method of characterizing charging (measuring changes in $V_{\mathrm{pi}}$ ) is compared with a new center shift method which measures the change of the voltage at which the capacitance is minimal. In this way the measurement method only uses very low test voltages, and therefore it does not influence the charge injected by the stressvoltage (i.e. it satisfies condition 1). Another advantage is that the measurement of the amount of injected charge is not influenced by changes in the width of the $C$ - $V$ curve (i.e. it satisfies condition 2), a phenomenon which is discussed in more detail in chapter 3 . These two advantages make it possible to measure dielectric charging in capacitive switches in a more accurate way.

During the testing of the different measurement methodologies, done on a system using a comparatively slow LCR-meter, it became apparent that moving to a faster measurement system might solve some of the observed characterization problems. For that a new and much faster setup for measuring the capacitance was developed, which is described in section 2.3. Because of the much faster measurement speed, it is also more versatile and can be used for many different measurement types. Comparison in section 2.2 with the slower LCR-meter based system shows this indeed solves the problem of charge injection during the measurement of the $C-V$ curve.

\subsection{Measurement methodology}

As already discussed in 1.2, applying a voltage across the dielectric of a capacitive switch for a long time can lead to reliability problems. In the closed state, the electric field in the dielectric layer is on the order of $1 \mathrm{MV} / \mathrm{cm}$. Because of this high field, charge is injected into the dielectric, which changes both the electric field present in the gap between the two plates, and the amount of charge in the bottom and top electrode. The net effect of injected charges is a shift of the $C-V$ curve $[21,24,32]$, which in turn affects the pull-in and pull-out voltages: $V_{\mathrm{pi}} \Rightarrow V_{\mathrm{pi}}+V_{\text {shift }}$ and $V_{\text {po }} \Rightarrow V_{\text {po }}+V_{\text {shift }}$ (Fig. 1.9). Here $V_{\text {shift }}$ is linearly proportional to the amount of injected charge. A large amount of injected charge can even lead to the situation where switch fails to open when the voltage is set to zero. This happens when $V_{\mathrm{po}}^{-}$ becomes positive (due to trapping of positive charge) or when $V_{\text {po }}^{+}$becomes negative (due to trapping of negative charge). In that case the switch can be in the closed branch of the hysteresis curve even at $0 \mathrm{~V}$. For instance, if $V_{\mathrm{po}}^{-}>0$, and the switch 


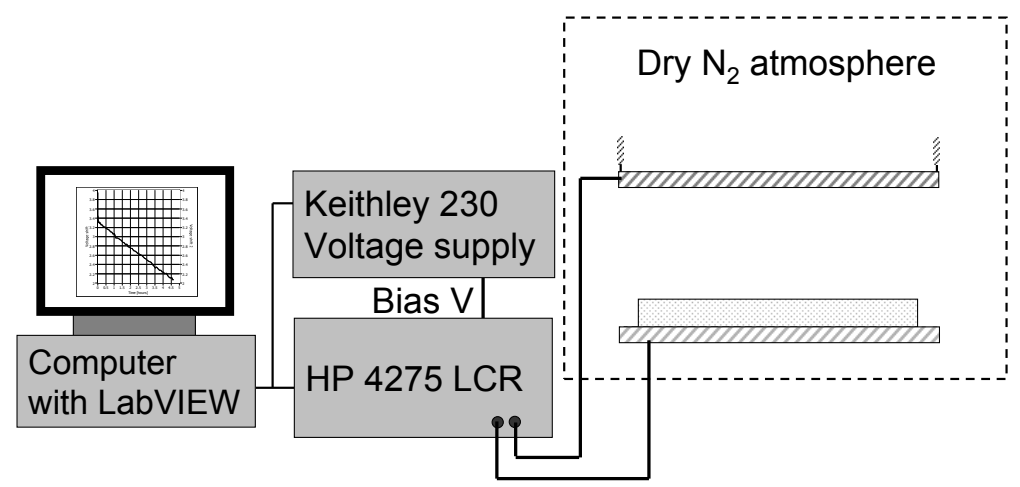

Figure 2.1: Schematic view of the LCR-based measurement setup. A LabVIEW computer program controls a HP4275 LCR meter and an Keithley 230 voltage Programmable Voltage Source. The LCR meter is connected to the RF MEMS capacitive switches, which are stressed and measured in a dry $\mathrm{N}_{2}$ atmosphere.

is closed by applying a voltage above $V_{\mathrm{pi}}^{+}$, it will not open when the voltage is suddenly (i.e. faster than the mechanical response time) set to zero again.

We will consider three different methods for measuring the shift of the $C-V$ curve with a low frequency measurement setup to investigate how well they conform to the criteria that were discussed in the introduction. Two are based on determining $V_{\mathrm{pi}}$ and one is based on determining the center shift of the $C$ - $V$ curve, which to our knowledge has not been proposed before. The influence that the measurements have on the switches is investigated and is compared with results obtained with the fast RF- $C-V$ measurement setup described in section 2.3 . The new method, in contrast to other methods, does not degrade the switches. This makes it possible to test the impact of changes in the RF MEMS manufacturing process on the reliability more accurately. The new method is used to characterize dielectric charging at several stress voltages.

\subsubsection{Methods for finding $V_{\text {shift }}$}

To study charge injection, $V_{\text {shift }}$ is measured as a function of stress voltage and time. The original setup with which these measurements were done is depicted schematically in Fig. 2.1. To avoid moisture from influencing the measurements [33], the switches are stressed and measured in a dry nitrogen environment at atmospheric pressure. A bias voltage is provided by a Keithley 230 Programmable Voltage Source to a HP4275 LCR meter which is then used to measure the capacitance as function of voltage. The test signal of the LCR meter is $4 \mathrm{MHz}$. A picture of the switches used for the measurements is shown in Fig. 2.2. 


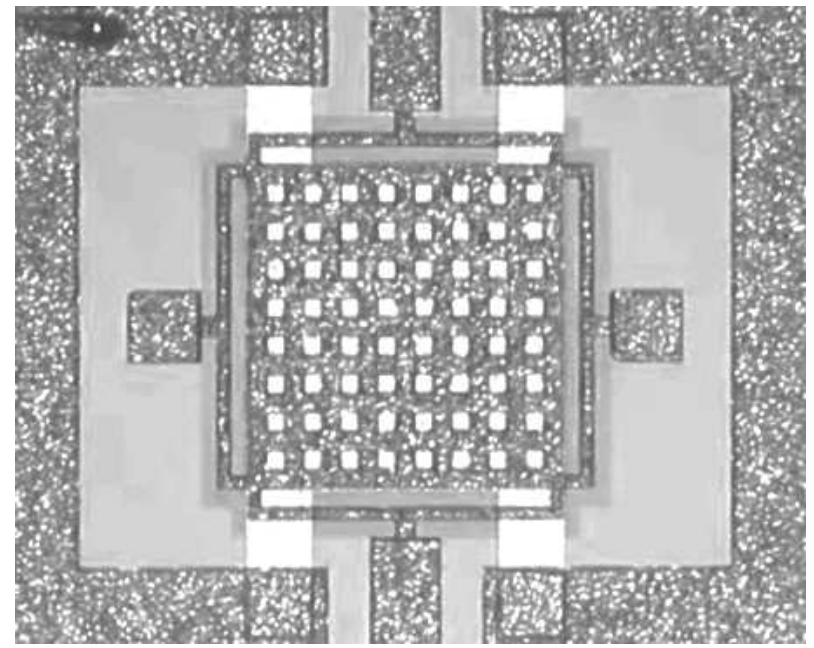

Figure 2.2: Photograph of one of the switches used in the measurements. The top electrode of the device under study is $0.46 \times 0.46 \mathrm{~mm}^{2}$, the dielectric has a thickness of approximately $0.4 \mu \mathrm{m}$, and the air gap is approximately $3.2 \mu \mathrm{m}$.

The switches are stressed and $V_{\text {shift }}$ is measured periodically. To measure $V_{\text {shift }}$, three methods are considered:

1. Whole $C-V$ curve method: Measure the $C-V$ curve with equidistant steps from below $V_{\mathrm{pi}}^{-}$to above $V_{\mathrm{pi}}^{+}$back to below $V_{\mathrm{pi}}^{-}$again. Compare to the first $C$ - $V$ curve by determining the pull-in and pull-out voltages $[19,25] . V_{\text {shift }}$ is determined by $V_{\text {shift }}=V_{\mathrm{pi}}-V_{\mathrm{pi},(t=0)}$.

2. Successive approximation method: Similar to the whole $C-V$ curve method, this method searches for the value of $V_{\mathrm{pi}}^{+}$by an algorithm based on successive approximation. The algorithm starts with an upper (closed state) and lower (open state) boundary, and applies a voltage halfway of this interval. Then the capacitance is measured and compared to $C_{\text {close }}$ and $C_{\text {open }}$, so that it can be determined if the switch is open or closed at the applied voltage. If the switch is open, the voltage becomes the new lower boundary, if it is closed, it becomes the new upper boundary. This is repeated until the desired accuracy is obtained. Note that after each guess for the pull-in voltage the switch must be allowed to open again. Again, $V_{\text {shift }}$ is determined by $V_{\text {shift }}=V_{\mathrm{pi}}-V_{\mathrm{pi},(t=0)}$.

3. Center shift method: Only measure the shift of the center (opened switch) part of the $C-V$ curve, and calculate the voltage at which the capacitance has the lowest value. This is done by fitting a parabola $C(V)=a \cdot(V-$ 


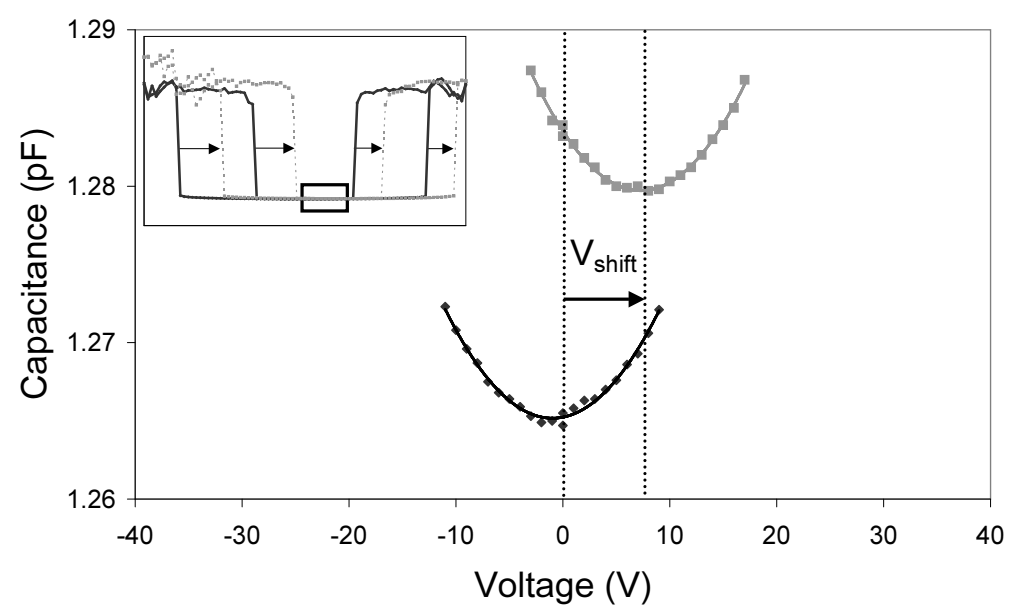

Figure 2.3: Center of the $C-V$ curve before (black) and after (grey) a switch has been stressed at 65 volt for 727 seconds. By fitting a parabola through the data, the center $V_{\text {shift }}$ can be accurately determined.

$\left.V_{\text {shift }}\right)^{2}+C_{\text {open }}$ through the center of the $C-V$ curve (Fig. 2.3). Since the electrostatic force is proportional to $\left(V-V_{\text {shift }}\right)^{2}[34], C(V)$ is symmetric around $V=V_{\text {shift }}$, therefore this fitted parabola accurately determines $V_{\text {shift }}$. To our knowledge, this is a new method. A known published non-contact method [27] differs from our method: the method described in [27] requires RF measurements and $V_{\text {shift }}$ is determined by manually tuning the bias voltage for minimum capacitance. The method proposed here is applicable for both low-frequency LCR meters and for RF equipment. It also requires no manual tuning of the bias voltage.

The whole $C$ - $V$ curve method has the disadvantage that for each measurement of $V_{\text {shift }}$, the capacitance has to be measured at least $4 \times V_{\text {pi }} / V_{\text {step }}$ times, where $V_{\text {step }}$ is the voltage step size with which the $C-V$ curve is measured. Since the LCR meter takes roughly 1 second to accurately measure the capacitance, measuring $V_{\text {shift }}$ takes a significant amount of time so that during the measurement of $V_{\text {shift }}$ the device may change (e.g. charge could leak away again). Also, the switch closes during the measurement of a whole $C-V$ curve so that the switch 'sees' additional stress during the determination of $V_{\text {shift }}$. The successive approximation method is faster and also more accurate, but still has one of the problems of the whole $C-V$ curve method: during the measurements voltages above $V_{\mathrm{pi}}$ have to be applied.

By contrast, the center shift method is faster and has no risk of charging the dielectric further during the determination of $V_{\text {shift }}$. In our case we measured the center part from the $C$ - $V$ curve in 17 steps, significantly less than the 162 steps 
required to measure a complete $C-V$ curve from -40 to $+40 \mathrm{~V}$ with $1 \mathrm{~V}$ resolution, and also less than the 26 steps used for the successive approximation method. Also, since a large $V_{\text {shift }}$ may prevent the switch from opening at $0 \mathrm{~V}$, the measurement voltage is first set to the previous value of $V_{\text {shift }}$, rather than $0 \mathrm{~V}$. Due to this step, the switch will be forced into the open branch of the hysteresis curve and open. The new value of $V_{\text {shift }}$ is determined by measuring $C(V)$ around the previous measured value of $V_{\text {shift }}$. This way, $V_{\text {shift }}$ can even still measured when $V_{\text {po }}^{-}>0$ (or $\left.V_{\mathrm{po}}^{+}<0\right)$.

To reduce the measurement time of $C-V$ curves, a second setup with which we can do reliability measurements was constructed. Here, instead of an off-theshelf LCR meter, a custom RF setup is used to measure the $C-V$ curve. The bias voltage is provided by an amplified signal from a function generator. Compared to the LCR setup this RF setup has several advantages as well as disadvantages: while the capacitance can be measured very quickly, it is more difficult to implement, requires some tedious calibration steps and requires the capacitive switches to have a layout which is compatible with RF-probes. It initially also had a much lower signal to noise ratio, but this has been successfully remedied by replacing the used 8-bit oscilloscope with a more accurate 12-bit data acquisition card (DAQ). It is discussed in more detail in section 2.3.

\subsubsection{Results: comparison of the measurement setups and methods}

In this paragraph we compare the influence of the different measurement methods and setups on the measurement results. In Fig. 2.4 and table 2.1 the effects and accuracy of the determination of $V_{\text {shift }}$ with the different methods and setups are shown. No stress voltage was applied between consecutive measurements of $V_{\text {shift }}$. Note that due to the intricacies of the individual measurement method, the way accuracy is determined varies between the methods. For the LCR successive approximation and LCR whole $C-V$ curve method, it is the resolution that follows from the minimum voltage step size, while for the other three methods it is the standard deviation in the measured $V_{\text {shift }}$ values.

As can be seen, the measured values of $V_{\text {shift }}$ obtained with the center shift method on the LCR setup only show a negligible drift (approximately $30 \mathrm{mV}$ at the $50^{t h}$ measurement). If we apply a linear fit to this data we find a drift rate of $0.3 \mathrm{mV}$ per measurement and an offset of $16 \mathrm{mV}$. The measured values of $V_{\text {shift }}$ obtained with the whole $C-V$ curve (also with the LCR setup) and successive approximation method show a significant change when measured repeatedly: with the successive approximation method was $-635 \mathrm{mV}$ after the $50^{\text {th }}$ measurement, more than 21 times larger than the drift of the center shift method. $V_{\text {shift }}$ is also measured very accurately with the center shift method: the standard deviation with respect to the linear fit is $8.2 \mathrm{mV}$. 


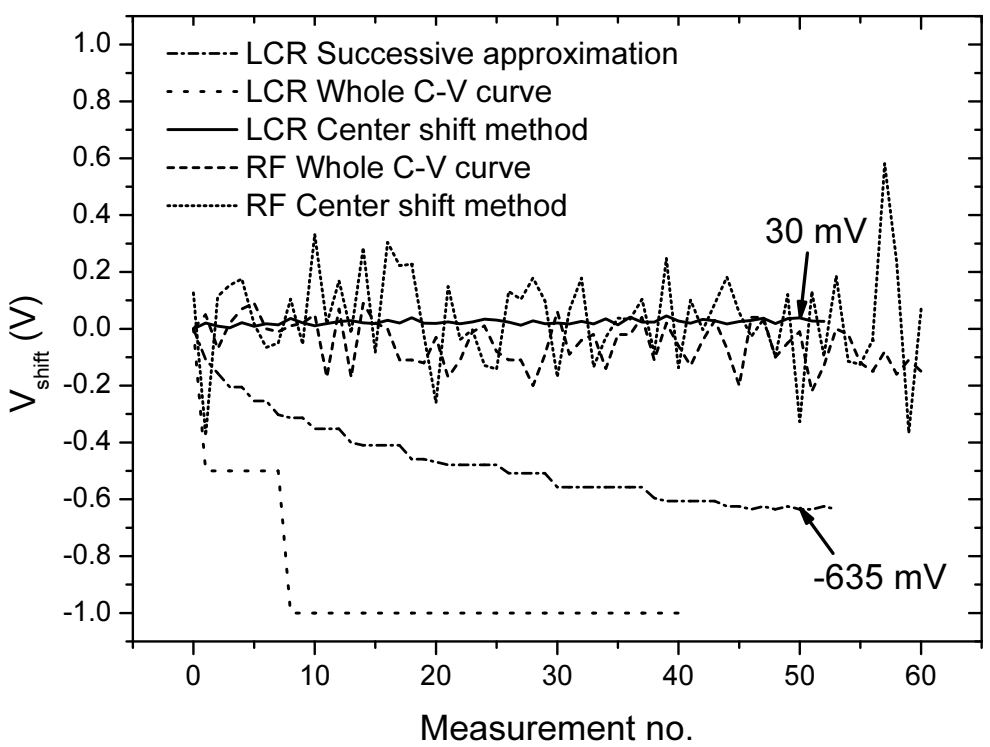

Figure 2.4: Effect of measurements on the voltage shift determined by measuring the change in pull-in voltage and by determining the center of the $C-V$ curve when applying no stress voltage. Before the switch was stressed, an initial $V_{\text {shift }}$ of $-444 \mathrm{mV}$ measured. This was subtracted from the center shift results for better comparison with the two other methods.

Table 2.1: Accuracy of the determination of $V_{\text {shift }}$, amount of time it takes to determine $V_{\text {shift }}$, and the effect the measurement has on the device for different methods.

\begin{tabular}{lllll}
\hline Measurement method & \# points & Time & Accuracy & $\begin{array}{l}\text { Drift after 20 } \\
\text { measurement } \\
\text { cycles }\end{array}$ \\
\hline $\begin{array}{l}\text { Whole } C \text { - } V \text { curve (LCR) } \\
\text { Successive approximation }\end{array}$ & 162 & $162 \mathrm{~s}$ & $0.5 \mathrm{~V}$ & $-1 \mathrm{~V}$ \\
of $V_{\mathrm{pi}}^{+}$(LCR) & 24 & $24 \mathrm{~s}$ & $50 \mathrm{mV}$ & $-0.47 \mathrm{~V}$ \\
Center shift (LCR) & 17 & $17 \mathrm{~s}$ & $<10 \mathrm{mV}$ & $<10 \mathrm{mV}$ \\
Whole $C$ - $V$ curve (RF) & 2000 & $0.4 \mathrm{~s}$ & $75 \mathrm{mV}$ & $<35 \mathrm{mV}$ \\
Center shift (RF) & 2000 & $0.1 \mathrm{~s}$ & $170 \mathrm{mV}$ & $<15 \mathrm{mV}$ \\
\hline
\end{tabular}


With the RF setup, there is no significant drift for both the center shift method and for the whole $C-V$ curve method. This shows that if the $C-V$ curve is measured fast enough (i.e. in $400 \mathrm{~ms}$ ), the whole $C-V$ curve method can also be used. However, due to the lower signal-to-noise ratio of the first version of the discrete $\mathrm{RF}$ setup and the noise on the amplified voltage from the signal generator, there is a larger spread in the measured voltage shift. The standard deviation on the values of $V_{\text {shift }}$ determined with the RF center shift method is $170 \mathrm{mV}$, which is mainly due to the uncertainty of the parabola fit. Although the accuracy of the parabola fit obtained with the RF setup is worse than the results obtained with the LCR setup ( $\sigma=170 \mathrm{mV}$ for the RF setup versus $\sigma=8.2 \mathrm{mV}$ for the LCR meter), the RF setup is faster (2.5 seconds versus 17 seconds for the whole $C-V$ curve). The standard deviation in the values of $V_{\text {shift }}$ determined from the change in $V_{\mathrm{pi}}$ is $80 \mathrm{mV}$.

All in all, the results clearly show the necessity of measuring charging effects in the open state by using the center shift method, or to very rapidly measure the $C$ - $V$ curve to avoid that the measurement itself influences the result by using a fast RF setup. They further show (as expected) that the LCR setup is slower and more accurate than the RF setup.

In Fig. 2.5 we have used the center shift method to determine the shifts in the $C-V$ curve due to three different stress voltages on 20 pristine devices. As one would expect, a higher stress voltage results in a faster and larger change of $V_{\text {shift }}$. According to Fig. 2.4, if these measurements had been done with the successive approximation method instead of the center shift method, a drift of about $-470 \mathrm{mV}$ would have been induced during the 20 measurements of $V_{\text {shift }}$ which were conducted during the 30 minutes of applied stress. This would give a significant deviation: after applying $50 \mathrm{~V}$ for 27 minutes the voltage shift of the $C-V$ curve ranged from $1.0 \mathrm{~V}$ to $1.8 \mathrm{~V}$, so that an error of $470 \mathrm{mV}$ would have been quite significant. Even at $60 \mathrm{~V}$, which resulted in shifts between $5.9 \mathrm{~V}$ and $6.6 \mathrm{~V}$, the drift part would have been $7.5 \%$ of the measured value. With the center shift method a drift of less than $6 \mathrm{mV}$ is expected. This indicates that the use of the center shift method is much better suited to characterize charging, especially at lower stress voltages and/or when $V_{\text {shift }}$ is measured relatively frequent. We also observe a spread in the measurement data. It is speculated that this is due to small variations in the composition, thickness of the dielectric, and surface roughness of the top electrode, which leads to variations in the electric field and charging current.

In Fig. 2.6 the RF setup is used to measure $V_{\text {shift }}$ (determined with the center shift method), and the positive and negative pull-in voltage extracted from the whole $C$ - $V$ curve as function of stress time at a stress voltage of $45 \mathrm{~V}$. Closer inspection reveals that the curves for $V_{\mathrm{pi}}^{+}$and $V_{\mathrm{pi}}^{-}$get closer together after more voltage stress has been applied, indicating a "narrowing" of the $C-V$ curve. To make this clearer, the voltage axis in Fig. 2.6 has been divided in three parts. Additionally, the $V_{\text {shift }}$ has also been determined as $\left(V_{\mathrm{pi}}^{+}+V_{\mathrm{pi}}^{-}\right) / 2$. As can be seen, 


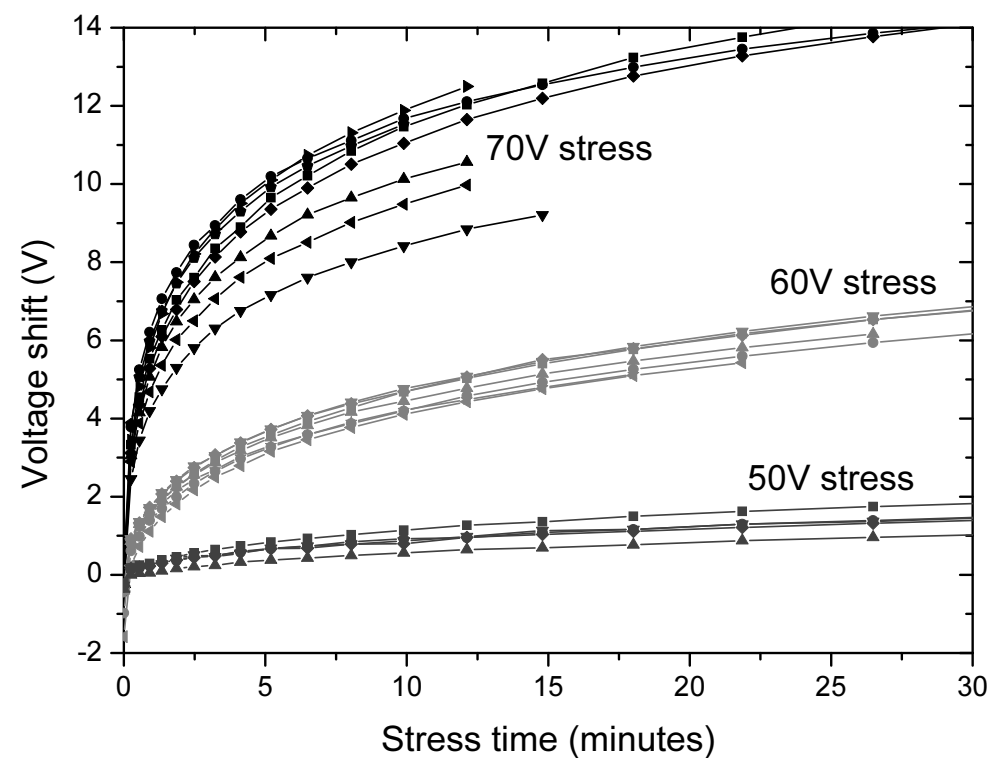

Figure 2.5: Shift of $C$ - $V$ curve as function of time at room temperature for 50, 60 and $70 \mathrm{~V}$ stress for 20 devices, measured with the center shift method on the LCR setup. Each device was not used prior to the stress test. 
the results almost overlap with the results from the fitted parabola.

The phenomenon of $C-V$ curve narrowing has been observed before and an explanation for this has been proposed by Rottenberg et al. [35]: if the injected charge is laterally inhomogeneous, the internal $E$-field caused by the charge can never be completely compensated by applying a laterally homogeneous $E$-field. The net result is that there are two effects: the center of the $C-V$ curve undergoes a shift proportional to the mean of the injected charge, while the lateral inhomogeneity results in an extra contribution in the electrostatic force which is proportional to the standard deviation of the amount of injected charge. This causes the $C-V$ curve to narrow, and the open state capacitance to increase.

Other causes for $C$ - $V$ curve narrowing could be changes in the spring constant and the gap height. $C$ - $V$ curve narrowing effects are discussed in more detail in chapter 3.2.1. Note that the narrowing effects are also visible in the $a$ and $C_{\text {open }}$ fit parameters of the parabola: the causes for narrowing the whole $C-V$ curve also increase the value of $a$ and $C_{\text {open }}$. However, we found that they are less useful for characterization than $V_{\text {shift }}, V_{\text {pi }}$, and $V_{\text {po }}$ as $a$ is sensitive to noise in the measured parabola, and $C_{\text {open }}$ to parasitic capacitances of the switch.

From the observed $C$ - $V$ curve narrowing we can conclude that even if the $C-V$ curve can be measured fast enough so that virtually no charge is injected during the $C$ - $V$ measurement, $V_{\text {shift }}$ should not be determined as a change in one of the pull-in voltages, but from the shift of the center of the $C-V$ curve. This center shift can be determined by fitting a parabola or from $\left(V_{\mathrm{pi}}^{+}+V_{\mathrm{pi}}^{-}\right) / 2$.

\subsubsection{Measurement methodology conclusions}

The effects of charge injection in the dielectric layer of an RF MEMS capacitive switch are studied using a center shift measurement method which is both accurate ( $8.2 \mathrm{mV}$ standard deviation on the LCR setup) and has negligible influence on the device under test, while the commonly used procedure of measuring the change in the pull-in voltage does have a significant influence on the device.

By measuring only the central part of the $C-V$ curve and fitting a parabola $C(V)=a \cdot\left(V-V_{\text {shift }}\right)^{2}+C_{\text {open }}$ to the data, $V_{\text {shift }}$ can be determined while the switch is in the open position, so that no additional charge is injected during the measurement, thereby separating the effect of intentionally induced stress and the stress effect that the measurement has on the device under test. This is especially important if dielectric charging at lower stress voltages is studied: at these lower voltages the $C-V$ curve shifts are smaller and slower, thereby requiring more accurate measurements. The method therefore meets the first of the conditions mentioned in section 2.1.

Another advantage is that if the $C$ - $V$ curve narrows due to laterally inhomogeneous charging (see section 3.2), a $V_{\text {shift }}$ determined with the center shift method will not be affected. This allows homogeneous charging to be studied indepen- 


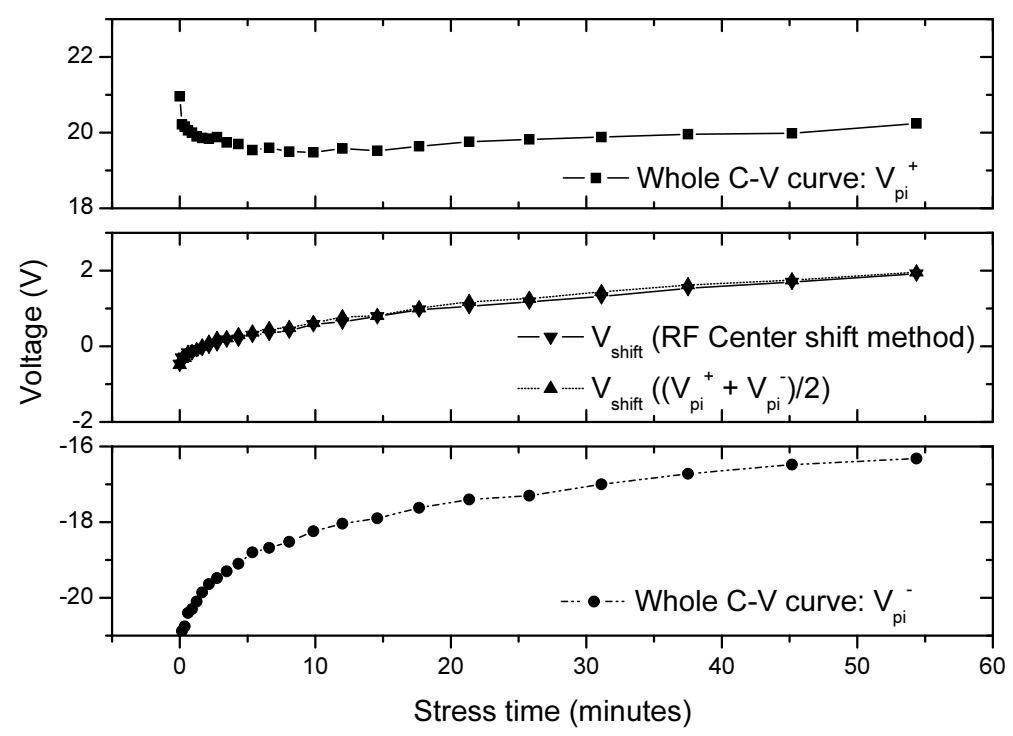

Figure 2.6: $V_{\mathrm{pi}}^{-}, V_{\text {shift }},\left(V_{\mathrm{pi}}^{+}+V_{\mathrm{pi}}^{-}\right) / 2$, and $V_{\mathrm{pi}}^{+}$as function of stress time as measured with the RF setup at a stress voltage of $45 \mathrm{~V}$.

dently from $C$ - $V$ curve narrowing effects. Because of this, the method also meets the second of the conditions mentioned in section 2.1. If experiments are done with $\mathrm{RF}$ equipment which quickly measure a $C$ - $V$ curve, the effect of the measurement on the device becomes negligible. In this case $V_{\text {shift }}$ may also be determined by $\left(V_{\mathrm{pi}}^{+}+V_{\mathrm{pi}}^{-}\right) / 2$.

\section{$2.3 \quad$ Fast RF 1-port S-parameter setup}

In chapter 2.2 we saw that dielectric charging can be studied by applying a stress voltage and periodically measuring (part of) the $C-V$ curve. From the data it was also apparent that an LCR-meter based setup can be too slow to correctly determine $V_{\text {shift }}$ if not used in the right way. Because of this, it was decided that a setup should be developed with which the $C$ - $V$ curve can be determined faster. One way to do this is is the method by Spengen et al. [36]. They constructed a reliability assessment setup that uses a $10.7 \mathrm{MHz}$ test signal in combination with a demodulator to determine changes in the capacitance. When compared to an LCR based setup, it has a much higher bandwidth. However, it is still a little bit too slow when measuring transient behavior, and it is challenging to accurately determine the exact capacitance. 
Another possibility is to use the method used by Nieminen et al. [37]. Based on their description, a custom single frequency 1-port S-parameter setup was made that can accurately measure transient behavior. It determines the capacitance by measuring the phase and amplitude of an RF signal reflected back at the probed MEMS switch. Using this method, the reflection can be determined with a sample rate of up to $60 \mathrm{MS} / \mathrm{s}$, much higher than the IF bandwidth of a Vector Network Analyzer, which is typically smaller than $100 \mathrm{kHz}$, or at most $5 \mathrm{MHz}$ for expensive high-end models.

To automate measurements software was written in the visual programming language National Instruments LabVIEW for acquisition and processing of measurement data. Since the software is able to generate a diverse array of stress patterns, and also controls a Microtech Cascade semi-automatic probe station, fully automated reliability measurements can be done. In the following paragraphs the hardware and software of the systems is discussed in more detail.

\subsubsection{Hardware}

The heart of the measurements system is a custom 1-port S-parameter setup. It is based on the description given by Nieminen et al. [37], and is schematically depicted in Fig. 2.7. It works as follows: an RF signal provided by a Rohde\&Schwarz SML03 RF Signal generator travels to a splitter (Mini-Circuits 15542 ZC3PD-900), which splits it in two parts. One part goes into the local oscillator of the IQ-demodulator, a ZAMIQ-895D made by Mini-Circuits. The other part passes through a circulator (Ditom D3C0890) to a bias-T (a combination of $100 \mathrm{pF}$ capacitor, $1 k \Omega$ resistor, and a small capacitor to ground), where a bias voltage can be added. The signal then reflects back from the RF MEMS capacitive switch. The reflected signal passes through the capacitor of the bias- $\mathrm{T}$, after which the circulator passes the signal to the RF-in port of the IQ-demodulator. The IQ-demodulator mixes the signal at the RF-in port with the LO port for the In-phase part (I) and also mixes it with a $90^{\circ}$ phase shifted LO for the Quadrature phase part (Q). The voltage at the I and Q port are measured using a NI PCI5105 data acquistion card. The 10-dB attenuator (Fig. 2.7) minimizes the effect of small non-linearities in the IQ-demodulator.

To calibrate the system, the remaining non-linearity of the IQ-demodulator must be measured to provide a lookup table for further reduction of the nonlinearity, and an open-short-load calibration must be performed to move the reference plane of the measurements to the probe tip. Only if both are done correctly, the capacitance can be calculated from the measured reflection. It is discussed in more detail in paragraph 2.3.2.

The bias voltage is provided by amplifying the signal from a Agilent 8657A function generator with a Krohn Hite 7602 Wideband Amplifier. The bias voltage is also measured at port 3 of the oscilloscope/DAQ. To measure a $C$ - $V$ curve, the function generator is programmed to produce a triangle wave. The magnitudes 


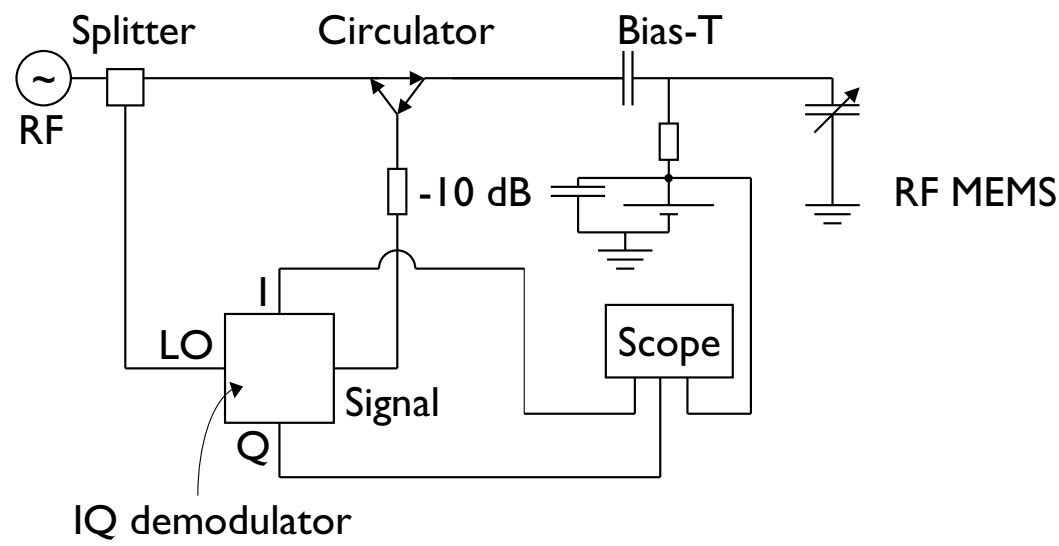

Figure 2.7: Schematic of the 1-port RF measurement system. Phase and amplitude of the reflected $\mathrm{RF}$ signal are measured with an IQ-demodulator, from which the capacitance can be determined.

of the up-ramp slope and down-ramp slope are equal. The trigger output of the signal generator should be connected with port 4 of the oscilloscope/DAQ.

Since the capacitance measurement speed is very high, the $C-V$ curve measurement time is limited by the response time of the MEMS and the bias-T. If the measurement is performed too quickly, the flanks at pull-in and pull-out in the $C-V$ curve are not vertical. If the fast bias-T consisting of a $100 \mathrm{pF}$ capacitor and $1 k \Omega$ resistor is used, the response time is limited by inertia and damping of the MEMS. If an additional $1 M \Omega$ resistor is placed in the bias path, the response time is dominated by the $R C$-time of the capacitance of the bias- $T$ and the resistor. The capacitive switch itself does not have significant effect on the $R C$-time, a typical capacitive switch has a much lower capacitance than the capacitor in the bias- $\mathrm{T}$.

To rule out these effects, the period of the triangle wave must be taken long enough. Generally a period of $400 \mathrm{~ms}$ is used, which is fast enough to have little effect on the device, but slow enough to rule out inertia effects. The bias voltage is also used to apply different types of stress to the device by programming the output pattern of the function generator.

A comparison of a $C-V$ curve measured with the LCR meter and with the custom 1-port S-parameter setup is shown in Fig. 2.8. It also shows the impact of sweep time on the measured $C-V$ curve: choosing a sweep time of $200 \mathrm{~ms}$ or below results in an overestimation of $V_{\mathrm{pi}}$. As can be seen, apart from small deviations in the closed state part of the LCR $C-V$ curve, and an offset in the open state capacitance, the two setups match quite well. 


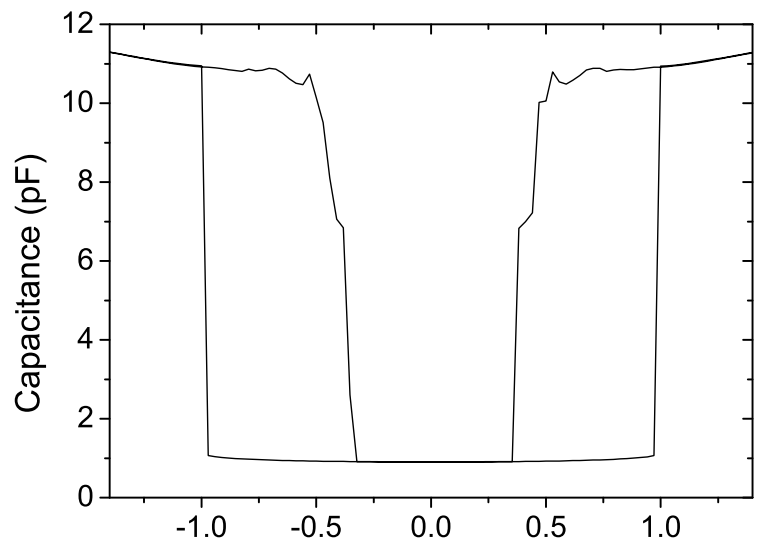

(a) Normalized voltage

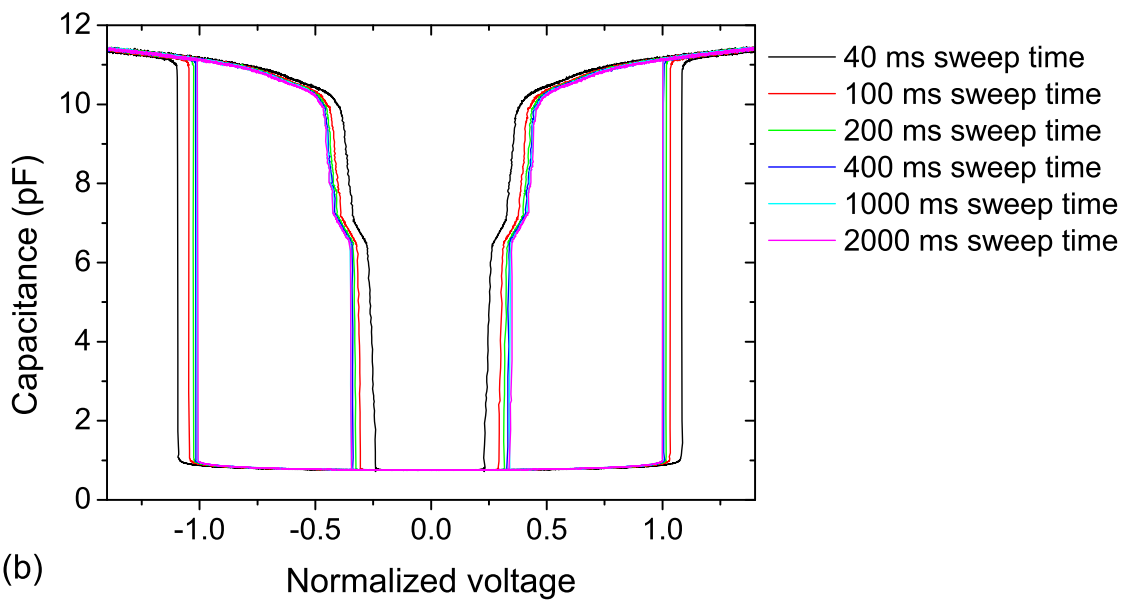

Figure 2.8: Comparison of the $C-V$ curve measured with an LCR meter (a), and using the custom 1-port S-parameter setup (b). (b) Also shows the effect of sweep time on the measured $C$ - $V$ curve. 


\subsubsection{Calibration of the measurement system}

Before the system can be used to measure capacitances, it must first be calibrated. A full calibration involves three steps:

1. Determining the exact gain of the high voltage amplifier.

2. Measuring the non-linear behavior of the IQ-demodulator to create a lookup table.

3. Doing an Open Short Load calibration to correct for cables and other passives.

Determining the gain of the $\mathrm{HV}$ amplifier is done in a very straightforward way: a number of voltages are applied by the function generator and the amplified voltage is measured with the oscilloscope/DAQ. A linear fit is used to determine gain and offset. Measuring and correcting for the non-linear behavior of the IQ-demodulator is not so trivial, and a correction method was developed which linearizes the measurement results by means of a lookup table. The open short load calibration method is quite common and also used in commercial network analyzers. The formulas for this were derived from the 2-port error box model found in application notes from Agilent [38].

The IQ-demodulator calibration requires a change in the cabling of the setup, which is shown in Fig. 2.9. RF1 is the Rohde\&Schwarz SML03 RF Signal generator connected to the Local Oscillator port of the IQ-demodulator and provides an 890 $\mathrm{MHz}$ signal at a constant level. RF2 is provided by a second signal generator, and is detuned $1 \mathrm{kHz}$ relative to $\mathrm{RF} 1$, so that at port $\mathrm{I}$ and $\mathrm{Q}$ there is a sine- and cosine-like signal. The $1 \mathrm{kHz}$ square wave provided by the function generator is for triggering purposes. A calibration lookup table is made by measuring the $\mathrm{I}$ and $\mathrm{Q}$ signal for different levels of the RF2 signal. Ideally this should lead to concentric circles when $\mathrm{Q}$ is plotted as function of $\mathrm{I}$. In reality the $\mathrm{I}$ and $\mathrm{Q}$ are not perfect sines and cosines, so that in an IQ-plot the measured circles are deformed. Also, the amplitude of the sine- and cosine-like I and Q signals are not a perfect linear function of the amplitude of the RF2 signal.

After the lookup table has been constructed from the (averaged) measurements, an algorithm $\mathfrak{M}$ is needed to map measured reflections to corrected reflections. First the mapping routine needs to determine where the reflection lies in the deformed spider weblike parameter space. It does this by comparing the amplitude of the measured reflection with the (interpolated) amplitude of the concentric rings of the calibration file at the angle of the measured reflection, moving from the center outwards.

Figures 2.10 and 2.11 illustrate how the measured reflection (small red dot) is mapped to the a corrected reflection (small black dot) using measured (large red dots) and corrected (large black dots) reflections in the lookup table. 


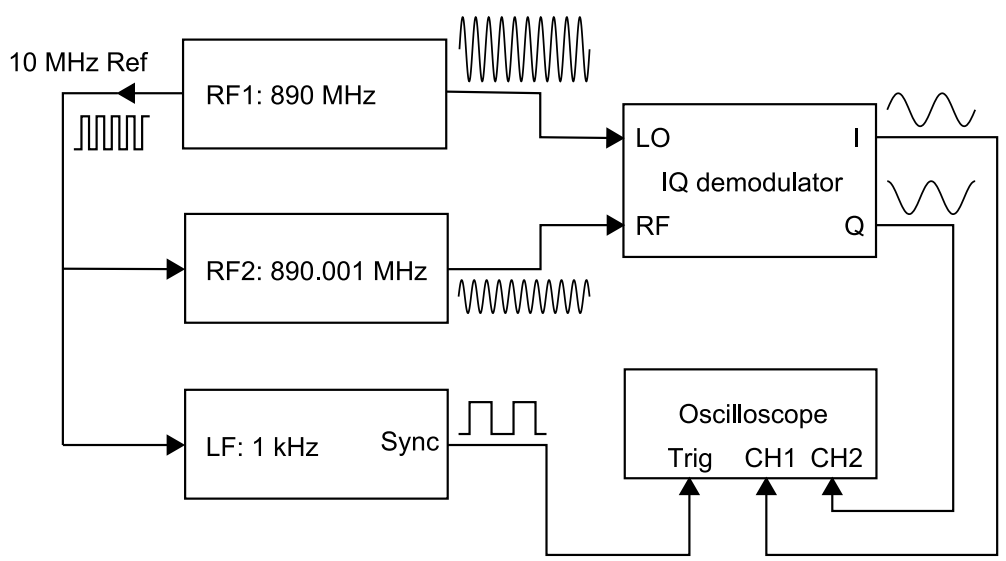

Figure 2.9: Schematic of the cabling for calibrating the IQ-demodulator.

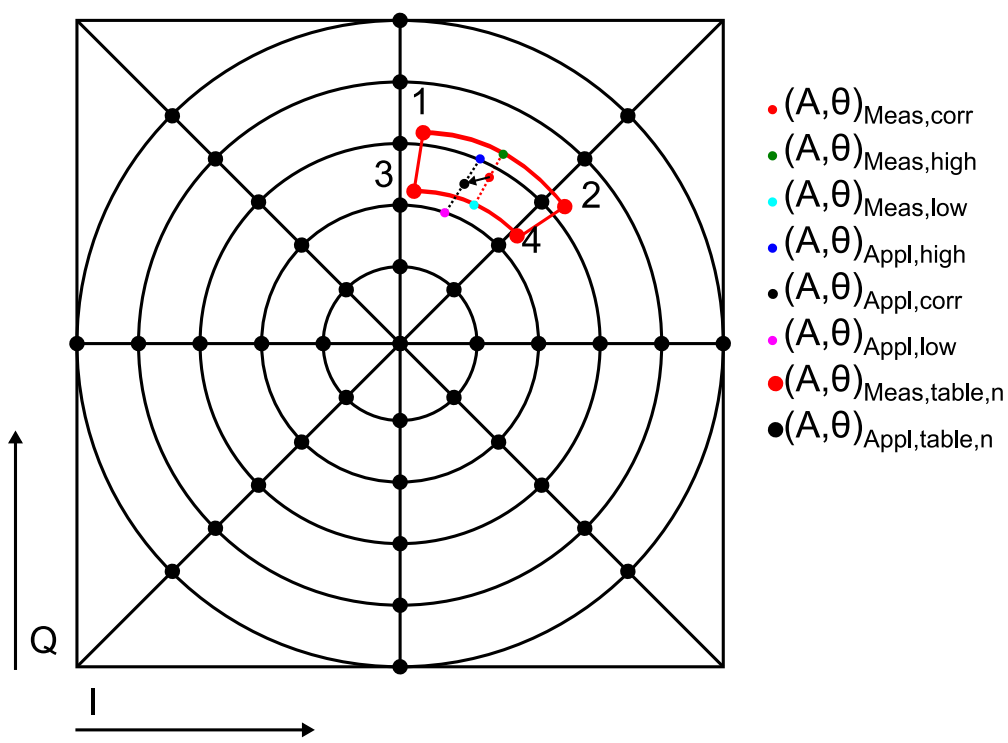

Figure 2.10: Schematic illustrating how measured reflections are corrected using the lookup table. Normally the actual used calibration map consists of more points. 


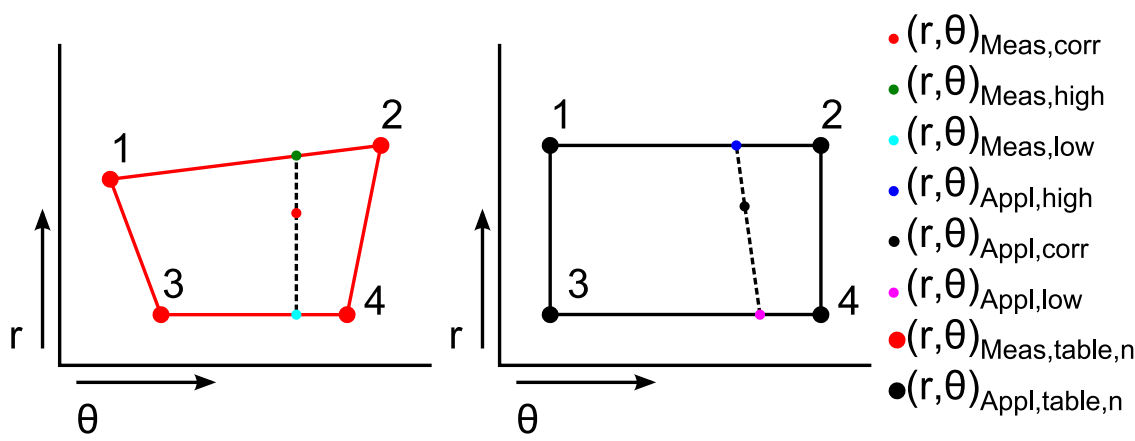

a)

b)

Figure 2.11: Zoomed in section of Fig. 2.10 detailing how the mapping algorithm corrects the deformed set of measured reflections back to the regularly space applied reflections. Polar coordinates $(r, \theta)$ are displayed in a cartesian way so that arcs become straight lines. a) Measured data. b) Corrected data.

When the four nearest reflection values $\left((r, \theta)_{\text {Meas,table, } \mathrm{n}}\right)$ in the calibration file are found with a search routine, first two variables, $(r, \theta)_{\text {Meas,low }}$ and $(r, \theta)_{\text {Meas, high }}$, are calculated. They are found by projecting the to be corrected reflection $(r, \theta)_{\text {Meas,corr }}$ onto the two arcs connecting the two lower and the two higher measured reflections (green and magenta dots in figures 2.10 and 2.11):

$$
\begin{aligned}
& r_{\text {Meas }, \text { high }}=r_{\text {Meas,table }, 1}+ \\
& \left(\frac{\theta_{\text {Meas,corr }}-\theta_{\text {Meas,table, } 1}}{\theta_{\text {Meas,table }, 2}-\theta_{\text {Meas,table }, 1}}\right) \cdot\left(r_{\text {Meas,table }, 2}-r_{\text {Meas,table, } 1}\right) \\
& r_{\text {Meas }, \text { low }}=r_{\text {Meas }, \text { table }, 3}+ \\
& \left(\frac{\theta_{\text {Meas,corr }}-\theta_{\text {Meas,table }, 3}}{\theta_{\text {Meas,table }, 4}-\theta_{\text {Meas,table }, 3}}\right) \cdot\left(r_{\text {Meas,table }, 4}-r_{\text {Meas,table }, 3}\right)
\end{aligned}
$$

Since the values $(r, \theta)_{\text {Meas,table,n }}$ were found during the calibration step, we have by definition:

$$
\mathfrak{M}\left((r, \theta)_{\text {Meas,table, } \mathrm{n}}\right)=(r, \theta)_{\mathrm{Appl}, \text { table, } \mathrm{n}}
$$

Since $r_{\text {Meas, high }}$ and $r_{\text {Meas,low }}$ were calculated from arcs connecting $(r, \theta)_{\text {Meas,table, } \mathrm{n}}$, we find that

$$
\begin{array}{r}
r_{\mathrm{Appl}, \mathrm{high}}=\mathfrak{M}\left(r_{\text {Meas }, \text { high }}\right)=r_{\mathrm{Appl}, \text { table }, 1}=r_{\mathrm{Appl}, \text { table }, 2} \\
r_{\mathrm{Appl}, \mathrm{low}}=\mathfrak{M}\left(r_{\text {Meas }, \text { low }}\right)=r_{\mathrm{Appl}, \text { table }, 3}=r_{\mathrm{Appl}, \text { table }, 4}
\end{array}
$$


The corrected amplitude can now be calculated by looking where point $(r, \theta)_{\text {Meas,corr }}$

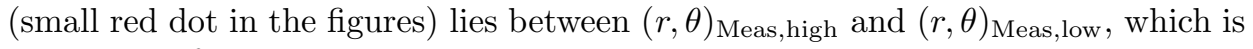
done in the following way:

$r_{\mathrm{Appl}, \mathrm{corr}}=r_{\mathrm{Appl}, \mathrm{low}}+\left(\frac{r_{\text {Meas }, \mathrm{corr}}-r_{\text {Meas }, \mathrm{low}}}{r_{\text {Meas,high }}-r_{\text {Meas }, \mathrm{low}}}\right) \cdot\left(r_{\mathrm{Appl}, \mathrm{high}}-r_{\mathrm{Appl}, \mathrm{low}}\right)$.

The phase $\theta$ is corrected in a similar way:

$\theta_{\mathrm{Appl}, \mathrm{corr}}=\theta_{\mathrm{Appl} \text { low }}+\left(\frac{r_{\text {Meas,corr }}-r_{\text {Meas,low }}}{r_{\text {Meas,high }}-r_{\text {Meas,low }}}\right) \cdot\left(\theta_{\mathrm{Appl}, \text { high }}-\theta_{\mathrm{Appl}, \mathrm{low}}\right)$.

Here $\theta_{\mathrm{Appl} \text {,high }}$ and $\theta_{\mathrm{Appl}, \text { low }}$ are found by looking where they lie on the arcs between the points $(r, \theta)_{\mathrm{Appl}, \text { table,n }}$ :

$$
\begin{aligned}
& \theta_{\mathrm{Appl}, \text { high }}=\theta_{\mathrm{Appl}, \text { table }, 1}+ \\
& \left(\frac{\theta_{\text {Meas }, \text { corr }}-\theta_{\text {Meas,table }, 1}}{\theta_{\text {Meas,table }, 2}-\theta_{\text {Meas,table }, 1}}\right) \cdot\left(\theta_{\text {Appl,table }, 2}-\theta_{\text {Appl,table }, 1}\right) \\
& \theta_{\mathrm{Appl}, \mathrm{low}}=\theta_{\mathrm{Appl}, \mathrm{table}, 3}+ \\
& \left(\frac{\theta_{\text {Meas,corr }}-\theta_{\text {Meas,table }, 3}}{\theta_{\text {Meas,table } 4}-\theta_{\text {Meas }, \text { table }, 3}}\right) \cdot\left(\theta_{\mathrm{Appl}, \text { table }, 4}-\theta_{\mathrm{Appl}, \text { table }, 3}\right) .
\end{aligned}
$$

The correction method described above reduces the nonlinearity from by typically a factor 5 to 10 . This is shown in Fig. 2.12, where the relative error in the measured amplitude of an RF signal (a pure sine function) is plotted as function of phase difference with the LO before and after correction. Before correction of nonlinearity, the measured amplitude varies with applied phase. For low applied signals this is a deviation of up to $3 \%$, for higher amplitudes this can be up to $10 \%$. After correction the deviation is greatly reduced. If measured immediately after the calibration, deviations are reduced to only $0.25 \%$. After a some time (a few hours) the system will exhibit drift and the phase-averaged mean difference between applied and measured amplitude is reduced from $1-5 \%$ to $1 \%$. The phase dependence of the measured amplitude is reduced even more: from uncalibrated $3-10 \%$ to typically $0.05-0.75 \%$ (typically $0.1 \%$ ). As already noted, the quality of the correction depends on the measurement conditions and the time between calibration and measurement. However, other measurements have shown that using a several months old calibration file is not significantly worse than using a several hours old calibration file.

After the non-linearity has been corrected for, phase and magnitude of a reflected signal can be accurately measured. However, since the setup is a 1-port single frequency vector network analyzer, the internal reflections and losses in cables, the RF probe, and other passive components must be known in order to 

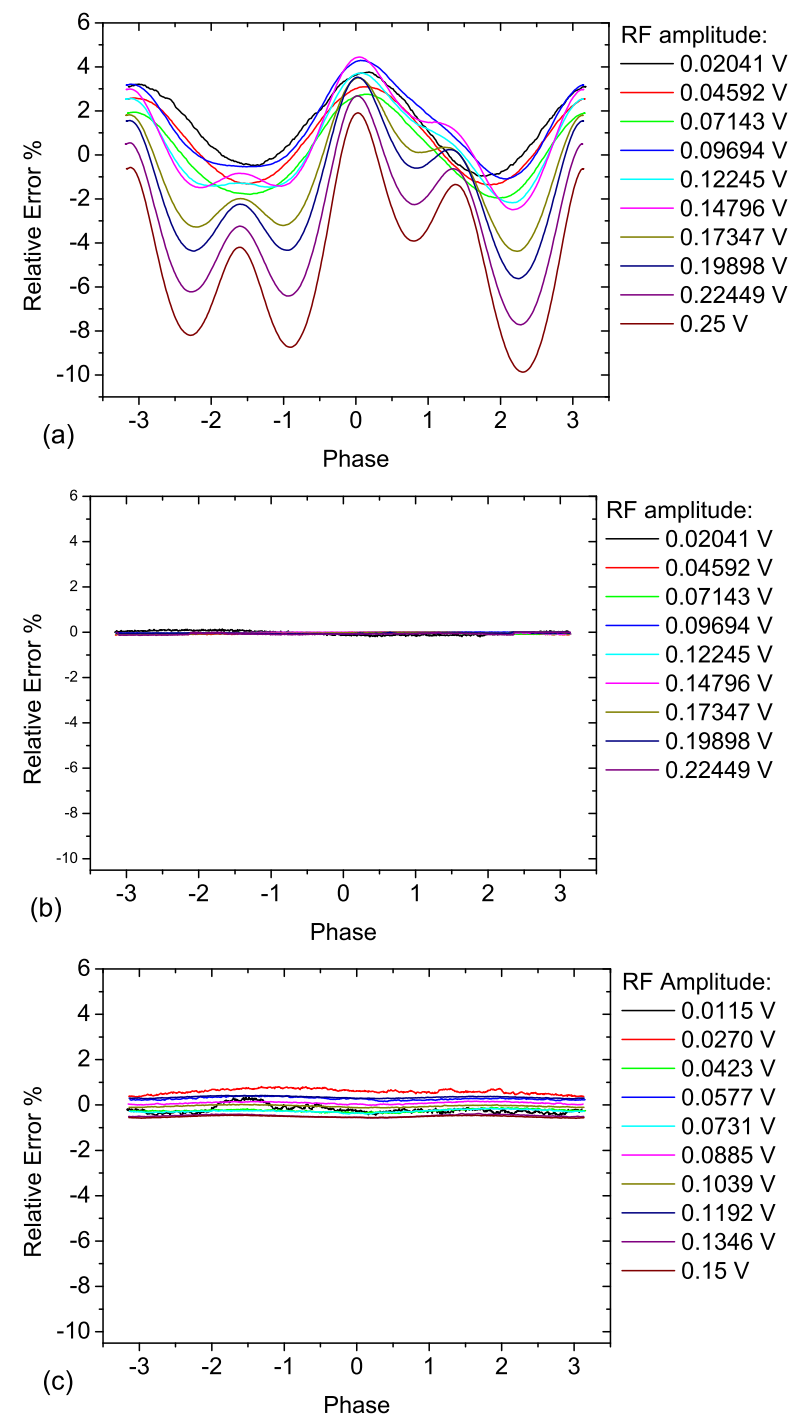

Figure 2.12: Error in the measured amplitude of an RF signal (a pure sine function) plotted as function of phase difference with the LO before and after correction for nonlinearities. (a) Before correction. (b) Correction immediately after making the calibration file. (c) Correction after several hours. 


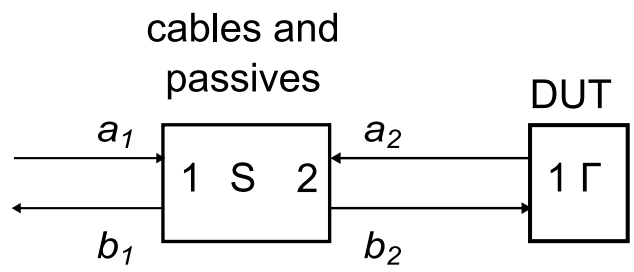

Figure 2.13: Schematic showing how phase shifts and losses due to cables, RF probe, and other passive components can be modelled.

calculate the reflection coefficient of a device under test. This can be described as a 2-port error box [38]. See also Fig. 2.13. In the figure $a_{1}$ is the incoming wave at port 1 (coming from the RF signal generator), $a_{2}$ the incoming wave at port 2 , $b_{1}$ the wave travelling away from port 1 (detected by IQ demodulator), and $b_{2}$ the wave travelling away from port 2 . The relation between incoming and outgoing waves can be described as:

$$
\left[\begin{array}{l}
b_{1} \\
b_{2}
\end{array}\right]=\mathbf{S}\left[\begin{array}{l}
a_{1} \\
a_{2}
\end{array}\right] .
$$

When the DUT has a reflection coefficient $\Gamma_{\mathrm{A}}$, we get the following equations for the outgoing wave at port 1:

$$
\begin{aligned}
& b_{1}=S_{11} a_{1}+S_{21} a_{2} \\
& a_{2}=\Gamma_{\mathrm{A}} b_{2} \\
& b_{2}=S_{12} a_{1}+S_{22} a_{2} .
\end{aligned}
$$

Eliminating $a_{2}$ and $b_{2}$ we get

$$
\Gamma_{\mathrm{M}}=\frac{b_{1}}{a_{1}}=S_{11}+\frac{\Gamma_{\mathrm{A}} S_{12} S_{21}}{1-\Gamma_{\mathrm{A}} S_{22}}
$$

From this we see that when we measure $\Gamma_{\mathrm{M}}, \Gamma_{\mathrm{A}}$ of the DUT can be determined when we know $S_{11}, S_{22}$ and the product $S_{12} S_{21}$ (we do not need to know $S_{12}$ and $S_{21}$ separately). To determine these three unknowns, $b_{1} / a_{1}$ must be measured for three different $\Gamma$ 's (an open, short and load $(50 \Omega)$ standard on a calibration substrate), so that we get three equations with three unknowns. The three solutions 
are then given by:

$$
\begin{aligned}
S_{11} & =\frac{\Gamma_{\mathrm{A} 2} \Gamma_{\mathrm{A} 3} \Gamma_{\mathrm{M} 1} \Delta_{\mathrm{M} 23}+\Gamma_{\mathrm{A} 1}\left(\Gamma_{\mathrm{A} 2} \Gamma_{\mathrm{M} 3} \Delta_{\mathrm{M} 12}+\Gamma_{\mathrm{A} 3} \Gamma_{\mathrm{M} 2} \Delta_{\mathrm{M} 31}\right)}{\Gamma_{\mathrm{A} 2} \Gamma_{\mathrm{A} 3} \Delta_{\mathrm{M} 23}+\Gamma_{\mathrm{A} 1}\left(\Gamma_{\mathrm{A} 2} \Delta_{\mathrm{M} 12}+\Gamma_{\mathrm{A} 3} \Delta_{\mathrm{M} 31}\right)} \\
S_{22} & =\frac{\Gamma_{\mathrm{A} 3} \Delta_{\mathrm{M} 21}+\Gamma_{\mathrm{A} 2} \Delta_{\mathrm{M} 13}+\Gamma_{\mathrm{A} 1} \Delta_{\mathrm{M} 32}}{\Gamma_{\mathrm{A} 2} \Gamma_{\mathrm{A} 3} \Delta_{\mathrm{M} 23}+\Gamma_{\mathrm{A} 1}\left(\Gamma_{\mathrm{A} 2} \Delta_{\mathrm{M} 12}+\Gamma_{\mathrm{A} 3} \Delta_{\mathrm{M} 31}\right)} \\
S_{12} S_{21} & =\frac{\Delta_{\mathrm{A} 12} \Delta_{\mathrm{A} 13} \Delta_{\mathrm{A} 23} \Delta_{\mathrm{M} 12} \Delta_{\mathrm{M} 31} \Delta_{\mathrm{M} 23}}{\left(\Gamma_{\mathrm{A} 2} \Gamma_{\mathrm{A} 3} \Delta_{\mathrm{M} 23}+\Gamma_{\mathrm{A} 1}\left(\Gamma_{\mathrm{A} 2} \Delta_{\mathrm{M} 12}+\Gamma_{\mathrm{A} 3} \Delta_{\mathrm{M} 31}\right)\right)^{2}} .
\end{aligned}
$$

Here $\Gamma_{\mathrm{Ai}}$ are the three reflection coefficients of the calibration substrate and $\Gamma_{\mathrm{Mi}}$ the measured reflections. $\Delta_{\mathrm{Aij}}$ is defined as $\Gamma_{\mathrm{Ai}}-\Gamma_{\mathrm{Aj}}$, and $\Delta_{\mathrm{Mij}}$ as $\Gamma_{\mathrm{Mi}}-\Gamma_{\mathrm{Mj}}$. After $S_{11}, S_{22}$, and $S_{12} S_{21}$ have been determined, they can be used to find $\Gamma_{\mathrm{A}}$ of an actual switch by inverting Eq. 2.14:

$$
\Gamma_{\mathrm{A}}=\frac{\Gamma_{\mathrm{M}}-S_{11}}{\left(\Gamma_{\mathrm{M}}-S_{11}\right) S_{22}+S_{12} S_{21}} .
$$

From this the impedance can be calculated [39] using:

$$
Z=50 \frac{\Gamma+1}{\Gamma-1} .
$$

\subsubsection{Measurement automation}

In order to reliably obtain a large amount of measurement data, all necessary measurement types were automated using the visual programming language NI LabVIEW. This involves programs for doing the calibration (described in 2.3.2), stress-tests, measuring $C$ - $V$ curves, closing and opening transients. All except the calibration routines can be used in combination with a Cascade Microtech Summit $12 \mathrm{~K}$ semi-automatic probe station.

Measuring a $C-V$ curve is then straightforward: program the signal generator to generate one or more periods of a triangle wave and measure the voltage at the I and Q port of the IQ-demodulator. After this, the results are corrected using the methods described in paragraph 2.3.2. Measuring transients is done in a similar way, but instead of using a (relatively slow) triangle wave, block-shaped pulses are used. For the stress a DC voltage, square wave, or custom arbitrary waveforms can be applied

The stress-test program is a bit more complex, and involves the following steps:

1. Measure full $C-V$ curve and save it.

2. Extract $V_{\mathrm{pi}}^{ \pm}$and $V_{\text {po }}^{ \pm}$from the data.

3. Measure a $C-V$ parabola and save it to disk. 
4. Extract $V_{\text {shift }}$ from the parabola.

5. Determine if the program needs to stop, apply stress, or let the device recover.

6. Apply stress, let the device recover or stop.

7. Set the voltage to the previously measured $V_{\text {shift }}$ to let the switch open

8. Repeat from 1.

Since in general the switch degrades much faster in the beginning of a stress test, the stress-time between two $C-V$ curve measurements is increased exponentially until it reaches a set maximum. Also, $V_{\mathrm{pi}}$ and $V_{\text {po }}$ are automatically detected by an extraction routine that searches for maxima and minima of the derivative of the $C-V$ curve. Note that a parabola is also measured even though the whole $C-V$ curve is measured. This is done because even though in principle $V_{\text {shift }}$ is equal to $\left(V_{\mathrm{pi}}^{+}+V_{\mathrm{pi}}^{-}\right) / 2$, in practice a small difference is sometimes observed when the switch undergoes a slight mechanical deformation due to the measurement of the whole $C-V$ curve.

Since the stress tests sometimes require a lot of time, it is useful if the program can stop after a certain amount of degradation. Therefore the program was written so that it can stop on the following criteria:

- The amount of stress time has passed a preset limit.

- The absolute value of $V_{\text {shift }}$ has become greater than a limit value.

- The $V_{\mathrm{pi}}^{+}-V_{\mathrm{pi}}^{-}$has decreased by a certain percentage, or has increased by more than 10 percent (the latter indicating a failure of the pull-in voltage extraction routine since in general a $C-V$ curve does not become wider under stress).

After the program has finished, relevant data such as $V_{\mathrm{pi}}^{ \pm}, V_{\mathrm{po}}^{ \pm}, V_{\text {shift }}, C_{\text {open }}$, and $C_{\text {close }}$ are saved as function of stress time.

\subsection{Conclusions}

Studying the reliability of RF MEMS capacitive switches requires careful consideration of the used measurement methodology and measurement system. The effect of the measurement on the device must be negligible compared to the effect of the stress itself, and the change in the measured parameter(s) must be related to only one underlying degradation mechanism, or be disentangled into the individual rootcauses. When characterizing homogeneous dielectric charging, the newly proposed parabola method for measuring $V_{\text {shift }}$ satisfies these criteria. Furthermore, a fast 
RF 1-port S-parameter measurement system was developed which makes it possible to further minimize the effect of the measurement on the device, and provides a flexible platform for characterizing reliability of capacitive switches. By using a table-based method to correct for nonlinearities in the used IQ-demodulator, and Open Short Load calibration to correct for cables and other passive components, the impedance of a switch is determined both quickly (sub-microsecond time resolution, much faster than a typical commercial off-the-shelf vector network analyzer), and accurately (up to femto farad range for capacitors). 


\section{Chapter 3}

\section{Degradation mechanisms in capacitive switches}

\subsection{Introduction}

In chapter 2 the requirements on measurement methodology and measurement system with respect to the study of the reliability of RF MEMS capacitive switches were discussed. We showed how the parabola method, which was introduced to minimize the effects of the measurement on the device, could be used to characterize dielectric charging. In this chapter we demonstrate how, in addition to characterizing homogeneous charging with the parabola method, two other degradation mechanisms, namely laterally inhomogeneous charging and mechanical degradation, can be identified from changes of the $C-V$ curve as a whole. This is then used in experiments that each target one specific degradation mechanism in order to gain more insight in how these different mechanisms work. Additionally, we will study laterally inhomogeneous charging in more detail using Scanning Kelvin Probe Microscopy.

\subsection{Identification of degradation mechanisms}

In chapter 1.2 we mentioned that many papers on the degradation of RF MEMS capacitive switches focus on dielectric charging [18-26]. The main effect of dielectric charging is that it results in a built-in voltage that shifts the entire $C$ - $V$ curve $[21,24,27,28]$. An additional, but a less documented observation is that the positive and negative pull-in voltage move closer to each other $[23,40]$. An explanation for this narrowing of the $C-V$ curve was proposed by Rottenberg et al. [35] based on the assumption that the charge is injected inhomogeneously. Later publications 

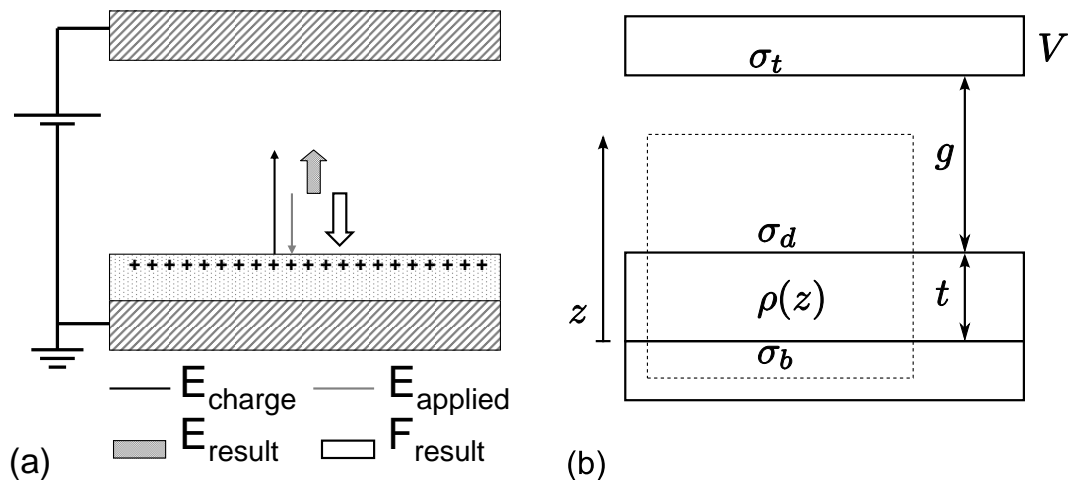

Figure 3.1: (a) Schematic representation of how trapped charge change the electric field and electrostatic force in a capacitive switch. (b) Position of (surface) charge densities and Gauss box in the capacitive switch.

of the same group [41-44] reported more about the conditions under which the $C-V$ curve will narrow due to inhomogeneous charge injection. However, there is a second possible explanation for the narrowing effect: a reduction of the gap due to mechanical degradation of the springs will also narrow the $C-V$ curve $[44,45]$.

To distinguish which of these two degradation mechanisms causes the narrowing effects when a switch is stressed, we will first show that, given a certain amount of change in the pull-in voltage, the amount of change in the pull-out voltage will depend on the degradation mechanism. After this we present measurements which show that both mechanical deformation and inhomogeneous dielectric charging can occur, depending on the type of stress that is applied. This also shows that beside uniform dielectric charging, inhomogeneous charging and mechanical degradation can also be investigated by applying criteria $2 \mathrm{~b}$ and $2 \mathrm{c}$ from section 2.1 .

\subsection{1 $C-V$ curve narrowing theory}

MEMS reliability studies have shown [18-26] that due to the high electric fields charge can be injected in the dielectric. The trapped charges change the electric field (Fig. 3.1a) and lead to a built-in voltage $V_{\text {shift }}$ which shifts the $C$ - $V$ curve $[21,24,27,28]$. This $V_{\text {shift }}$ is proportional to the amount of charge, and to the distance between the trapped charge and the bottom electrode.

To see how this works, we consider the situation of a charged MEMS switch (Fig. 3.1b) with a height dependent charge density $\rho(z)$ trapped in the dielectric, a surface charge density $\sigma_{d}$ on top of the dielectric, and two surface charge densities $\sigma_{b}$ and $\sigma_{t}$ in the bottom and top electrode to maintain charge neutrality. The dielectric has a thickness $t$ and there is a gap with height $g$, where $g$ is equal to 
$g_{0}+u$ (the top electrode does not have to be in the neutral position). $z=0$ lies at the interface between bottom electrode and the dielectric. A voltage $V$ is applied to the top electrode. By definition, $V$ is equal to

$$
V=-\int_{0}^{t+g} E(z) \mathrm{d} z
$$

With Gauss' law we find that $E(z)$ is equal to

$$
E(z)=\left\{\begin{array}{cl}
\frac{\sigma_{b}+\int_{0}^{z} \rho\left(z^{\prime}\right) \mathrm{d} z^{\prime}}{\epsilon_{0} \epsilon_{r}} & (0<z<t) \\
\frac{\sigma_{b}+\int_{0}^{t} \rho\left(z^{\prime}\right) \mathrm{d} z^{\prime}+\sigma_{d}}{\epsilon_{0}} & (t<z<g+t)
\end{array}\right.
$$

Here the Gauss box was chosen with one side in the bottom electrode, and the other side at height $z$. If we choose the box with one side in the top electrode, we find that

$$
E(z)=-\frac{\sigma_{t}}{\epsilon_{0}} \quad(t<z<g+t) .
$$

Applying Gauss' law to the complete structure (one side of the Gauss box in the top electrode, the other side in the bottom electrode), we find that the structure must be electrically neutral:

$$
\sigma_{b}+\int_{0}^{t} \rho(z) \mathrm{d} z+\sigma_{d}+\sigma_{t}=0
$$

Note that this also follows from Eq. 3.2 and 3.3. Filling Eq. 3.2 into Eq. 3.1 results in

$$
V=-\frac{\sigma_{b}}{\epsilon_{0}}\left(\frac{t}{\epsilon_{r}}+g\right)-\frac{1}{\epsilon_{0}}\left(\frac{\int_{0}^{t} \int_{0}^{z} \rho\left(z^{\prime}\right) \mathrm{d} z^{\prime} \mathrm{d} z}{\epsilon_{r}}+g \int_{0}^{t} \rho\left(z^{\prime}\right) \mathrm{d} z^{\prime}\right)-\frac{g \sigma_{d}}{\epsilon_{0}} .
$$

By using integration by parts we find that

$$
\int_{0}^{t} \int_{0}^{z} \rho\left(z^{\prime}\right) \mathrm{d} z^{\prime} \mathrm{d} z=\left[z \int_{0}^{z} \rho\left(z^{\prime}\right) \mathrm{d} z^{\prime}\right]_{0}^{t}-\int_{0}^{t} z \rho(z) \mathrm{d} z .
$$

Combining this with Eq. 3.5 gives

$$
V=-\frac{\sigma_{b}}{\epsilon_{0}}\left(\frac{t}{\epsilon_{r}}+g\right)-\frac{1}{\epsilon_{0}} \int_{0}^{t}\left(\frac{t}{\epsilon_{r}}+g-\frac{z}{\epsilon_{r}}\right) \rho(z) \mathrm{d} z-\frac{g \sigma_{d}}{\epsilon_{0}} .
$$


Since the electrostatic force for a parallel plate capacitor is equal to $-A(E)(g+$ $t))^{2} / 2 \epsilon_{0}$ [46], we want to know how the $E(g+t)$ changes due to the trapped charge. We can find $E(g+t)$ by eliminating $\sigma_{b}$ from equations 3.2 and 3.7 :

$$
E(g+t)=\frac{1}{t / \epsilon_{r}+g}\left(-V+\frac{1}{\epsilon_{0} \epsilon_{r}} \int_{0}^{t} z \rho(z) \mathrm{d} z+\frac{t \sigma_{d}}{\epsilon_{0} \epsilon_{r}}\right)=-\frac{V-V_{\text {shift }}}{t / \epsilon_{r}+g},
$$

where $V_{\text {shift }}$ is equal to

$$
V_{\text {shift }}=\frac{1}{\epsilon_{0} \epsilon_{r}}\left[\int_{0}^{t} \rho(z) \mathrm{d} z+t \sigma_{d}\right]
$$

We thus find that $V_{\text {shift }}$ is proportional to the amount of trapped charge, as well as the distance of the charge from the bottom electrode. Positive charge will shift the $C$ - $V$ curve in the positive direction, negative charge will shift it in the negative direction.

However, while a built-in voltage explains the shifting of a $C-V$ curve, it does not explain the narrowing observed in [23,40-43]. Three causes can account for this narrowing effect:

1 A laterally inhomogeneous distribution of the trapped charge [35,41-44]. (See explanation below)

2 A reduced open state gap $g_{0}$ due to mechanical deformation. (Eq. 1.9 and Eq. 1.10)

3 A reduction of the spring constant $k$. (Eq. 1.9 and Eq. 1.10)

Of these three causes, the third one is not very likely, as the spring constant is mainly a function of the size and geometry of the springs and its material properties, which both do not change significantly during stress tests. The first cause is explained below and in $[35,44]$, the second and third causes can be understood from Eq. 1.9 and Eq. 1.10.

When charge is injected inhomogeneously, the expression for the electrostatic force changes from Eq. 1.4 to [35]:

$$
\begin{aligned}
F_{E} & =-\frac{\epsilon_{0}}{2} \int_{\text {Area }} \frac{\left(V-V_{\text {shift }}(x, y)\right)^{2}}{\left(t / \epsilon_{r}+g\right)^{2}} \mathrm{~d} A \\
& =-\frac{\epsilon_{0} A}{2} \frac{\left(V-\bar{V}_{\text {shift }}\right)^{2}+\sigma_{V_{\text {shift }}}^{2}}{\left(t / \epsilon_{r}+g\right)^{2}}
\end{aligned}
$$


where $V_{\text {shift }}(x, y)$ is the position dependent built-in voltage, $\bar{V}_{\text {shift }}$ the average of $V_{\text {shift }}(x, y)$, and $\sigma_{V_{\text {shift }}}$ the standard deviation of $V_{\text {shift }}(x, y)$. This equation assumes that the characteristic length scale over which the charge density varies in the lateral direction is large compared to $g_{0}$, so that the parallel plate equation is still valid. From the equation we see that by applying a voltage we can only compensate for the contribution to $F_{E}$ of $\bar{V}_{\text {shift }}$, and always have the additional force due to $\sigma_{V_{\text {shift }}}$. When comparing this with Eq. 1.4 we see that the $g$-dependence has not changed, and if the open state gap has not changed, the force balance remains essentially the same. In the inhomogeneously charged situation pull-in will occur when the value of the nominator is equal to the value it has for the uncharged situation at pull-in:

$$
\begin{array}{r}
\left(V_{\mathrm{pi}}-\bar{V}_{\text {shift }}\right)^{2}+\sigma_{V_{\text {shift }}}^{2}=V_{\mathrm{pi},(t=0)}^{2} \Longrightarrow \\
V_{\mathrm{pi}}^{ \pm}=\bar{V}_{\text {shift }}+V_{\mathrm{pi},(t=0)}^{ \pm} \sqrt{1-\sigma_{V_{\text {shift }}^{2}}^{2} / V_{\mathrm{pi},(t=0)}^{2}},
\end{array}
$$

where $V_{\mathrm{pi},(t=0)}^{ \pm}$is the pull-in voltage of the unstressed device. A similar argument can also be made for the pull-out voltage, where we find that

$$
\begin{aligned}
\left(V_{\mathrm{po}}-\bar{V}_{\text {shift }}\right)^{2}+\sigma_{V_{\text {shift }}}^{2}=V_{\mathrm{po},(t=0)}^{2} \Longrightarrow \\
V_{\mathrm{po}}^{ \pm}=\bar{V}_{\text {shift }}+V_{\mathrm{po},(t=0)}^{ \pm} \sqrt{1-\sigma_{V_{\text {shift }}}^{2} / V_{\mathrm{po},(t=0)}^{2}},
\end{aligned}
$$

Here $V_{\mathrm{po},(t=0)}^{ \pm}$is the pull-out voltage of the unstressed device. To separate homogeneous charging effects from inhomogeneous charging effects, we here introduce the entities $\left|V_{\text {pi }}\right|$ and $\left|V_{\text {po }}\right|$, which we define as

$$
\begin{aligned}
& \left|V_{\mathrm{pi}}\right|=\left(V_{\mathrm{pi}}^{+}-V_{\mathrm{pi}}^{-}\right) / 2 \\
& \left|V_{\mathrm{po}}\right|=\left(V_{\mathrm{po}}^{+}-V_{\mathrm{pi}}^{-}\right) / 2
\end{aligned}
$$

Since by definition $\left|V_{\mathrm{po}}\right|$ is smaller than $\left|V_{\mathrm{pi}}\right|$, inhomogeneous charging will cause a larger change in $\left|V_{\mathrm{po}}\right|$ than in $\left|V_{\mathrm{pi}}\right|$. Also, Eq. 3.12 shows that when $\sigma_{V_{\text {shift }}}$ is equal to $V_{\mathrm{po},(t=0)}$, the switch will not open anymore.

When the open state gap $g_{0}$ is reduced by mechanical degradation of the springs, but no inhomogeneous charge is injected, the change in $V_{\text {pi }}$ can be found from the 1-D solutions for pull-in (Eq. 1.9):

$$
\begin{array}{r}
V_{\mathrm{pi}}^{ \pm}= \pm \sqrt{\frac{8 k\left(t / \epsilon_{r}+g_{0}\right)^{3}}{27 \epsilon_{0} A} \Longrightarrow} \\
V_{\mathrm{pi}}^{ \pm}=V_{\mathrm{pi},(t=0)}^{ \pm} \sqrt{\frac{\left(t / \epsilon_{r}+g_{0}\right)^{3}}{\left(t / \epsilon_{r}+g_{0,(t=0)}\right)^{3}}},
\end{array}
$$


where $g_{0,(t=0)}$ is $g_{0}$ of the undeformed device. The change in $V_{\text {po }}$ can be found from the 1-D solution for pull-out (Eq. 1.10):

$$
\begin{aligned}
V_{\mathrm{po}}^{ \pm} \pm \sqrt{\frac{2 k g_{0}\left(t / \epsilon_{r}\right)^{2}}{\epsilon_{0} A}} \Longrightarrow & \\
& V_{\mathrm{po}}^{ \pm}=V_{\mathrm{po},(t=0)}^{ \pm} \sqrt{\frac{g_{0}}{g_{0,(t=0)}}} .
\end{aligned}
$$

From these two equations we can see that a change in $g_{0}$ will have a greater effect on $\left|V_{\mathrm{pi}}\right|$ than on $\left|V_{\mathrm{po}}\right|$. Also, when $g_{0}$ is equal to $\frac{t}{2 \epsilon_{r}},\left|V_{\mathrm{pi}}\right|$ becomes equal to $\left|V_{\mathrm{po}}\right|$ (the top plate touches the dielectric just as it is about to become unstable). For gaps smaller than this value, $\left|V_{\mathrm{pi}}\right|$ ceases to be a meaningful parameter, and the $C-V$ curve no longer exhibits hysteresis.

When $\left|V_{\mathrm{po}}\right|$ (normalized to $\left|V_{\mathrm{pi},(t=0)}\right|$ ) is plotted as function of the normalized $V_{\text {pi }}$ for increasing inhomogeneous charge $\sigma_{V_{\text {shift }}}$ and decreasing gap $g_{0}$, the lines as shown in Fig. 3.2 are obtained, which clearly indicate the different relation between $V_{\mathrm{pi}}$ and $V_{\text {po }}$ for the two degradation mechanisms. A pristine device will start somewhere along the line $\left|V_{\mathrm{pi}}\right| /\left|V_{\mathrm{pi},(t=0)}\right|=1$ and move along a solid line for pure inhomogeneous charging, or along a dotted line for pure mechanical degradation. Devices will not open anymore when (due to inhomogeneous charging) $\left|V_{\text {po }}\right|$ becomes zero, and ceases to exhibit hysteresis when (due to mechanical degradation) $\left|V_{\mathrm{pi}}\right|$ becomes equal to $\left|V_{\mathrm{po}}\right|$. From the graph we see that when we compare a device with a relatively low pull-out voltage (thin dielectric) to a device with a relatively high pull-out voltage, the device with low pull-out voltage can withstand more mechanical degradation. On the other hand, a low pull-out voltage makes a device more sensitive to inhomogeneous charging: a device with high pull-out voltage can withstand more inhomogeneous charging, but less mechanical degradation.

If $C-V$ curve narrowing is due to a combination of mechanical degradation and inhomogeneous charging, the terms $V_{\mathrm{pi},(t=0)}^{ \pm}$and $V_{\mathrm{po},(t=0)}^{ \pm}$of Eq. 3.11 and Eq. 3.12, can be substituted with $V_{\text {pi }}^{ \pm}$and $V_{\text {po }}^{ \pm}$of Eq. 3.14 and Eq. 3.15 to obtain the more general equations

$$
V_{\mathrm{pi}}^{ \pm}=\bar{V}_{\mathrm{shift}}+V_{\mathrm{pi},(t=0)}^{ \pm} \sqrt{\frac{\left(t / \epsilon_{r}+g_{0}\right)^{3}}{\left(t / \epsilon_{r}+g_{0,(t=0)}\right)^{3}}-\frac{\sigma_{V_{\text {shift }}}^{2}}{V_{\mathrm{pi},(t=0)}^{2}}},
$$

and

$$
V_{\mathrm{po}}^{ \pm}=\bar{V}_{\mathrm{shift}}+V_{\mathrm{po},(t=0)}^{ \pm} \sqrt{\frac{g_{0}}{g_{0,(t=0)}}-\frac{\sigma_{V_{\mathrm{shift}}}^{2}}{V_{\mathrm{po},(t=0)}^{2}}} .
$$




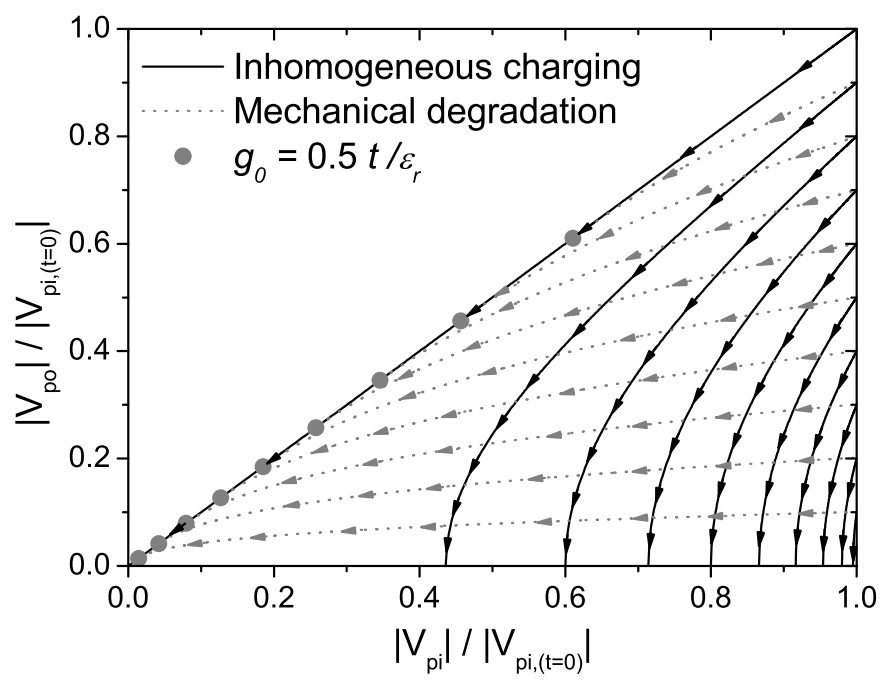

Figure 3.2: $\left|V_{\mathrm{po}}\right|$ (normalized to $\left|V_{\mathrm{pi}}\right|$ at $t=0$ ) as a function of normalized $\left|V_{\mathrm{pi}}\right|$ when a switch degrades. Arrows indicate the direction of increasing degradation. When $\left|V_{\mathrm{po}}\right|$ becomes zero, a device will not open anymore. The grey dots indicate when due to mechanical degradation $\left|V_{\mathrm{pi}}\right|$ is no longer a meaningful parameter.

$\bar{V}_{\text {shift }}$ can easily be established by measuring both the positive and negative pull-in voltage and then calculating $\left(V_{\mathrm{pi}}^{+}+V_{\mathrm{pi}}^{-}\right) / 2$. However, obtaining the two remaining degrading parameters, $\sigma_{V_{\text {shift }}}$ and $g_{0}$, from measured changes in pull-in voltage $\left(\left|V_{\mathrm{pi}}\right| / \mid V_{\mathrm{pi},(t=0)}\right)$ and pull-out voltage $\left(\left|V_{\mathrm{pi}}\right| / \mid V_{\mathrm{pi},(t=0)}\right)$ is a bit more complicated. It requires solving two coupled equations that are rather complicated, and although an analytical solution exists, finding this solution is best handled by symbolic mathematical computer programs such as Mathematica or Maple.

\subsubsection{Measurements}

To establish which mechanism is the most important for $C$ - $V$ curve narrowing of our switches, we measured the $C$ - $V$ curve as a function of the applied stress type and time. The $C-V$ curves were obtained using the system described in chapter 2.3 and ensures that $C-V$ curves are measured quickly enough (1 $C-V$ curve in $400 \mathrm{~ms}$ ) to avoid significant charging effects during the measurement of the $C-V$ curve. From these $C-V$ curves $V_{\text {pi }}^{ \pm}$and $V_{\text {po }}^{ \pm}$were extracted.

We used three different stress patterns: a DC bias voltage, a $1 \mathrm{~Hz}$ symmetric square wave (both actuated in a way that minimizes the closing velocity), and $200 \mathrm{~Hz}$ fast pulses (actuated with a high closing velocity). A difference in closing 

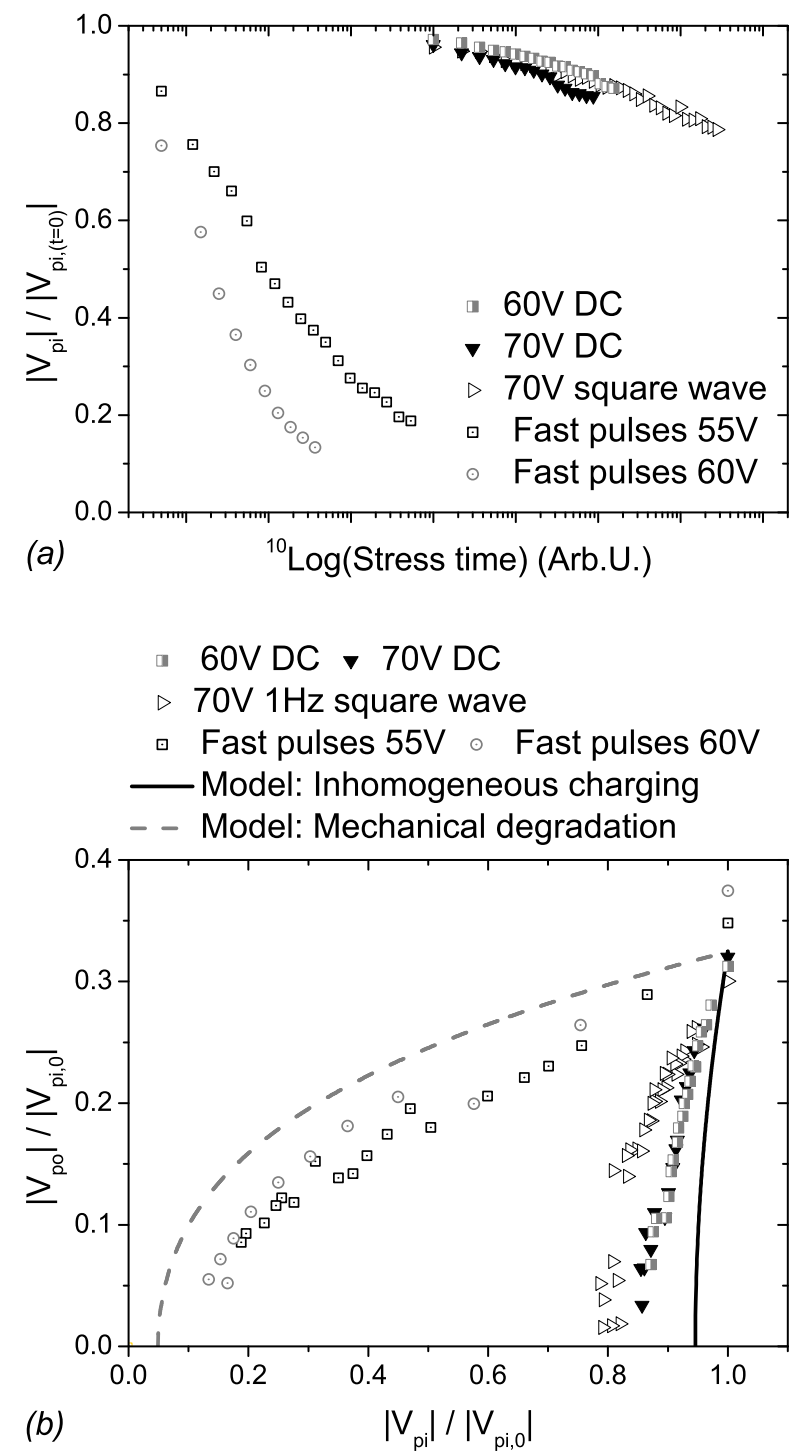

Figure 3.3: Measured changes in the width of the $C$ - $V$ curve. (a) $\left|V_{\mathrm{pi}}\right| /\left|V_{\mathrm{pi},(t=0)}\right|$ as a function of stress time. (b) $\left|V_{\mathrm{po}}\right| /\left|V_{\mathrm{pi},(t=0)}\right|$ as a function of $\left|V_{\mathrm{pi}}\right| /\left|V_{\mathrm{pi},(t=0)}\right|$. We define $\left|V_{\mathrm{pi}}\right|$ as $\left(V_{\mathrm{pi}}^{+}-V_{\mathrm{pi}}^{-}\right) / 2$. 
velocity was realized by changing the RC-time of the bias- $\mathrm{T}$ that was used to add the bias voltage to the RF signal used for measuring the capacitance. When the RC-time is much longer than the switching time, the switch closes while the voltage across the switch is equal to the pull-in voltage, whereas when the RC-time is much shorter than the switching time, the switch closes while the voltage across the switch is equal to the bias voltage. The latter can be significantly higher than the pull-in voltage, thus causing a higher closing velocity.

In Fig. 3.3a the normalized $V_{\mathrm{pi}}$ is plotted as function of stress time. We see that devices actuated with the $200 \mathrm{~Hz}$ fast pulses degrade much more rapidly than devices stressed with DC and square wave actuation. The reason for this large difference becomes clear in Fig. $3.3 \mathrm{~b}$, where $V_{\mathrm{po}}$ is plotted as a function of $V_{\mathrm{pi}}$, both normalized to $V_{\mathrm{pi},(t=0)}$. Here we see that in the DC bias voltage case the points lie close to the inhomogeneous charging line, indicating that the narrowing is primarily caused by inhomogeneous charging.

In the case of the fast pulses, results are much closer to the mechanical degradation line, indicating that narrowing is caused by mechanical degradation of the springs. The deviations from the theoretical lines could be caused by combined effects of inhomogeneous charging and mechanical degradation. It might also be caused by the way the switch is modeled: since we regard the top electrode as a rigid plate, higher order effects on $V_{\mathrm{pi}}$ and $V_{\mathrm{po}}$ such as roll-off are not taken into account.

The square wave actuation results are close to the inhomogeneous charging line, but not as close as the DC bias voltage results. This suggests that their $C-V$ curve narrowing is due to the combined effect of mechanical degradation and inhomogeneous charging.

The differences in degradation mechanisms can also be observed if stressed devices are allowed to relax (Fig 3.4): the narrowing due to the DC bias voltage is almost completely reversible, which indicates that the narrowing was not caused by plastic deformation of the springs, since that would be permanent. The damage due to the fast actuation pulses is permanent, strongly suggesting plastic deformation has occurred. Devices stressed with the square wave actuation are between the other two, and show only partial recovery.

From the measurements in Fig. 3.3 and 3.4 we can conclude that when the closing velocity increases and the switch is rapidly cycled, the main failure mode shifts from inhomogeneous charging to mechanical degradation. Lifetime is then no longer limited by the amount of time the switch is in the closed position, but by the number of stress cycles. 


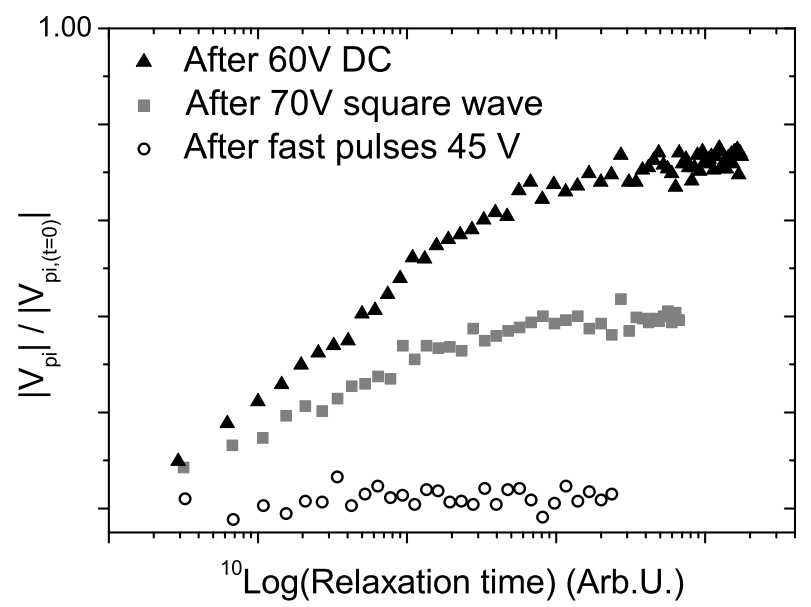

Figure 3.4: Relaxation of the width of the $C-V$ curve after narrowing.

\subsubsection{Conclusions about identification of degradation mech- anisms}

To conclude, we have introduced a new framework with which the relation between degradation mechanism, stress conditions, and actuation scheme can be identified. We used it to demonstrate for the first time that, depending on the stress conditions, degradation of RF MEMS capacitive switches does not only occur due to (inhomogeneous) dielectric charging, but can also originate in plastic deformations of the springs. Lifetime is then no longer limited by the time the switch is in the closed position, but by the number of stress cycles.

\subsection{Homogeneous dielectric charging}

In chapter 1.2 and 2.2 we saw that charge injection leads to a built-in voltage that shifts the $C-V$ curve. In section 3.2 we investigated the consequences of a builtin voltage in more detail, and saw that charge can be injected inhomogeneously, causing a narrowing effect. In this section we focus on the laterally averaged value of $V_{\text {shift }}(x, y)$ i.e. homogeneous dielectric charging.

Experiments have shown $[19,25,40]$ that $V_{\text {shift }}$ increases with time and stress voltage, but the exact mechanism and time behavior are not known. Although several models for the time and stress voltage dependence of the voltage shift have been proposed, the experimental verification of these models was usually done on a limited number of samples and/or over a small voltage and time range. Automation of the measurement system and an increase in the available material made 
it possible to study a large number of devices. Measurements on early development material made at NXP Semiconductors in Nijmegen showed (section 3.3.2) that the voltage shift of the $C$ - $V$-curve had a $\sqrt{t}$ dependence over a large time range. We further showed that the charging rate increases exponentially with the applied stress voltage, so that the time and voltage dependence of the shift in the $C$ - $V$-curve could be described by $V_{\text {shift }}=\alpha \sqrt{t} \exp \left(\beta V_{\text {stress }}\right)$.

More recently however, better devices and a more controlled actuation of the switches allow us to do experiments over a wider voltage and time range. Newer measurement show that the early $\sqrt{t}$ dependence is a special case of power law time dependence describing charging for relatively low voltages and/or short timescales. We also show that while originally assuming a stretched exponential was not warranted given the available measurement data, the newer measurements do conclusively show that at higher stress voltages and longer timescales, a stretched exponential time dependence gives a better description of the results than a pure power law time dependence.

\subsubsection{Measurement method and setup}

In section 2.2 we saw how the parabola method can be used to reliably determine $V_{\text {shift }}$. The experiments described in this chapter are measured using the RF setup of section 2.3 to do this more quickly. The experiments consist of a constant-voltage stress, interrupted at increasing time intervals for the low-voltage measurement of $V_{\text {shift }}$.

Because we defined $V_{\text {shift }}$ as the voltage needed for a minimal electrostatic force, it is not only determined by the amount of charge trapped during the stress test, but also by the work functions of the materials that are involved and the amount of charge trapped during plasma deposition of the dielectric. In the rest of this section we correct $V_{\text {shift }}$ for the value it has on $t=0$ :

$$
V_{\text {shift }} \rightarrow V_{\text {shift }}-V_{\text {shift },(t=0)} .
$$

This corrected $V_{\text {shift }}$ thus only accounts for charge trapped during the experiment. The measurements are conducted on unpackaged devices, with a dry $\mathrm{N}_{2}$ flow to prevent adverse effects due to moisture.

\subsubsection{Measurements and interpretation}

In Fig. 3.5a an example of the measured $V_{\text {shift }}$ as function of stress time is given. In this case the measured device was from early development material. On a doublelogarithmic scale (Fig. 3.5b), the points are approximately on a straight line with a slope around 0.56 .

It is therefore tempting to follow previous works in using a few-parameter model to describe the behavior of $V_{\text {shift }}(t)$ at a fixed stress voltage. Van Spengen et 


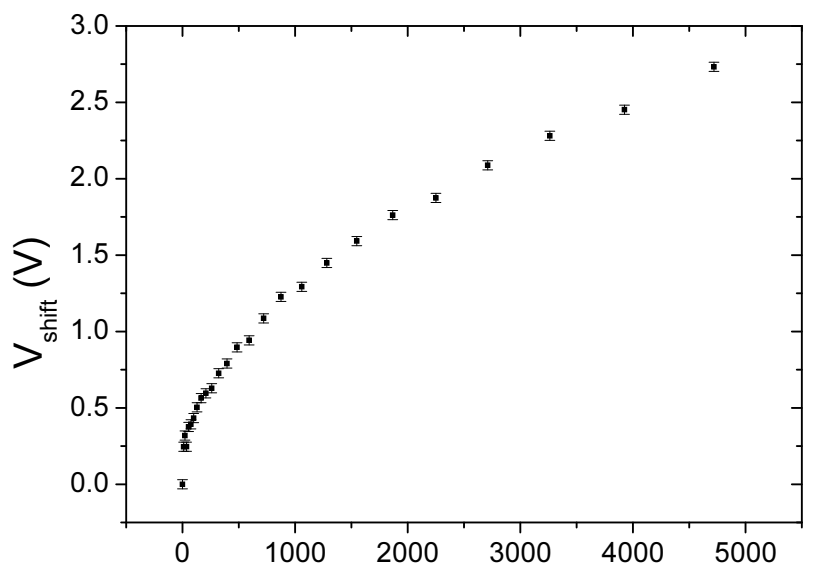

a)

Stress time (s)

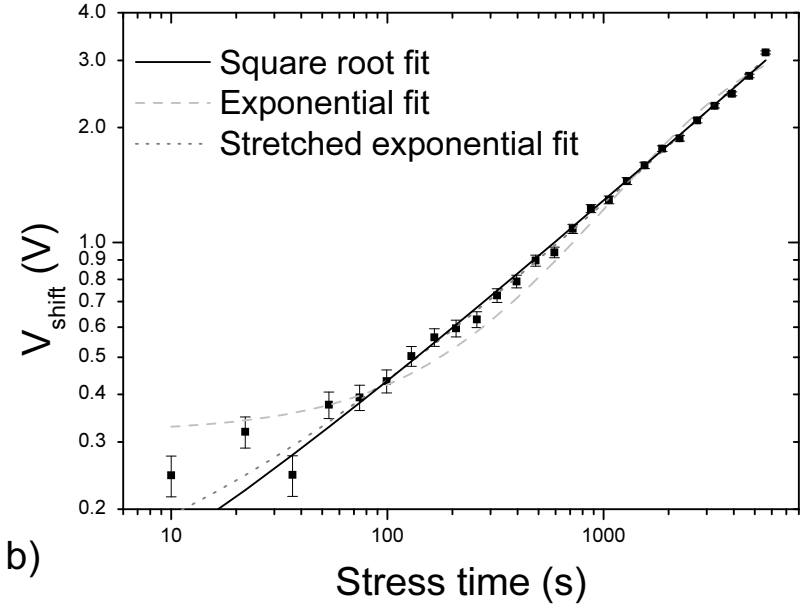

Figure 3.5: Measured $V_{\text {shift }}$ as a function of stress time. $V_{\text {stress }}: 40 \mathrm{~V}$. a) Linear axes. b) Double logarithmic axes with three different fit functions. 
al. [21], Yuan et al. [25], and Papaioannou et al. [26] describe charge build-up in the dielectric with an exponential time dependence indicating a fixed amount of traps which are slowly filled by the leakage current.

Since in the closed state the capacitive switch is similar to a MIM capacitor, reliability literature on these devices may also offer relevant models. Lau et al. [47], Shannon et al. [48], van Delden et al. [49], and Street [50] link degradation of amorphous silicon and silicon nitride to the generation of metastable defects by currents, with mechanisms related to light-induced changes in amorphous silicon $[51,52]$. They find that the defect density is proportional to the square root of time. Redfield et al. [53] and Zafar et al. [54] interpret generation of metastable defects in another way and propose a stretched-exponential time dependence.

Hence, we compare the three following time evolutions of $V_{\text {shift }}$ :

$$
\begin{aligned}
& V_{\text {shift }}(t)=V_{\max } \cdot(1-\exp (-k t))+V_{0} \quad(\text { Exponential }) \\
& V_{\text {shift }}(t)=a \sqrt{t}+V_{0} \quad \text { (Square root) } \\
& V_{\text {shift }}(t)=V_{\max } \cdot\left(1-\exp \left(-k t^{\delta}\right)\right)+V_{0} . \quad \text { (Stretched exponential) }
\end{aligned}
$$

In these equations $V_{\max }, k, a$ and $\delta$ may all depend on the stress voltage and temperature. The extra fit parameter $V_{0}$ was added to each model to account for small systematic errors in the determination of $V_{\text {shift }}$ - induced i.e. by the limited accuracy of the correction for $V_{\text {shift, }(t=0)}$ given by equation 3.18 .

The data in Fig. 3.5b lie approximately on a straight line with slope 0.56 , which hints in the direction of the square root behavior also found in the degradation of MIM capacitors. If we fit the function $V_{\text {shift }}(t)=a \sqrt{t}+V_{0}$ to the data we get a good agreement between the measurement data and the fitting result (see Table 3.1). The accuracy of each $V_{\text {shift }}$ data point from the parabolic fit procedure amounts to $30 \mathrm{mV}$.

Apart from the uncertainty of $V_{\text {shift }}$ due to the parabolic fit, there were also additional unquantified fluctuations, such as temperature variations: on very long time scales correlation between charging speed and temperature changes due to day/night cycles were observed. Also, there can be relaxation of the injected charge during the determination of $V_{\text {shift }}$ (on short time scales the time it takes to determine $V_{\text {shift }}$ becomes comparable to the stress time between two data points). The fit with the square root function suggests that the overall error per measurement is $50 \mathrm{mV}$. The fitted value of $V_{0}$ is $39 \pm 15 \mathrm{mV}$, which is small enough to be consistent with the notion that it is only added to account for the small systematic error made when $V_{\text {shift }}$ is corrected for its value on $t=0$.

In table 3.1, the fit results of the other two models are also presented. The $\chi^{2} /$ D.O.F. is a measure of the distance of the points to the curve (normalized to the measurement error) and a lower value objectively indicates a better fit, even if the measurement error is not known exactly. Clearly, the exponential fit gives a poor description of the data, while the other two models are satisfactory. However, 
Table 3.1: Fitting results of the measurement data displayed in Fig. 3.5b

\begin{tabular}{|c|c|c|}
\hline Model & $\frac{\chi^{2}}{\text { D.O.F. }}$ & $2^{*}$ Results \\
\hline$V_{\max } \cdot(1-\exp (-k t))+V_{0}$ & 13.6 & $\begin{array}{r}V_{\max }=3.01 \pm 0.05 \\
k=0.00036 \pm 0.00001 \\
V_{0}=0.32 \pm 0.01\end{array}$ \\
\hline$a \sqrt{t}+V_{0}$ & 2.6 & $\begin{array}{r}a=0.0395 \pm 0.0004 \\
V_{0}=0.039 \pm 0.015\end{array}$ \\
\hline$V_{\max } \cdot\left(1-\exp \left(-k t^{\delta}\right)\right)+V_{0}$ & 2.3 & $\begin{array}{r}V_{\text {max }}: \text { Does not converge } \\
k: \text { Does not converge } \\
\delta=0.53 \pm 0.03 \\
V_{0}=0.09 \pm 0.02\end{array}$ \\
\hline
\end{tabular}

the stretched-exponential equation fit does not converge to a final result: while $\delta$ converges to $0.53 \pm 0.03, V_{\max }$ and $k$ turn out to be highly correlated, with $k$ very small and $V_{\max }$ very large. Since $k$ is very small a Taylor expansion can be performed on (3.21):

$$
\begin{aligned}
V_{\text {shift }}(t)= & V_{\max } \cdot\left(1-\exp \left(-k t^{\delta}\right)\right)= \\
& V_{\max } \cdot\left(k t^{\delta}+\mathcal{O}\left(k^{2} t^{2 \delta}\right)\right) .
\end{aligned}
$$

If $\delta=0.5, V_{\max }=a / k$, and take the limit of $k \rightarrow 0$, the stretched exponential is equal to the square root (3.20). This indicates that the stretched exponential is in the limit where it converges to the square root function. We conclude that a stretched exponential is an unnecessary generalization of model 2 for these data.

The square-root model seems preferable, because of its good correspondence to the data and its simplicity. To verify if measurements at different stress voltages show the same $\sqrt{t}$ dependence, we plot $V_{\text {shift }}$ as a function of $\sqrt{t}$ for different voltages. This is shown in Fig. 3.6. For clarity, only 8 out of 62 measurements are shown here. The model holds well over the entire stress-bias regime, while it is again evident that the error-bars based on the parabolic fit uncertainty somewhat underestimate the real uncertainty.

From all 62 charging measurements the slope $a$ of the square root time dependence was obtained. It is plotted as a function of stress voltage in Fig. 3.7. Although there is quite some spread from device to device, from this graph it is clear that within the range of stress voltages the slope increases exponentially with $V$. This is in agreement with previous results which state that the lifetime decreases exponentially with $V$ [20]. We thus find that for this material the time and voltage behavior of dielectric charging in RF MEMS capacitive switches can 


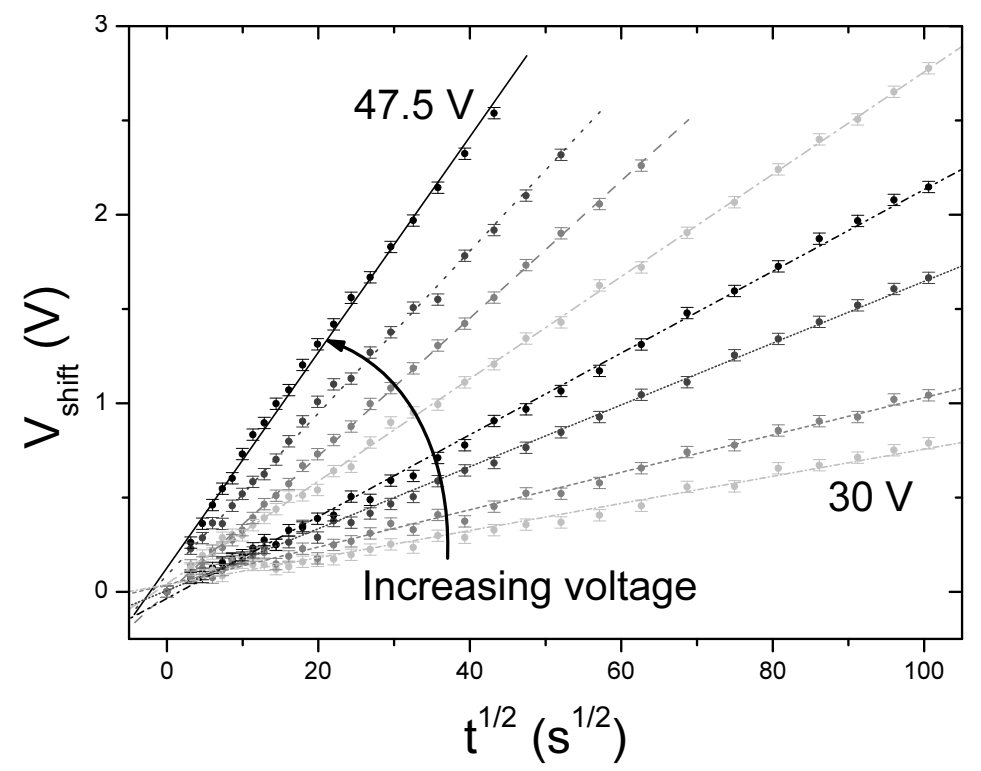

Figure 3.6: Shift of the $C$ - $V$-curve as a function of $\sqrt{t}$ for stress voltages ranging from $30 \mathrm{~V}$ to $47.5 \mathrm{~V}$, with $\Delta V=2.5 \mathrm{~V}$.

be described with the straightforward equation:

$$
V_{\text {shift }}(t)=\alpha \sqrt{t} \exp \left(\beta V_{\text {stress }}\right) \text {, }
$$

for which in our case $\alpha \simeq 2.4 \cdot 10^{-4} \mathrm{~V} / \mathrm{s}^{1 / 2}$ and $\beta \simeq 0.12 \mathrm{~V}^{-1}$. This equation makes it possible to make a fast reliability estimate based on data from an accelerated test.

As more material became available, additional measurements were done. Also, by testing with a slow bias-T, mechanical degradation, normally present when applying a high actuation voltage, could be mitigated, so that results over a wider applied voltage and $V_{\text {shift }}$ range were obtained. Fig. 3.8 shows the results of these newer stress tests. Although on a log-log scale the data points lie on a straight line in the lower left corner, a clear deviation from power-law behavior can be seen in the upper half of the graph. Also, the slope $\delta$ in the lower left corner is not 0.5 anymore, but lies between 0.37 and 0.41 . It does not exhibit an obvious correlation with the applied voltage. Due to these deviations, the $\sqrt{t}$ dependence, but also a more general power law dependence, do not yield good fits anymore. However, using a stretched exponential relation still does result in a good fit, and is fitted to the data in Fig. 3.8. Also, the stretched exponential relation is still compatible with the notion that charging is linked to generation of metastable defects. 


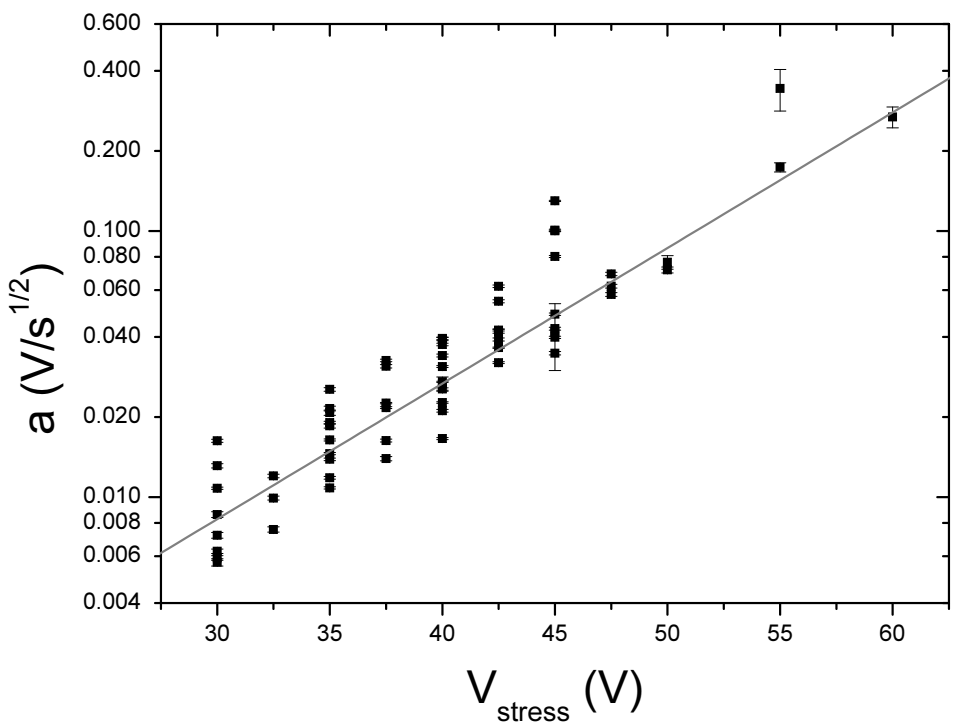

Figure 3.7: Fit parameter $a$ obtained from the data fitted with $V_{\text {shift }}(t)=a \sqrt{t}+V_{0}$ as function of the bias voltage with which the switches were stressed. The line is an exponential fit to the data of a cluster of closely spaced devices. 


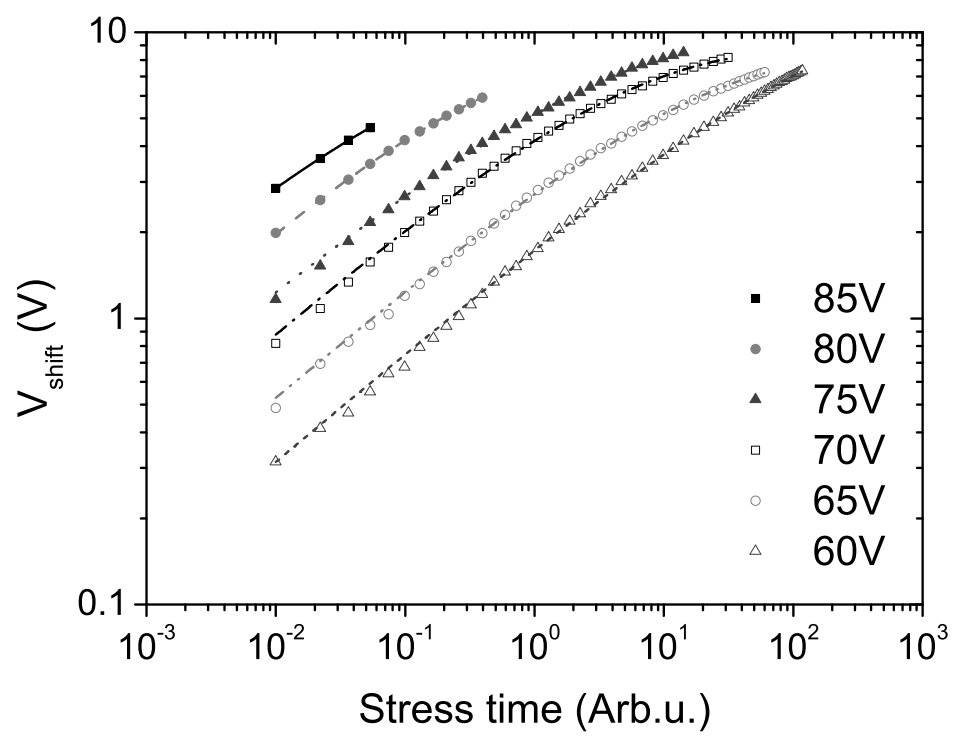

Figure 3.8: $V_{\text {shift }}$ as a function of stress time for stress voltages between $60 \mathrm{~V}$ and $85 \mathrm{~V}$. For high voltages and/or long stress times clear deviation from power law behavior is observed. Data is fitted with a stretched exponential, with the fit parameters plotted in Fig. 3.9 and 3.10 . 

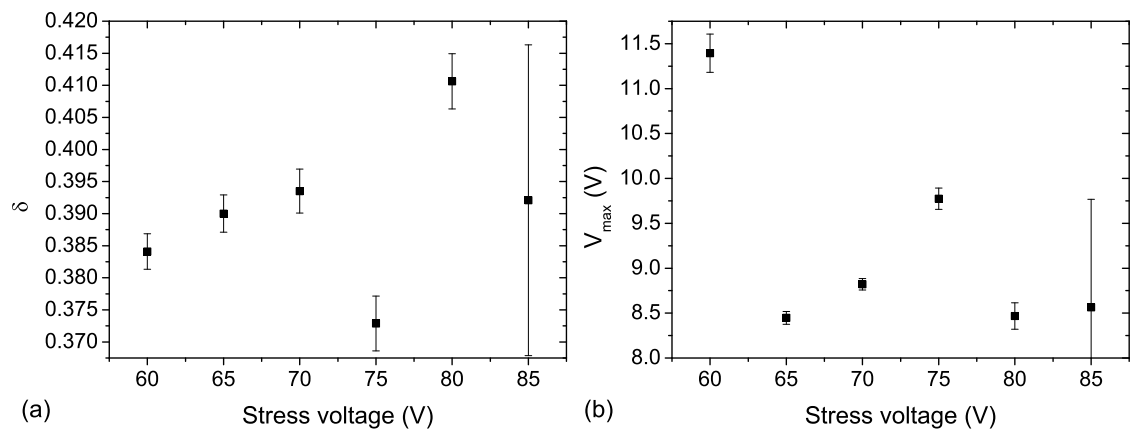

Figure 3.9: Fit parameters of the stretched exponential functions of Fig. 3.8 as a function of stress voltage. The error bars indicate no clear dependence on stress voltagee. (a) $\delta$. (b) $V_{\max }$.

As already mentioned, the fitted exponent does not exhibit an obvious correlation with the applied voltages, which is shown in Fig. 3.9. Here we also see that this is true for the parameter $V_{\max }$. This independence of $V_{\max }$ on the stress voltage makes it reasonable to assume that, for this nitride composition, the bend in $V_{\text {shift }}(t)$ on log-log scale is due to a saturation to some final value, and not due to a reversal caused by slow negative charging that compensates the positive charge. The latter situation is unlikely since it would require the negative and positive charging components to have the same voltage dependence if the maximum voltage is to be independent of voltage.

In Fig. 3.10 the fit parameter $k$ is plotted as a function of voltage. Just as we saw in Fig. 3.7 the relation between charging rate and voltage can be described by an exponential relation:

$$
k=\alpha \exp (\beta V),
$$

with $\alpha=(20 \pm 1) \cdot 10^{-5}$ and $\beta=0.106 \pm 0.007 \mathrm{~V}^{-1}$. Note that these figures cannot be directly compared with the earlier mentioned values because of the different value of $\delta(0.4$ versus 0.5$)$. Also, the choice for an exponential relation ( $V$ model in the figure) is only based on the notion that many failure mechanisms depend exponentially on stress intensity. However, literature about other electric field driven processes such as Time Dependent Dielectric Breakdown (TDDB) shows that the field dependence of degradation mechanisms in dielectrics is not a clear-cut case [55]. Following this, $k$ may be proportional to for instance $\exp \left(\beta^{\prime} V^{1 / 2}\right)\left(V^{1 / 2}\right.$ model $)$, or $\exp \left(-\beta^{\prime \prime} / V\right)(1 / V$ model $)$. Using these relations does not visually result in significantly better or worse fits. Doing a $\chi^{2}$ test to find out which model gives the best fits is problematic since the errors in $k$ are only fit parameter uncertainties: the 'real' error bars that would also take into account device variation would be much larger, but require a much greater amount of measurement data. Since an 


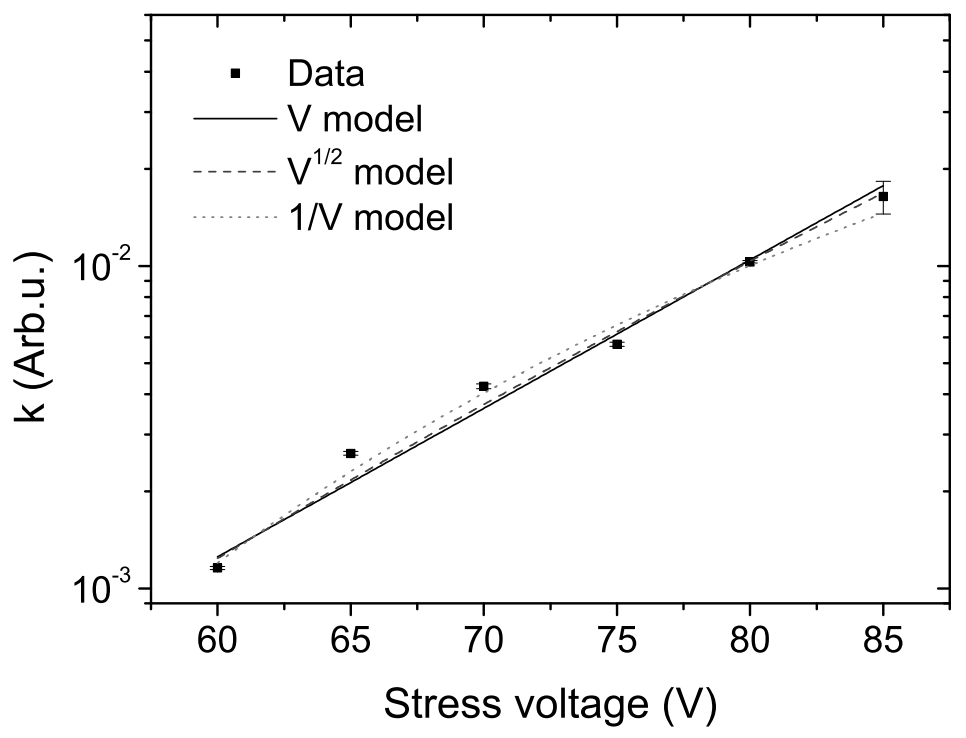

Figure 3.10: Fit parameter $k$ of the stretched exponential functions of Fig. 3.8 as a function of stress voltage, fitted with $V$ model, $V^{1 / 2}$ model and $1 / V$ model.

exponential relation is more conservative than the two other mentioned relations when extrapolating to lower voltage, it is apt to use this relation until new insights and measurements prove otherwise.

\subsubsection{Conclusions about homogeneous dielectric charging}

A thorough investigation of the charge injection in RF MEMS capacitive switches is presented. We collected a large amount of measurement data using a refined stress-measure sequence. The dielectric charging, expressed as the voltage shift of the minimum capacitance value, showed a dependence with the square-root of time over a wide range of stress conditions for early development material. More recent material shows that this behavior is a special case of the more general stretched exponential behavior. However, this can only be distinguished from power law behavior if a device is stressed long enough to induce a $V_{\text {shift }}$ of more than approximately $5 \mathrm{~V}$.

The voltage dependence of the charging rate can be described with a straightforward exponential relation for both the new (from the ICN6 fab Nijmegen) and the older (from MiPlaza fab Eindhoven)) material. However, current data is not conclusive if this is indeed the proper relation. Other relations, proposed for other field driven degradation mechanisms, cannot be ruled out on the basis of our data. 
Since an exponential relation is the most conservative, it is apt to use this until other relations really are proven to give a superior description of dielectric charging.

All in all, the fact that Eq. 3.21 gives a good description of the results, together with the results of reliability experiments on similar materials [49], is a strong indicator that dielectric charging is governed by charge trapping in current-induced metastable defects, which is a mechanism also held responsible for changes in the conductivity of silicon nitride MIM capacitors.

\subsection{Inhomogeneous dielectric charging}

Earlier in this chapter we saw that laterally inhomogeneous dielectric charging leads to a narrowing of the $C-V$ curve, and can lead to stiction of the membrane. In this section we show how Scanning Kelvin Probe Microscopy (SKPM) can be used to directly detect charge in the dielectric of RF MEMS capacitive switches, which enables us to study inhomogeneous charging in more detail. We observe a laterally inhomogeneous distribution. The measurements show that trapped charges slowly diffuse, which reduces the inhomogeneity and shows that charge is vertically confined. From these measurements we estimate the lateral diffusion coefficient of trapped charges.

\subsubsection{Scanning Kelvin Probe Microscopy}

Scanning Kelvin probe microscopy (SKPM) is a technique based on atomic force microscopy (AFM), first proposed by Nonnenmacher et al. [56]. With it, the contact potential difference $V_{\mathrm{CPD}}$ between a conducting tip and a surface can be measured. This difference is the sum of the difference in work function and the built-in voltage resulting from trapped charges. This makes it a powerful tool when studying properties of dielectrics for use in RF MEMS capacitive switches $[57,58]$. A schematical representation of an SKPM system is shown in Fig. 3.11.

A single SKPM line scan consists of two parts: measurement of the topology, and the actual measurement of the contact potential difference. The topology is measured using the AFM amplitude modulation technique. To measure the topology, the cantilever is excited piezoelectrically with an $\mathrm{AC}$ voltage at or near the resonance frequency of the cantilever. The amplitude and phase of the vibrating cantilever is typically detected with a four quadrant detector that registers laser light reflected from the tip. The tip is moved in the lateral direction, and simultaneously a feedback controller adjusts the height of the cantilever (with the same piezo crystal used to excite the cantilever) to keep the amplitude of the resonating cantilever constant. After this the tip is raised by about $100 \mathrm{~nm}$, after which the same line is scanned again, but this time the distance between tip and surface is adjusted with the obtained information during the first part of the line scan, and 


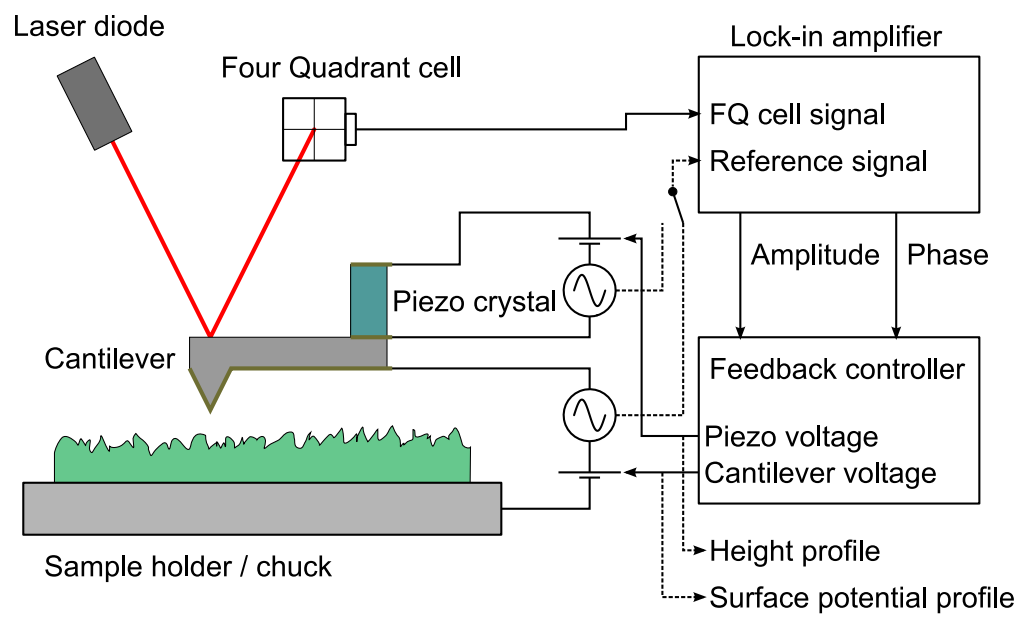

Figure 3.11: Schematic representation of a scanning kelvin probe microscope.

the cantilever is not excited by an AC piezo crystal voltage. Instead, the AC voltage (still at the mechanical resonance frequency) is applied to the conducting tip, which results in an electrostatic force very similar to Eq. 1.4, but with $V$ equal to $V_{\text {applied }}+V_{\mathrm{CPD}}$ and a different $u$-dependence of the capacitance:

$$
\begin{aligned}
F_{E}(u) & =\frac{1}{2}\left(V_{\text {applied }}+V_{\mathrm{CPD}}\right)^{2} \frac{\mathrm{d} C(u)}{\mathrm{d} u} \\
& =\frac{1}{2} \frac{\mathrm{d} C(u)}{\mathrm{d} u}\left(V_{\mathrm{DC}}+V_{\mathrm{AC}} \sin \left(\omega_{\mathrm{AC}} t\right)+V_{\mathrm{CPD}}\right)^{2} .
\end{aligned}
$$

From this equation we can see that the force will normally have a DC part, an AC part at the fundamental frequency $\omega$, and one at $2 \omega$ :

$$
\begin{aligned}
F_{E}(u)= & \frac{1}{2} \frac{\mathrm{d} C(u)}{\mathrm{d} u}\left[\left(V_{\mathrm{DC}}+V_{\mathrm{CPD}}\right)^{2}+\frac{V_{\mathrm{AC}}^{2}}{2}\right]+ \\
& \frac{\mathrm{d} C(u)}{\mathrm{d} u} V_{\mathrm{AC}}\left[V_{\mathrm{DC}}+V_{\mathrm{CPD}}\right] \sin (\omega t)+\frac{1}{4} \frac{\mathrm{d} C(u)}{\mathrm{d} u} V_{\mathrm{AC}}^{2} \cos (2 \omega t) .
\end{aligned}
$$

Since the AFM cantilever is a mechanical resonator with a high quality factor, it will for the most part only be excited by periodic forces at the fundamental frequency. Also, signals at different frequencies will be filtered out by the lock-in amplifier, so that $V_{\mathrm{CPD}}$ can now be determined by letting the feedback controller adjust $V_{\mathrm{DC}}$ to minimize the amplitude of the oscillating cantilever, since the minimum amplitude is reached if $V_{\mathrm{DC}}=-V_{\mathrm{CPD}}$. 


\subsubsection{Measurement results}

To investigate if laterally inhomogeneous charging indeed takes place, we applied a high stress voltage to a switch and waited until we had a significant amount of narrowing (between 5 and $10 \%$ change in $\left|V_{\mathrm{pi}}\right|$ ). After this we removed the top electrode, grounded the electrode beneath the dielectric and used SKPM to measure the surface potential, which was changed by the local built-in voltage shift, as a function of the position (Fig. 3.12). We clearly see where the holes in the top electrodes have been: since no charge was injected at that position, a lower surface potential is measured, although this is still higher than for an unstressed device, for which a surface potential of approximately zero was measured. It is also clear that the charge distribution is laterally inhomogeneous, with several 'hot-spots' with larger amounts of trapped charge.

To investigate how permanently charge is trapped in the dielectric (silicon nitride), we measured the surface potential of a device stressed at $70 \mathrm{~V}$ for 38 minutes along a single line as a function of time, resulting in Fig. 3.13. Here we not only see a gradual decay of the surface potential, but initially also an increase of the surface potential at the minima, indicating that charge flowed from the maxima to the minima. Later on, the surface potential also decreases at the minima. This tells us that initially changes in the surface potential are dominated by diffusion, and on a longer timescale by charge leaking back into the bottom electrode.

In Fig. 3.13 and 3.12 we also see that the characteristic length scale is $\sim 10 \mu \mathrm{m}$. Since the thickness of the dielectric is much smaller than this, charge must be vertically confined or the change in the surface potential would be dominated by vertical diffusion and leakage into the bottom electrode, which would only lead to an overall decay of the trapped charge.

\subsubsection{Estimation of the diffusion coefficient}

With the measurements shown in Fig. 3.13 we can also try to estimate the diffusion coefficient of the diffusing charge. The $2 \mathrm{D}$ diffusion equation is given by

$$
\frac{\partial V(x, y, t)}{\partial t}=D\left(\frac{\partial^{2}}{\partial x^{2}}+\frac{\partial^{2}}{\partial y^{2}}\right) V(x, y, t),
$$

where $D$ is the diffusion coefficient. Since we only have data in the $x$-direction (measuring in $2 \mathrm{D}$ is not an option, it is too slow with respect to the diffusion time-scale), we have to make an assumption about the second derivative to $y$. By using a portion of the data where the second derivative to $x$ is maximal, the second derivative to $y$ will most likely be equal or smaller in comparison. This region is indicated by the vertical lines in Fig. 3.13. D is then equal to 0.5 to 1 times the ratio of $\partial V(x, t) / \partial t$ and $\partial^{2} V(x, t) / \partial x^{2}$.

However, there is simultaneous diffusion and charge leakage back into the bottom electrode. To correct for this, we assume that if the surface potential is 

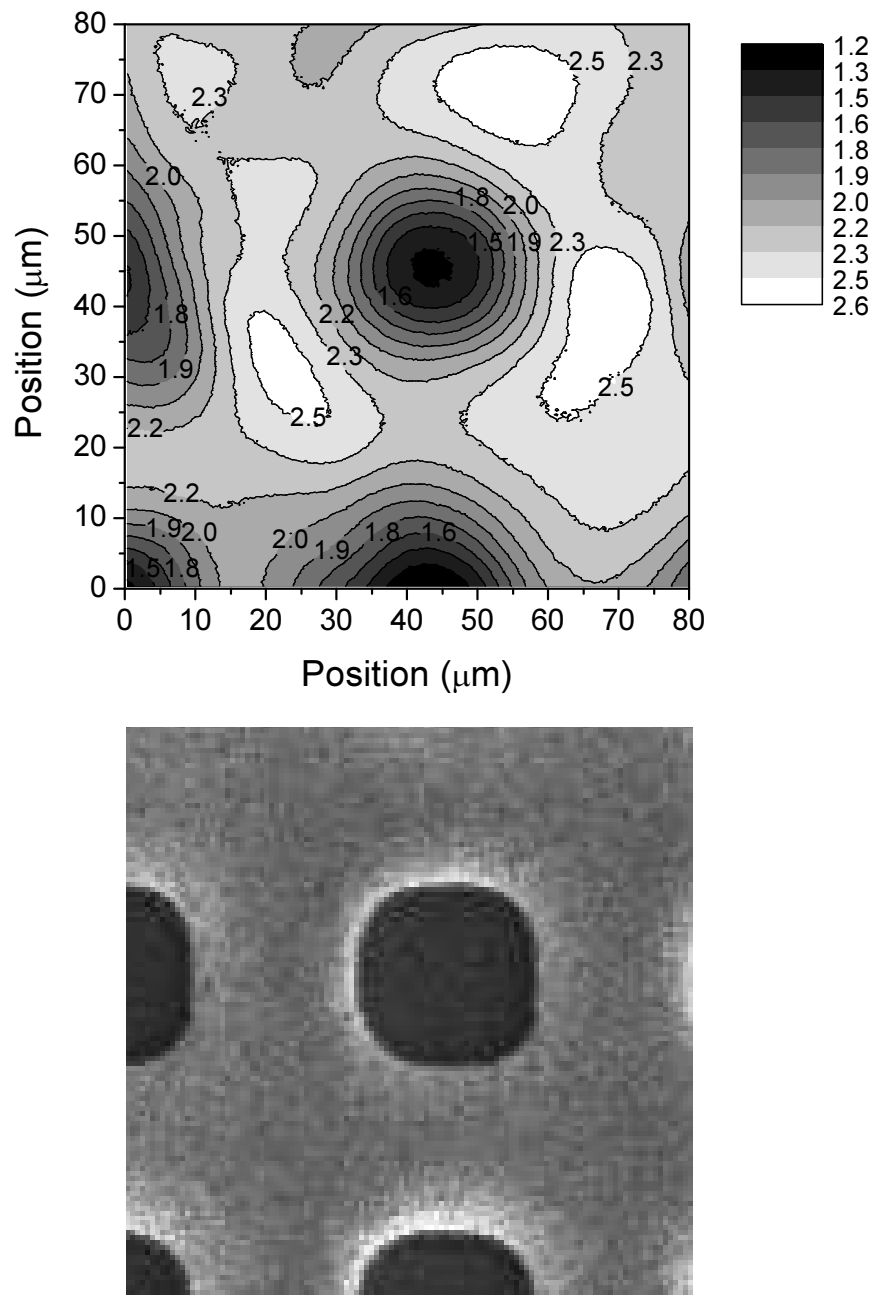

Figure 3.12: Contour plot of the measured surface potential of a stressed device and the corresponding section of a SEM picture of the top electrode. 


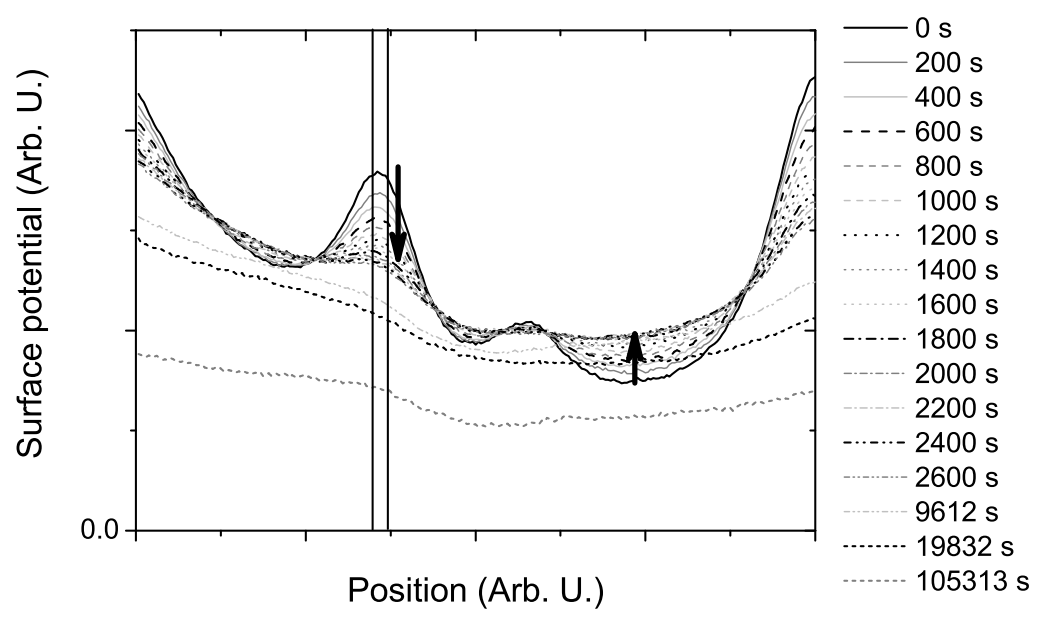

Figure 3.13: Diffusing and decaying surface potential measured across a line. The vertical lines indicate the region used for determination of the diffusion coefficient.

measured over a long line, that the diffusion in the $y$-direction in and out of this line averaged over the whole line is approximately zero, so that the change in the average surface potential $\overline{V(x, t)}$ is only due to leaking, and not due to diffusion in the $y$-direction. We also assume that the leak rate is proportional to the surface potential $V(x, t)$ i.e. ohmic conduction to the bottom electrode. The corrected expression for $\partial V(x, t) / \partial t$ then becomes

$$
\frac{\partial V(x, t)}{\partial t} \rightarrow \frac{\partial V(x, t)}{\partial t}-\frac{\partial \overline{V(x, t)}}{\partial t} \cdot \frac{V(x, t)}{\overline{V(x, t)}}
$$

To reduce noise, we convolute the second derivative to $x$ with a smoothing function. After averaging the result over the region indicated in Fig. 3.13 and over the time of the experiment, a diffusion coefficient between $0.0030 \pm 0.0005$ and $0.006 \pm$ $0.001 \mu \mathrm{m}^{2} / \mathrm{s}$ is found.

Since the second order derivative introduces a lot of noise, a second approach was used for another data set. This set was obtained in the same way as the data in Fig. 3.13 (this time 9 minutes of $80 \mathrm{~V}$ stress), but had less sharp features, because the shape of the surface potential in Fig. 3.13 was mostly determined by the fact that a hole was situated above the measured line of the dielectric. In order to determine $D$, we look at the largest Fourier component of the surface potential, which, when combined with an exponential time dependence, is a solution to the 


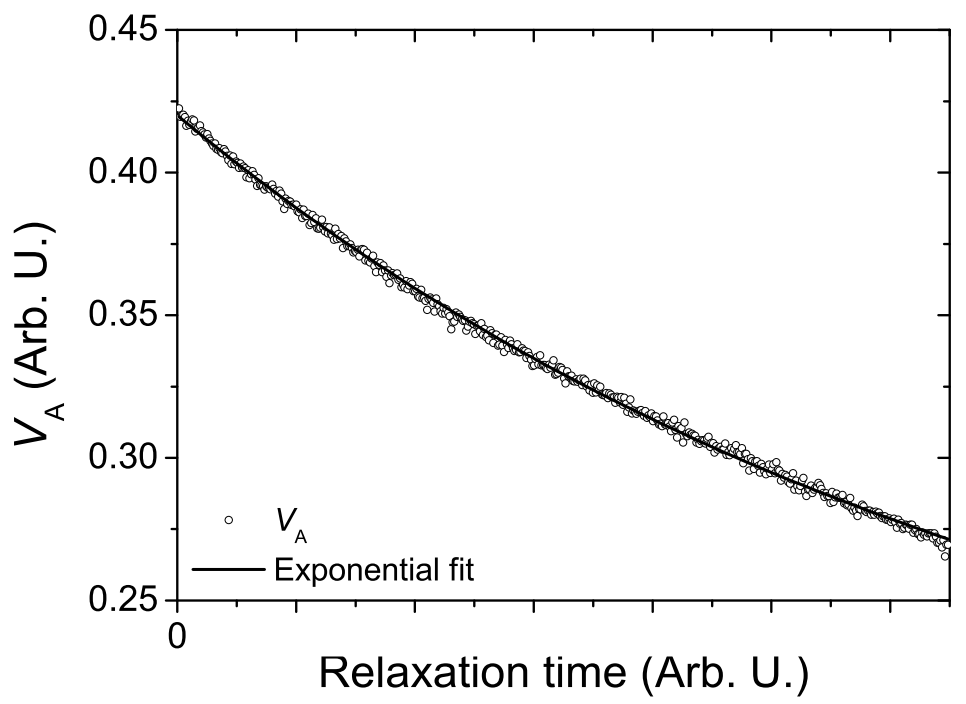

Figure 3.14: Exponential decay of the amplitude of a sine function fitted to a diffusing surface potential.

diffusion equation:

$$
\begin{aligned}
& V(x, y, t)=V_{\text {offset }}+ \\
& \quad V_{0} \sin \left(k_{x} x+\phi_{x}\right) \sin \left(k_{y} y+\phi_{y}\right) \exp \left(-D\left(k_{x}^{2}+k_{y}^{2}\right) t\right) .
\end{aligned}
$$

The $x$-dependent part can be determined by fitting the sine function $V_{A} \sin \left(k_{x} x+\right.$ $\phi)+V_{\text {offset }}+g_{\mathrm{L}} x$ to the data $\left(g_{\mathrm{L}}\right.$ is added to eliminate long distance gradients). We then plot $V_{A}$ as a function of time (Fig. 3.14). The extracted $k_{x}$ 's are not a function of time and are in good agreement with the hole distance of the top electrode. Again we have to make an assumption about what happens in the $y$-direction. Since the hole distance in the $y$-direction is equal to the hole distance in the $x$-direction, we expect similar behavior of the surface potential in that direction. We therefore presume $k_{y}=k_{x}$ and fit $V_{A}(t)=V_{0} \exp \left(-2 D k_{x}^{2} t\right)$ to the graph, which results in a diffusion coefficient $D$ of $0.010 \mu \mathrm{m}^{2} / \mathrm{s}$. This is higher than the other result because this method does not correct for the simultaneous leakage of charge into the bottom electrode, and also does not correct for higher order Fourier components of the diffusion in the $y$-direction. Since both methods use 1D data to calculate the diffusion coefficient for a $2 \mathrm{D}$ diffusion process, both are approximate values.

Since diffusion of trapped charge reduces the inhomogeneity of the charge distribution, another way to measure this is measuring the relaxation of the narrowing of the $C$ - $V$ curve. Fig. 3.15 shows the decay of $\sigma_{V_{\text {shift }}}$ as calculated from the change 


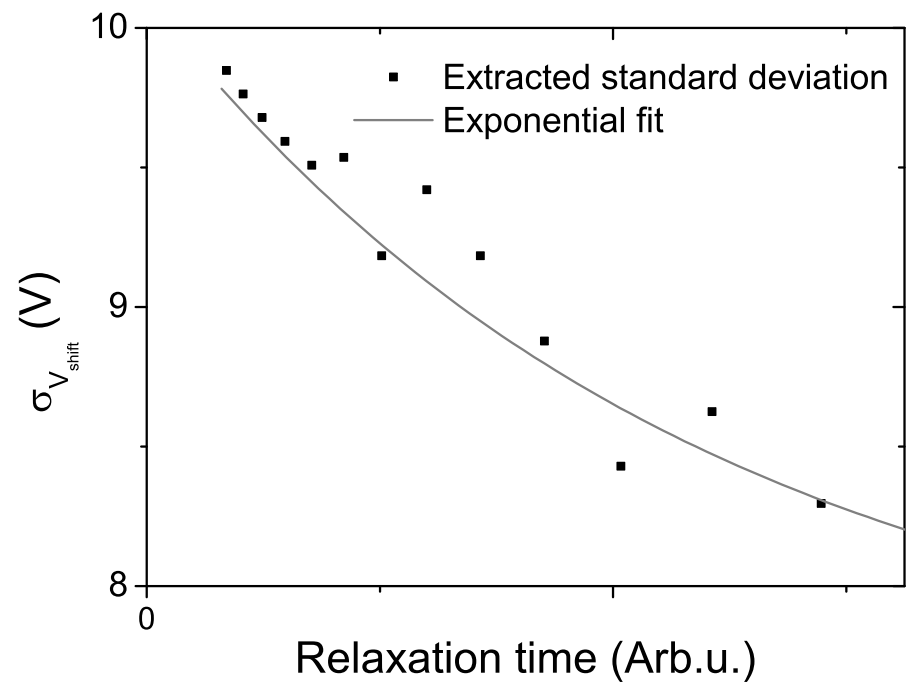

Figure 3.15: Decay of $\sigma_{V_{\text {shift }}}$. Time-scale is the same as in Fig. 3.14.

in $V_{\mathrm{pi}}$ after it has been stressed for approximately 10 hours at $45 \mathrm{~V}$. When we fit an exponential curve through the data (over a similar amount of relaxation time), we find a characteristic time scale that is 1.5 times shorter as the one found from the decaying amplitude of Fig. 3.14. This again shows that although results are not the same, they are in the same order of magnitude. It confirms that on shorter time scales relaxation of a narrowed $C-V$ curve is dominated by diffusion of trapped charge.

Of course the question remains whether the found diffusion coefficient is comparable to what could be expected from literature, and is the use of the diffusion equation warranted. The transport of mobile charge over the surface of a dielectric is not well charted. Theoretical studies are limited; two interesting papers in this respect are by Wintle et al. [59,60]. They discuss which forms of time dependence are possible for a surface charge that flows to an edge. They derive these equations from the continuity equation for moving charges, and on constitutive equations for the surface current that depend on whether a surface has a natural surface conductivity. It can be shown that if we apply their reasoning to our case, we can find the diffusion equation if the surface has a natural surface conductivity, and a nonlinear partial differential equation that resembles the diffusion equation if currents are charge driven. 
The continuity equation for moving charges in a plane is given by

$$
\vec{\nabla} \cdot \vec{J}(x, y, t)+\frac{\partial \sigma_{d}(x, y, t)}{\partial t}=0
$$

where $\nabla$ is the $2 \mathrm{D}$ vector differential operator. If there is a natural (ohmic) surface conductivity, $\vec{J}$ is linearly proportional to $\vec{E}$ :

$$
\vec{J}(x, y, t)=c \vec{E}(x, y, t) .
$$

However, if there is no built-in mechanism for surface conduction, only the excess charge can move under the influence of the $E$-field [59], with the velocity controlled by the mobility $\mu$ :

$$
\vec{J}(x, y, t)=\mu \sigma_{d}(x, y, t) \vec{E}(x, y, t)
$$

Finally, we need the relation between $\sigma_{d}$ and $\vec{E}$. This is specific to the geometry of the problem, and therefore different from the case Wintle describes: in our case an electrode at $0 \mathrm{~V}$ is located beneath the dielectric, whereas the Wintle case discusses a Faraday cage around the sample, connected at one end to the charged surface. Since by definition the electric field in the lateral direction is $\vec{E}(x, y, t)=-\vec{\nabla} V(x, y, t)$, together with Eq. 3.9 and the assumption that there is no bulk charge, we get for the electric field at the surface:

$$
\vec{E}(x, y, t)=-\vec{\nabla} V_{\text {shift }}(x, y, t)=-\vec{\nabla} \frac{t \sigma_{d}(x, y, t)}{\epsilon_{0} \epsilon_{r}}
$$

When we combine Equations 3.30, 3.31, and 3.33 we get:

$$
\frac{\partial V(x, y, t)}{\partial t}=\frac{t c}{\epsilon_{0} \epsilon_{r}} \nabla^{2} V(x, y, t)
$$

which is the diffusion equation we used. However, if instead Eq. 3.32 governs the surface conduction, we find:

$$
\frac{\partial V(x, y, t)}{\partial t}=\mu \vec{\nabla} \cdot(V(x, y, t) \vec{\nabla} V(x, y, t)) .
$$

As can be seen, this is a non-linear equation, and more difficult to tackle than the diffusion equation. If one were to find a self-similar spatial solution (like the harmonic spatial profiles are for the diffusion equation), the time dependence no longer follows an exponential decay. This will also be the case if there is a natural surface conduction mechanism which does not follow an ohmic relation. However, all these cases qualitatively look like diffusion: charge flows from regions with high concentration to regions with low concentration with a rate proportional to the 
gradient. Given the limitations of the measurement system, using the diffusion equation is a good first order approximation.

Experimentally the topic has proven difficult to access. In general, literature reports treating conduction on dielectric surfaces exhibit uncertainty over the type of charge carrier (ionic, electronic), its density, and (hence) its mobility. Recent literature on this subject mainly focuses on the conductivity of a dielectric surface under photon irradiation in vacuum. The photons stem from a UV light source or a synchrotron. The surface will exhibit the photo-electric effect, leading to (positive) surface charge-up. The electron yield from photon irradiation is typically not quantified in these experiments. Dielectric surfaces such as $\mathrm{KH}_{2} \mathrm{PO}_{4}$ [61], $\mathrm{KH}_{2} \mathrm{AsO}_{4}$ [61], $\mathrm{RbH}_{2} \mathrm{PO}_{4}$ [62], $\mathrm{SiO}_{2}$ [63], $\mathrm{MgO}$ [64], and mica [65] were studied in this manner. The measurements focus on surface conductivity as a function of photon flux and temperature.

As already mentioned, SKPM measurements on the same type of material were done at LAAS CNRS $[57,58]$. They did not observe diffusion, but this can be explained by the fact that they used a thinner, more leaky silicon nitride. This resulted in a typical discharge time of just a few hundred seconds, significantly shorter than the timescales at which charge leaks away in our material. Charge can thus leak away before it has a chance to diffuse.

From what we have been able to find, quantified measurements of surface charge transport on a material most similar to our dielectric were done by Buh et al. [66]. They do not use the same materials, but it does concern a silicon-based dielectric: their article reports a diffusion coefficient of holes on a $\mathrm{SiO}_{2}$ surface of $0.00035 \mu \mathrm{m}^{2} / \mathrm{s}$. Their figure for the diffusion coefficient is a factor 10-30 lower than our case, but this seems quite reasonable if one keeps in mind that the two materials have not been produced in the same way, and are chemically different (although a thin $\mathrm{SiO}_{2}$ layer is to be expected on top of the $\mathrm{Si}_{\mathrm{x}} \mathrm{N}_{\mathrm{y}}: \mathrm{H}$ dielectric [67]).

\subsubsection{Conclusions about inhomogeneous charging}

We can conclude that Scanning Kelvin Probe Microscopy is a useful tool to study charging in RF MEMS capacitive switches. It was used to show that charges are trapped in a laterally inhomogeneous way. This inhomogeneity is partly due to the topology of the top electrode (at the positions of the holes, less charge is transfered from the top electrode to the dielectric), but also has a random contribution, possibly due to variations in material composition.

Furthermore, time dependent measurements showed that the trapped charges are confined in the vertical direction and exhibit lateral diffusion. These measurements were used to get an estimated diffusion coefficient between 0.003 and $0.010 \mu \mathrm{m}^{2} / \mathrm{s}$. Although not much data is available on charge diffusing on a silicon nitride dielectric, it seems a reasonable figure when comparing it to the results of [66]. Following the modeling methodology used in [59], we showed how ohmic 
self-drift results in the used diffusion equation. Given the limitations of the measurement system and the current lack of more detailed knowledge about silicon nitride surface conduction, using the diffusion equation is a good first order approximation.

\subsection{Mechanical degradation}

In paragraph 3.2 we saw that rapid cycling of a switch with high closing velocity can lead to mechanical degradation. In order to produce a capacitive switch that both performs well and is reliable, it is important to know how reliability depends on the design and the circumstances under which it is used. In this section we will investigate how design, pressure, and applied voltage influence the degradation of the switches.

\subsubsection{Characterizing mechanical degradation}

To characterize mechanical degradation we use the system of section 2.3. Since we are interested in cycling effects a waveform is chosen that closes the switch as often as possible, but still allows the switch to completely open during the times at which the voltage is zero. In practice, this allows for 400 to 4000 closing and opening cycles per second, depending on the design of the switch and the pressure at which the switch is used. To minimize charging effects, bipolar actuation is used [18].

At the beginning of each test, the closing and opening transients are measured. From this we can calculate the (area averaged) position of the top electrode as a function of time. For this we assume that the position can be approximately calculated from the capacitance using the parallel plate capacitor formula, with a small correction for fringing of the electric field at the sides and the holes of the plate. This correction is done using the fringing model proposed by Suy et al. [5].

Alternatively, an optical profiler could be used to measure changes in the gap. However, this is not very suitable for measuring transient behavior, as this technique is much slower. We did on another occasion use such a profiler to measure static changes in the gap due to mechanical degradation, and found that the results were in agreement with the electrical measurements of the open state of the capacitive switch.

\subsubsection{Measurement results}

Fig. 3.16 shows the results of mechanical degradation experiments and the first measured closing transients for a number of closing voltages. In Fig. 3.17 the amount of holes in the top electrode has been varied. For the transients in Fig. 3.16 the maximum closing velocity has also been extracted, displayed in the degradation 

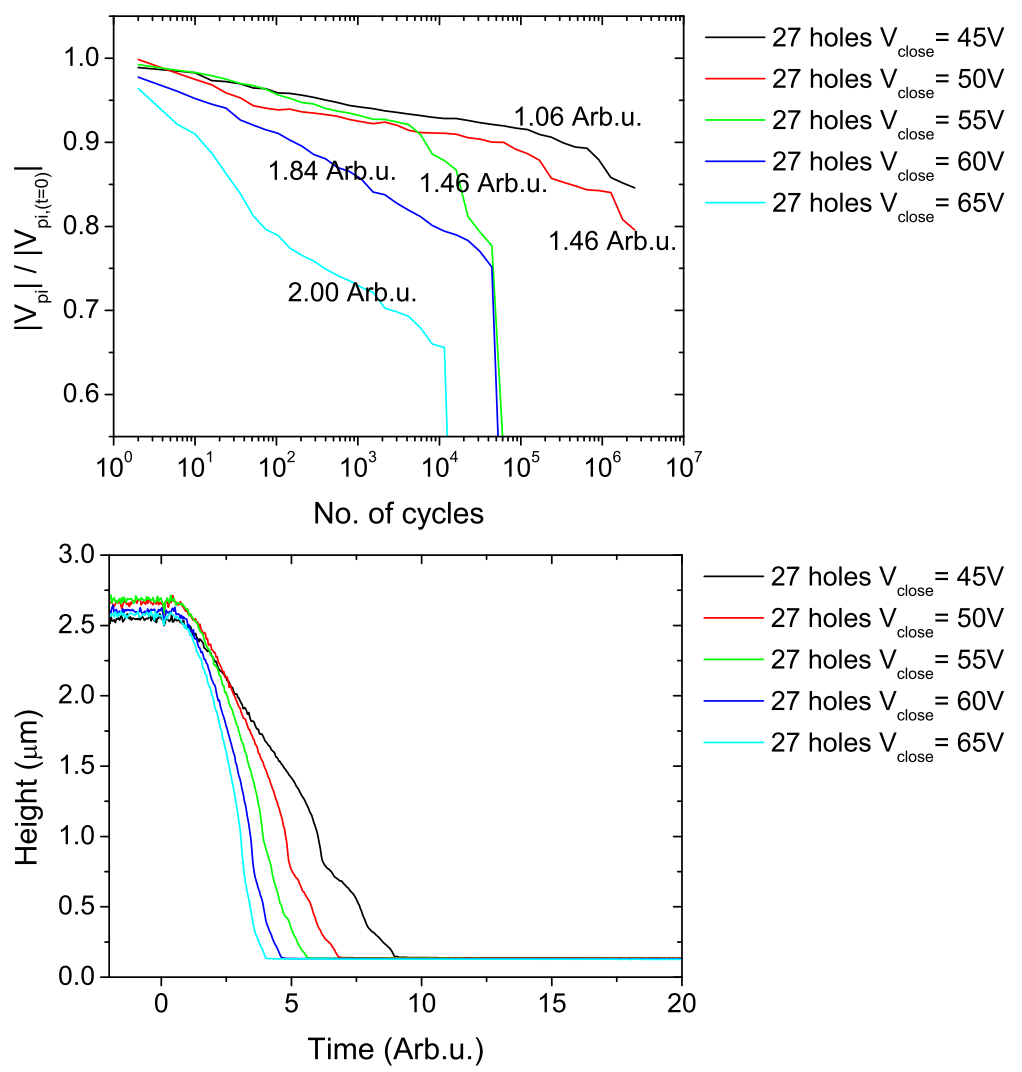

Figure 3.16: Degradation of $V_{\mathrm{pi}}$ as a function of time and closing transients for several actuation voltages. Used device: $480 \times 180 \mu m$ with $3 \times 9$ holes.

graph above it in arbitrary units. For Fig. 3.17 this is problematic because it is measured with a lower sampling rate, so that the fastest changes in capacitance are recorded less accurate. Because of this the maximum velocity is underestimated.

As expected, a higher actuation voltage greatly reduces the closing time, but simultaneously also increases the observed degradation. The same is true when the amount of holes is increased: the amount of squeeze-film damping is reduced, thereby decreasing the closing time but also increasing the degradation. Furthermore, the degradation seems to begin with an initial gradual degradation phase, that suddenly ends in a sharp and rapid reduction of $V_{\mathrm{pi}}$. The initial gradual degradation is fairly reproducible, but the moment at which the sudden jump in $V_{\text {pi }}$ happens has a lot more spread. This behavior will be discussed in more detail in chapter 4 . 


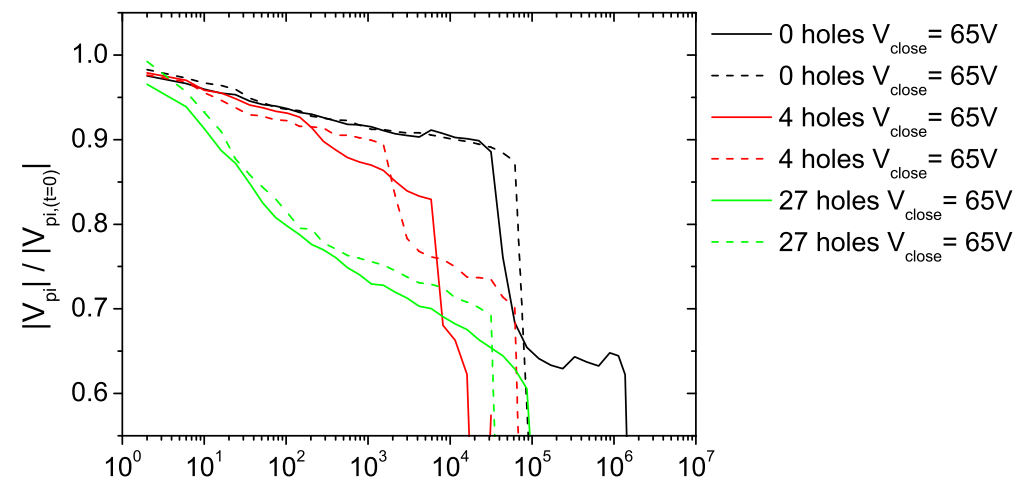

No. of cycles

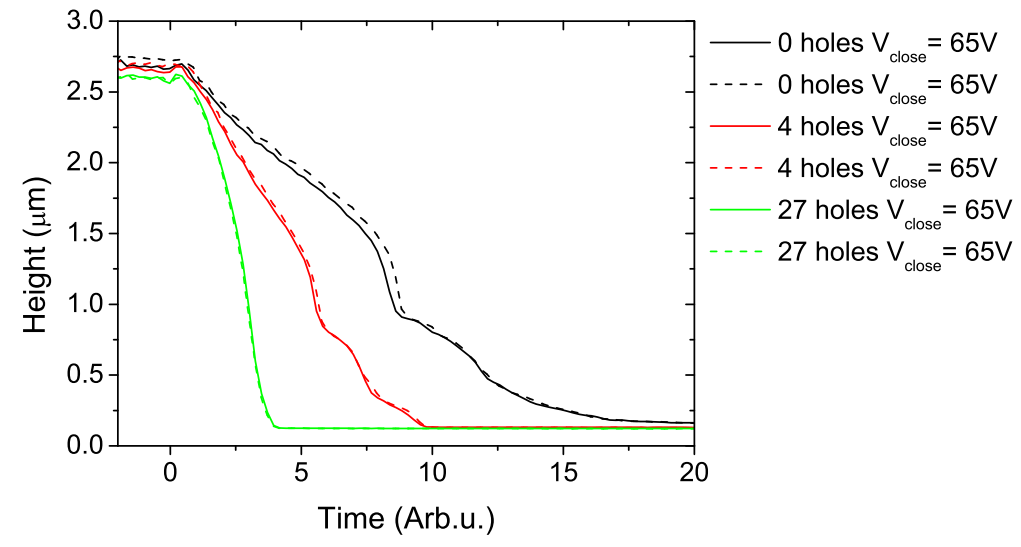

Figure 3.17: Degradation of $V_{\mathrm{pi}}$ as a function of time and closing transients for several devices: $480 \times 180 \mu \mathrm{m}$ with 0 holes, $1 \times 4$ holes, and $3 \times 9$ holes. 
Since both decreasing the actuation voltage and reducing the amount of holes in the top electrode reduces the degradation, the question rises which method has a better trade-off with respect to the closing time. For this the results of the no and 4 holes devices actuated at $65 \mathrm{~V}$ is plotted together with the results of the 27 holes devices actuated at 45, 50, and $55 \mathrm{~V}$. This is shown in Fig. 3.18. Here we see that the device with 4 holes actuated at $65 \mathrm{~V}$ degrades the most, but that the closing time is approximately equal to a 27 holes device actuated at $45 \mathrm{~V}$, which exhibits the least amount of degradation of the measured devices. The devices with no holes that were actuated at $65 \mathrm{~V}$ degrade a bit less, but are only as good as a 27 holes device actuated at $50 \mathrm{~V}$, while the closing time is much longer.

A possible explanation for these differences in degradation rates can be found upon closer examination of the closing transients: the transients of the 27 holes devices are smoother than the ones of the no holes and 4 holes devices. The latter have distinct bends in the transients. A clear example of this is visible in the closing transient of the no holes devices around $t=18 \mu \mathrm{s}$. At the distinct bends the second derivative to time is higher, which of course means that the top plate of the device undergoes a high acceleration. Another advantage of the 27 holes switch with respect to the 4 and no holes switches is that it opens a lot faster.

Besides actuation voltage and design layout, another factor in mechanical degradation is the pressure at which a switch is used. This can be seen in Fig. 3.19, which shows the amount of $V_{\mathrm{pi}}$ degradation as a function of the number of switching cycles for pressures between 0 and $1000 \mathrm{mBar}$ for an actuation voltage of $35 \mathrm{~V}$. The device under test is an older design from an older wafer than the ones used in other mechanical degradation tests, which makes it a bit more prone to mechanical degradation than the other devices. Again, the differences are striking: reducing the pressure decreases the closing as well as the opening times, but also increases the degradation rate. Note that although no open transients are shown here, other measurements we conducted clearly show a reduction of the opening time with decreasing pressure, which is a logical result of the reduced squeeze film damping due to rarefaction effects [15].

\subsubsection{Conclusions about mechanical degradation}

In the previous subsection we saw that variations in design can lead to differences in closing transients. This influences the degradation behavior of the switch: increasing squeeze-film damping by decreasing the amount of holes in the top electrode decreases the degradation rate. Alternatively, mechanical degradation can be reduced by lowering the actuation voltage. Measured closing transients together with mechanical degradation results show that even though the closing time is similar, the transient itself can be dissimilar enough to cause substantial differences in degradation rate. Experiments on the pressure dependence of mechanical degradation show that this too can have a large influence. 


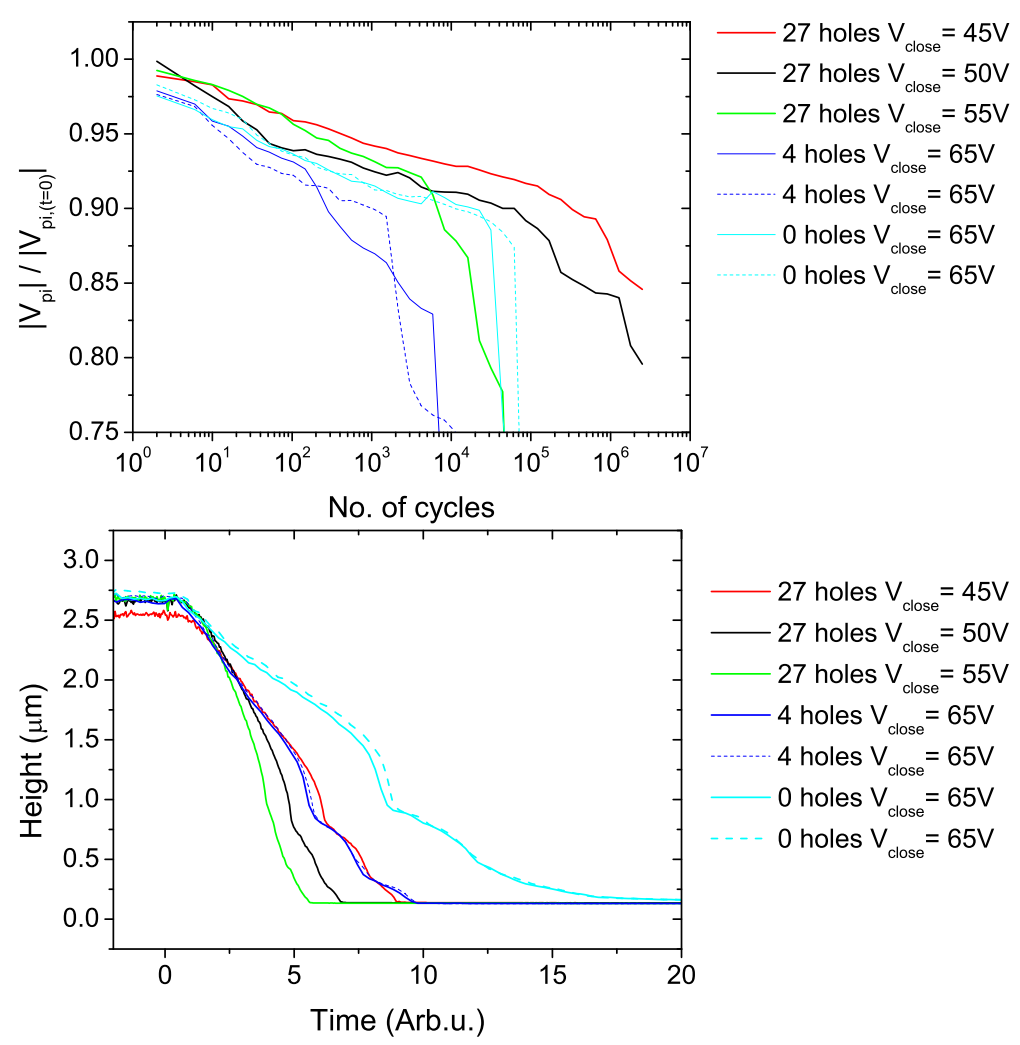

Figure 3.18: Degradation of $V_{\mathrm{pi}}$ as a function of time and closing transients for several devices: $480 \times 180 \mu \mathrm{m}$ with 0 and $1 \times 4$ holes at $65 \mathrm{~V}$, and $3 \times 9$ holes at 45,50 , and $55 \mathrm{~V}$. The data are selected from Figs. 3.16 and 3.17. 


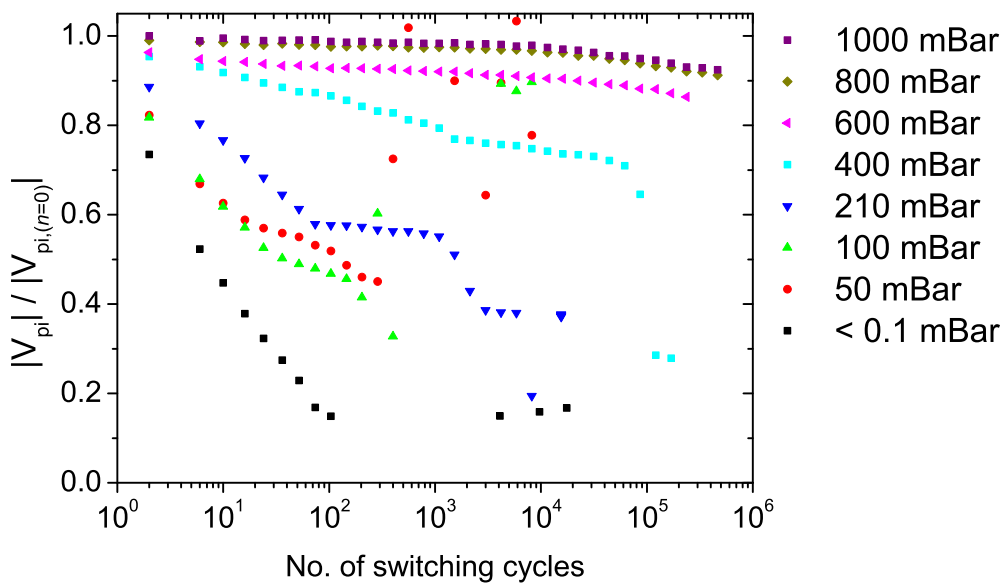

Figure 3.19: Degradation of $V_{\mathrm{pi}}$ as a function of the number of switching cycles for pressures between 0 and $1000 \mathrm{mBar}$. Actuation voltage is $35 \mathrm{~V}$.

Pressure, design and actuation voltage thus all have a significant influence on transient behavior as well as mechanical degradation. Producing a product that is both fast enough and reliable enough thus requires careful modeling and measuring to find the right trade-off between these aspects.

\subsection{Conclusions}

In this chapter we discussed how degradation mechanisms can be identified from changes of the $C-V$ curve as a whole. We showed that in addition to homogeneous charging, laterally inhomogeneous charging and mechanical degradation can take place. We then used this to do experiments that specifically target each of the mentioned degradation mechanisms.

Experiments on homogeneous charging showed that the time dependence of the built-in voltage $V_{\text {shift }}$ for shorter timescales can be adequately described by power law dependence. However, experiments on more recent material showed that at longer time scale at higher stress voltage, the results will deviate from power law dependence. In this case the time dependence is better described with a stretched exponential.

Although strong indications of laterally inhomogeneous charging were already obtained from electrical measurements, a more detailed picture could be constructed with Scanning Kelvin Probe Microscopy (SKPM). With this we measured the surface potential as a function of position and time. From the characteristic length scale over which charge moved, we can conclude that injected charge is 
vertically confined and diffuses laterally over time. Also, when we look at the 2D measurement of the surface potential, we see that inhomogeneity arises due to a combination of the topology of the top electrode as well as random variations.

Lastly, experiments involving prolonged rapid cycling of the capacitive switch showed that decreasing the closing time of a capacitive switch by means of actuation voltage, design, or pressure, can greatly increase the mechanical degradation rate. A carefully selected combination of design, pressure, and actuation voltage is needed in order to make a system that is both fast and robust. 


\section{Chapter 4}

\section{Statistical aspects of capacitive switch degradation}

\subsection{Introduction}

In the previous chapter we discussed measurements concerning dielectric charging as well as mechanical degradation. In section 3.3, Fig. 3.7 we saw a spread in the charging rate: for identically designed devices stressed under the same conditions it can differ by more than a factor 4 . In mechanical degradation experiments (section 3.5) we observed a spread as well: again identically designed devices stressed under the same conditions do not exhibit the same degradation rate, especially when it comes to the sudden decrease in $\left|V_{\mathrm{pi}}\right|$ that is observed after an initial gradual decay (Fig. 3.17). In this chapter we will therefore discuss experiments targeted at gaining more insight into how to deal with spread when characterizing device degradation. Furthermore, we will show that when studying the effects of possible improvements in the manufacturing process of capacitive switches it is important to perform a large number of experiments set up in a systematic way.

\subsection{Spread in homogeneous charging rates}

\subsubsection{Measurement procedure}

In order to gain more insight into possible causes of spread in device degradation, new homogeneous charging experiments similar to those in section 3.3 have been 
performed. For these spread characterization experiments capacitive switches as displayed in Fig. 1.1 were picked from all reticle fields on the wafer (excluding partial reticle fields located on the edges). They were stressed with a $70 \mathrm{~V} \mathrm{DC}$ stress voltage. To reduce measurement time, the stressing of a switch was stopped when $V_{\text {shift }}$ became larger than $3 \mathrm{~V}$. The amount of stress time it takes to reach this $3 \mathrm{~V}$ threshold, hereafter referred to as $T_{3 V}$, is used as a measure for the charging rate. In the plotted results the times are normalized by dividing them by the median time to $V_{\text {shift }}=3 \mathrm{~V}$ for typical processing.

\subsubsection{Results}

An example of the $T_{3 V}$ distribution resulting from stressing a wafer processed with recipes that at the time were specified as 'standard processing' is shown in Fig. 4.1. Of course we only get a number of discrete failure times. To plot it, we first need to sort the measured times to $V_{\text {shift }}=3 \mathrm{~V}$ (the ' $x$-values') in ascending order. Then we need to calculate the estimated cumulative probabilities (the ' $y$ values') associated with each element of this sorted array of failure times. For the calculation of the estimated cumulative probability values Bernard's median rank estimator is used [68]:

$$
C(i)=\frac{i-0.3}{N+0.4}
$$

where $C(i)$ is the cumulative probability belonging to the $i^{\text {th }}$ element of the (sorted) time to $V_{\text {shift }}=3 \mathrm{~V}$ array, $T_{3 V, i}$, and $N$ the number of devices that were measured. Counting of the $T_{3 V}$ array starts with $i=1$. By using this method, a less biased ranking is obtained than with for instance $C(i)=i / N$, which overestimates the failure fraction as a function of failure time, or $C(i)=(i-1) / N$, which underestimates the failure fraction as a function of failure time.

As can be seen from the data, the $95 \%$ interval spans approximately one decade of time to $V_{\text {shift }}=3 \mathrm{~V}$. This implies that when we study the effect of for instance a different nitride composition, if we only measure a few devices for each process variation, the effect will only be visible if it is very large (multiple orders of magnitude slower or faster charging), or we will have to measure the charging distribution for each process variation.

If we assume that process variations are negligible over short distances, it is possible to find out if the spread on charging is intrinsic or process-related by comparing the spread between neighboring devices to the spread over the wafer. This is shown in Fig. 4.2, where $T_{3 V}$ is plotted as a function of $T_{3 V}$ of a neighboring device right next to it. It is done for several different proprietary nitride variations.

From the observed charging rates of neighboring devices we can clearly see that charging rates of neighboring devices are correlated and that differences in charging rate are small over short distances. This correlation is observed for all nitride compositions. Closer inspection of the data will reveal that the spread (in 


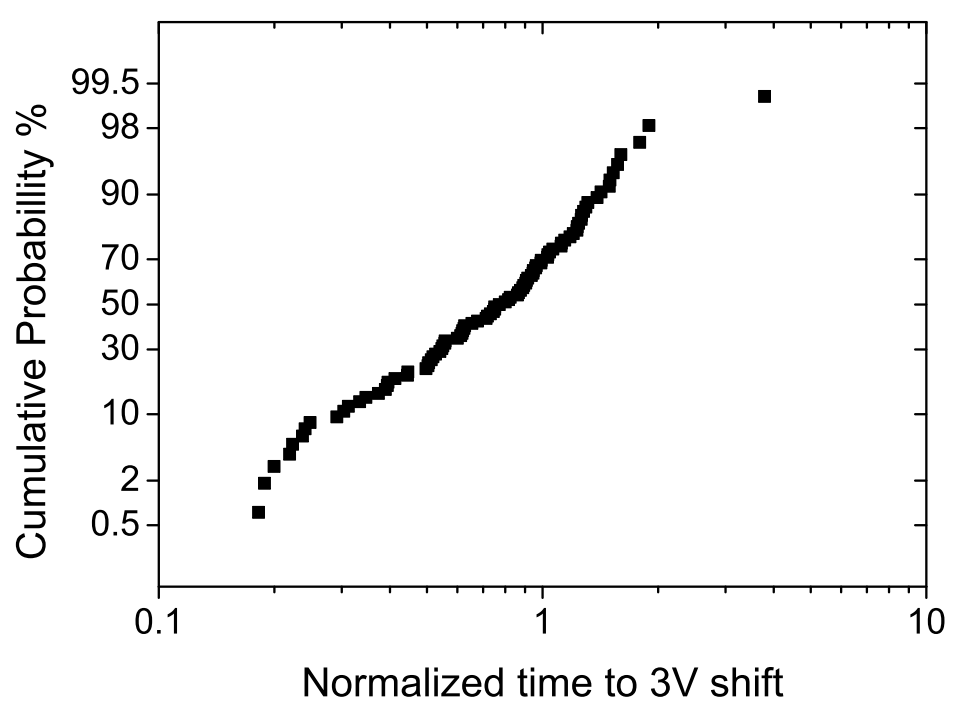

Figure 4.1: Cumulative distribution of the stress time it takes to induce a $V_{\text {shift }}$ of $3 \mathrm{~V}$.

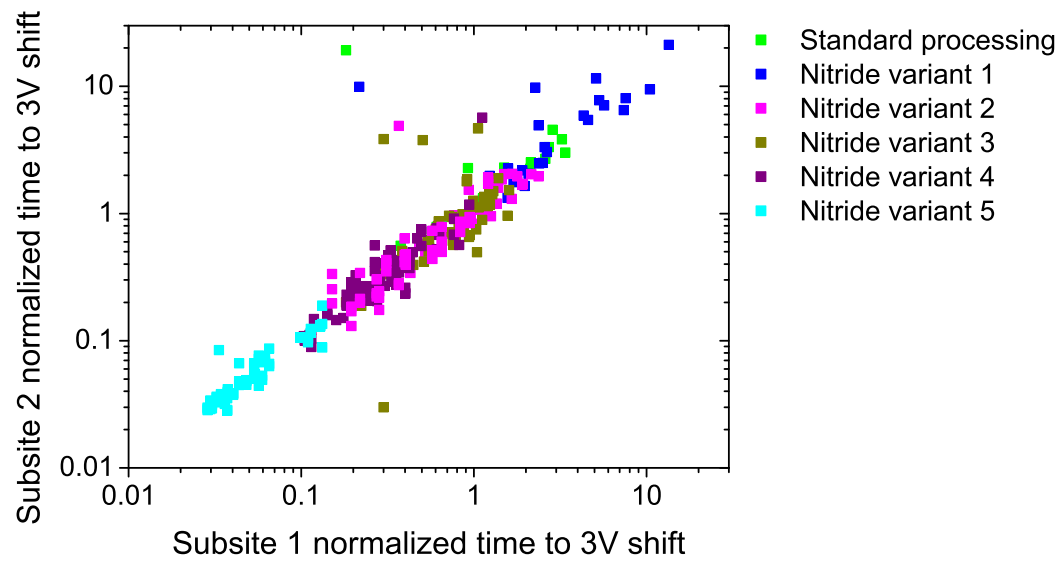

Figure 4.2: Stress time it takes to induce a $V_{\text {shift }}$ of $3 \mathrm{~V}$ as a function of the time for neighboring devices. 

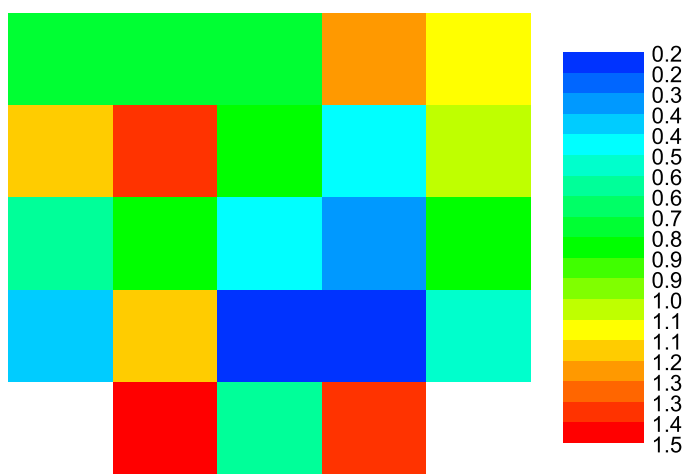

Figure 4.3: Wafermap of the normalized time to $V_{\text {shift }}=3 \mathrm{~V}$. Each value is the average of 4 neighboring devices.

decades) between neighboring devices is 2.8 times smaller than wafer level spread. This shows that variations in the charging rate in Fig. 4.1 are mostly processing related. Variations are the results of slow gradients of material properties across the wafer, and not due to intrinsic randomness in the properties of the dielectric. Because of this, neighboring devices are more alike, than devices further apart. A wafermap with the normalized time to $V_{\text {shift }}=3 \mathrm{~V}$ (averaged over neighboring devices) is shown in Fig. 4.3. Here we see that although no clear linear gradient or ring-shape pattern is visible, there is clustering of data. An example of this is the predominantly blue-green region in the lower-right part of the map, and the yellow-orange portion at the top-right corner.

Examples of possible causes of spread are thickness variations and variations in dielectric composition. To check if the most obvious reason for variation in charging rate, i.e. the thickness of the dielectric, is responsible for the observed spread, we tried to correlate the time to $V_{\text {shift }}=3 \mathrm{~V}$ with the closed state capacitance of each individual device. This is shown in Fig. 4.4. From the data we see that $C_{\text {close }}$ and the logarithm of the time to $V_{\text {shift }}=3 \mathrm{~V}$ are only weakly correlated $(r=-0.1886)$.

Although the observed correlation is statistically significant (a $96.2 \%$ confidence level that the two are negatively correlated), the amount of correlation is by no means strong enough to conclude that the observed long-range wafer level spread is indeed chiefly due to dielectric thickness variation. There are multiple possible reasons for this. For instance, there can be another source of processing spread which causes a significant part of the observed spread in charging rates. Also, the measured $C_{\text {close }}$ does not only depend on the dielectric thickness, but also greatly depends on the remaining air gap, which is determined by the contact mechanics, and exact area of the electrodes. The remaining air gap and exact 


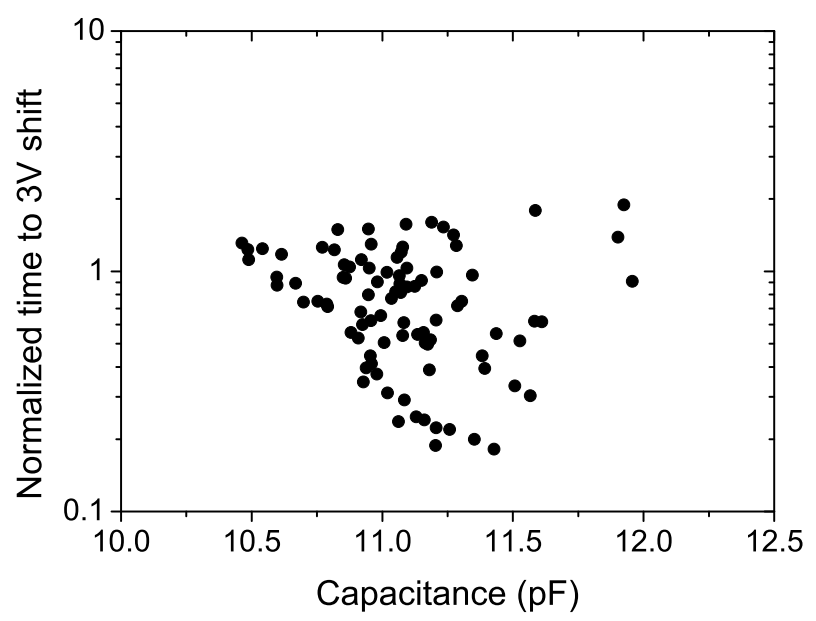

Figure 4.4: Normalized time to $V_{\text {shift }}=3 \mathrm{~V}$ as function of the closed state capacitance.

area of the electrode vary from device to device and can overshadow variation in dielectric thickness, thereby reducing the expected correlation between $C_{\text {close }}$ and the charging rate.

Because spread is so clearly caused by long-range processing related fluctuations, its nature will be determined by the used equipment, processing conditions, and the way the particular wafer was handled. Therefore we can't just assume the cause of variation, whatever it may be, is distributed normally. Because of this, we also don't know what distribution can be expected for the $T_{3 V}$ results. However, we would still like to know how the data would be distributed if the long-range variations could be solved, as it can give information about the remaining spread as well as the underlying degradation mechanism.

Since the correlation between the charging rate and $C_{\text {close }}$ is only weak compared to the observed correlation between neighboring devices, we re-normalize the measured $T_{3 V}$ of each device to the local time expected from neighboring devices, instead of scaling it with a certain $C_{\text {close }}^{\gamma}$. This normalization time $T_{3 V \text {,local }}$ for such a cluster of devices was calculated as:

$$
T_{3 V, \text { local }}=\exp \left(\frac{1}{N} \sum_{i=1}^{N} \log \left(T_{3 V, i}\right)\right),
$$

where $N$ is the amount of neighboring devices within a reticle field (in our case $N=4$ ), and $T_{3 V, i}$ the time to $V_{\text {shift }}=3 \mathrm{~V}$ of device $i$. Note that although this way of normalizing the data is in anticipation of a log-normal distribution, it does not differ very much from standard averaging, and will not force a non-log-normal 


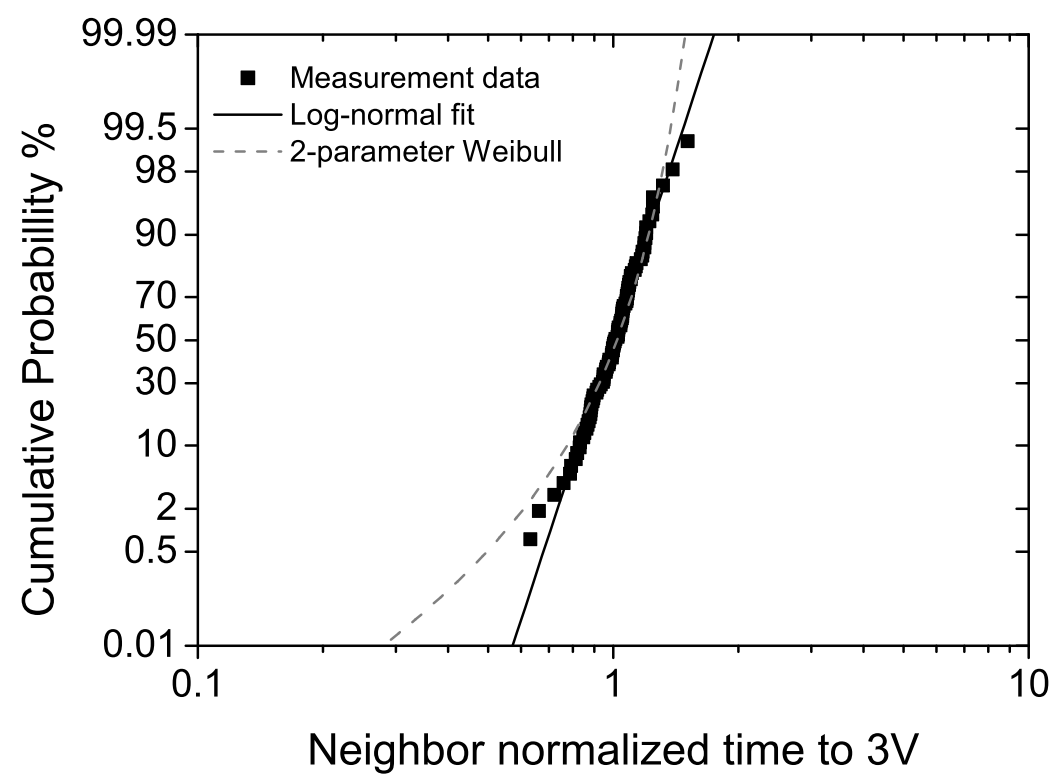

Figure 4.5: Stress time it takes to induce a $V_{\text {shift }}$ of $3 \mathrm{~V}$ as a function of the time to $V_{\text {shift }}=3 \mathrm{~V}$ normalized to the local time to $V_{\text {shift }}=3 \mathrm{~V}$ calculated from neighboring devices.

distribution toward a log-normal one. The resulting distribution we obtain in this way is shown in Fig. 4.5. As can be seen from the data, the $95 \%$ interval now spans only 0.36 decade, i.e. 2.8 times less spread.

By doing the renormalization, we eliminate the effects of long-range gradients that are equipment and handling specific. Because of this, the remaining spread will be more random in nature. Hence, while the overall wafer variation might not be distributed normally, it is reasonable to assume that the remaining spread after renormalization is distributed in a way closer to the normal distribution. Because of this, it is now warranted to fit likely degradation distributions to the data.

Fig. 4.5 shows two of such commonly used reliability distributions fitted to the data: a log-normal and 2-parameter Weibull. Fitting was done using the maximum likelihood method [68]. Maximizing the Log-likelihood sum for this was done in Mathematica. Although both fit reasonably well, a $\chi^{2}$ test shows with $98 \%$ certainty that log-normal gives a better description than 2-parameter Weibull. This not surprising given the fact that the failure criterion we used was the time needed to let the gradual process of dielectric charging induce a $3 \mathrm{~V}$ shift in the $C$ - $V$ curve, and did not use a failure criterion linked to a weakest-link like process. Weakest-link like processes typically lead to a Weibull distribution, while wear-out 
mechanisms in semiconductors (dielectrics can be viewed as a semiconductors with a very large band gap) typically lead to a log-normal distribution [16].

Another important thing to note is that the observed correlation between neighboring devices can be used when studying the influence of stress variations on device degradation behavior to reduce the effects of processing variations. An example of this is the stress voltage dependence of the charging behavior. By stressing devices that are close to each other, the stressed devices are more alike (i.e. 2.8 times less spread), so that the measurements will result in a better picture of how the charging rate increased with stress voltage. This was already used in section 3.3, where the results we showed in Fig. 3.8 ( $V_{\text {shift }}$ as a function of time for stress voltages between $60 \mathrm{~V}$ and $85 \mathrm{~V}$ ), were obtained from devices that were all located in the same reticle field. When we looked at the fit parameters $k$ of this data (Fig. 3.10), we saw monotonously increasing values that do only deviate up to 0.15 decades from the possible models. From the spread results in Fig. 4.1 we would normally expect much greater deviations. The results in Fig. 3.7 were obtained earlier, when the correlation between neighboring devices was not yet known. Here the voltage dependence of the charging rate could only be fitted well because of the large amount of devices that were measured.

Although the correlation between $C_{\text {close }}$ and $T_{3 V}$ was only weak, we saw that this could be explained by variations in the remaining air gap and the exact plate area. Due to this, no definite answer could be given whether the wafer level spread is mainly due to variation in dielectric thickness. However there is a second way by which we can check if it is plausible. We can use the results of Fig. 3.7 to check if the spread we observed in Fig. 4.1 can be explained by thickness variation alone, if we assume that the voltage acceleration of the charging rate we found is an electric field-driven process i.e. that doubling the thickness of the dielectric has the same result as halving the voltage. A small variation $\Delta t_{E}$ in the effective electric thickness $t_{E}$ (i.e. combination of dielectric thickness and the dielectric permitivity) of the dielectric will then result in the following change in rate constant $k$ of the stretched exponential:

$$
k=\alpha \exp \left(\beta t_{E} \frac{V}{t_{E}+\delta t_{E}}\right) \simeq \alpha \exp \left(\beta V\left(1-\frac{\Delta t_{E}}{t_{E}}\right)\right) .
$$

To see how this impacts the time to $V_{\text {shift }}=3 \mathrm{~V}$, which we define as $T_{3 V}$ we solve 
Eq. 3.21 for $3 \mathrm{~V}$ :

$$
\begin{aligned}
3 & =V_{\max } \cdot\left(1-\exp \left(-k T_{3 V}^{\delta}\right)\right) \\
k T_{3 V}^{\delta} & =-\log \left(1-\frac{3}{V_{\max }}\right) \\
T_{3 V} & =\left(\frac{-\log \left(1-\frac{3}{V_{\max }}\right)}{k}\right)^{\frac{1}{\delta}} .
\end{aligned}
$$

Combining Eq. 4.3 and Eq. 4.6 gives us:

$$
T_{3 V}=\frac{\left(-\log \left(1-\frac{3}{V_{\max }}\right)\right)^{\frac{1}{\delta}}}{\alpha^{\frac{1}{\delta}} \exp \left(\frac{\beta V}{\delta}\left(1-\frac{\Delta t_{E}}{t_{E}}\right)\right)}=\frac{\left(-\log \left(1-\frac{3}{V_{\max }}\right)\right)^{\frac{1}{\delta}}}{\alpha^{\frac{1}{\delta}} \exp \left(\frac{\beta V}{\delta}\right)} \exp \left(\frac{\beta V}{\delta} \frac{\Delta t_{E}}{t_{E}}\right) .
$$

With the results of Fig. $3.7(\beta=0.106, \delta=0.4)$, and a stress voltage $V=70 \mathrm{~V}$, the observed spread for the total wafer can be explained if $\Delta t_{E}$ has a $95 \%$ interval of $11 \%$ of the average $\Delta t_{E}$. This is not an unreasonable figure given the fact that during the etching of the sacrificial materials, a small portion of the dielectric will also be removed, as the etchant is not $100 \%$ selective with respect to $\mathrm{Si}_{\mathrm{x}} \mathrm{N}_{\mathrm{y}}: \mathrm{H}$. This will introduce additional spread in the dielectric thickness.

From Eq. 4.7 we can also see that when $\Delta t_{E}$ has a normal distribution, $T_{3 V}$ has a log-normal distribution. This is in agreement with the fit result of Fig. 4.5, where we saw that log-normal gives a better fit than 2-parameter Weibull.

All this means that reducing wafer level processing spread of processing steps involving the dielectric is very important to make devices that have less spread in the charging rate. Together with the observed spread in charging rates, we can conclude from the correlation results that there is a lot of room for improvement.

In Fig. 4.6 the distribution of the time to $V_{\text {shift }}=3 \mathrm{~V}$ is shown for three different variations of the dielectric composition. Several wafers were tested for each variant. Here we see clear differences between the three variants: dielectric variant 1 has a median charging rate that is almost 1.5 orders of magnitude higher than that of the standard dielectric, while variant 2 has an approximately $0.5-1$ orders of magnitude lower median charging rate. There is also a large overlap between the standard dielectric and variant 2: the top $25 \%$ of the standard dielectric charges at approximately the same rate as the bottom $25 \%$ of variant 2 . This means that if we had only measured a few devices, differences in charging rate could have easily been masked or enhanced by the spread in charging rate, thereby leading to wrong conclusions. In case only one device from the standard wafer and one device of dielectric variant 2 would have been measured, there is a chance of approximately $8 \%$ that the device with the standard dielectric has a higher time to $V_{\text {shift }}=3 \mathrm{~V}$ than the device with dielectric variant 2 . 


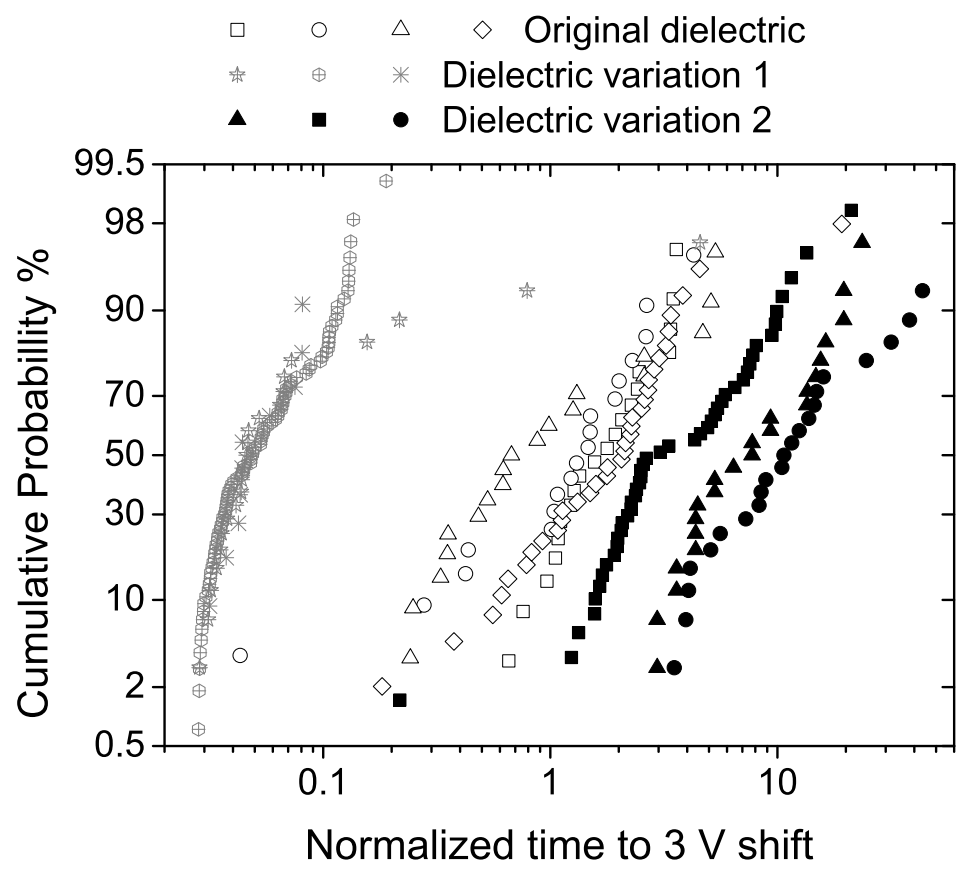

Figure 4.6: Cumulative distribution of the stress time it takes to induce a $V_{\text {shift }}$ of $3 \mathrm{~V}$ for three different variations of the dielectric composition, with several wafers tested for each variant (each symbol belongs to a particular wafer). 


\subsubsection{Conclusions on spread in homogeneous charging}

By measuring the charging behavior of a large amount of devices, each under the same stress conditions, the charging rate distribution was obtained for several wafers. A spread of about one order of magnitude for a $95 \%$ interval was found for individual wafers. The spread of charging rates (in decades) for neighboring devices was a factor 2.8 smaller. The neighbor-renormalized results were distributed lognormally, which is in agreement with the notion that charging is a gradual, and not a weakest-link like process. We also calculated that if wafer level spread is primarily caused by variations in the electric field strength, as a first order approximation the effective electric thickness (i.e. combination of dielectric thickness and the dielectric permitivity) has to have a spread of about $11 \%$, again for the $95 \%$ interval.

Although the width from the charging distribution is important from a yield perspective in and of itself, from the charging distributions we can also conclude that if we want to characterize the influence of stress intensity (for instance voltage or temperature), we either have to measure the charging rate distribution at each stress intensity, or measure devices that are close to each other at each stress intensity. This neighbor correlation was already used in the previous chapter to save measurement time.

When studying the effect of the impact of changes in the manufacturing process, for example changes in dielectric composition or deposition conditions, it is not possible to use the correlation of neighboring devices. Because of this it is important to measure the charging rate distributions over the whole wafer before making any conclusions about its effects. We investigated the effect of dielectric composition on charging rate, and while we saw clear differences in the distributions of the time to $V_{\text {shift }}=3 \mathrm{~V}$, there is a large overlap of the distributions. This means that measuring too few devices can lead to wrong conclusions.

\subsection{Spread in mechanical degradation}

In section 3.5 Fig. 3.17 we saw that when a device is stressed in a way that it mechanically deforms, two phases can be observed in the degradation of $\left|V_{\mathrm{pi}}\right|$ : initially it only degrades gradually, but after a certain critical amount of degradation a sudden large decrease in $\left|V_{\mathrm{pi}}\right|$ is observed. In this section we will go into more detail regarding the differences in the failure distributions of the initial gradual degradation phase and the subsequent sudden large decrease in $\left|V_{\mathrm{pi}}\right|$.

\subsubsection{Measurement procedure}

For the following measurements, 46 devices were picked that are similar to the ones used in section 3.5. We applied rapid pulses $(60 \mathrm{~V})$ and measured the change in $\left|V_{\mathrm{pi}}\right|$ as a function of the number of switching cycles. The interval between two $C-V$ 


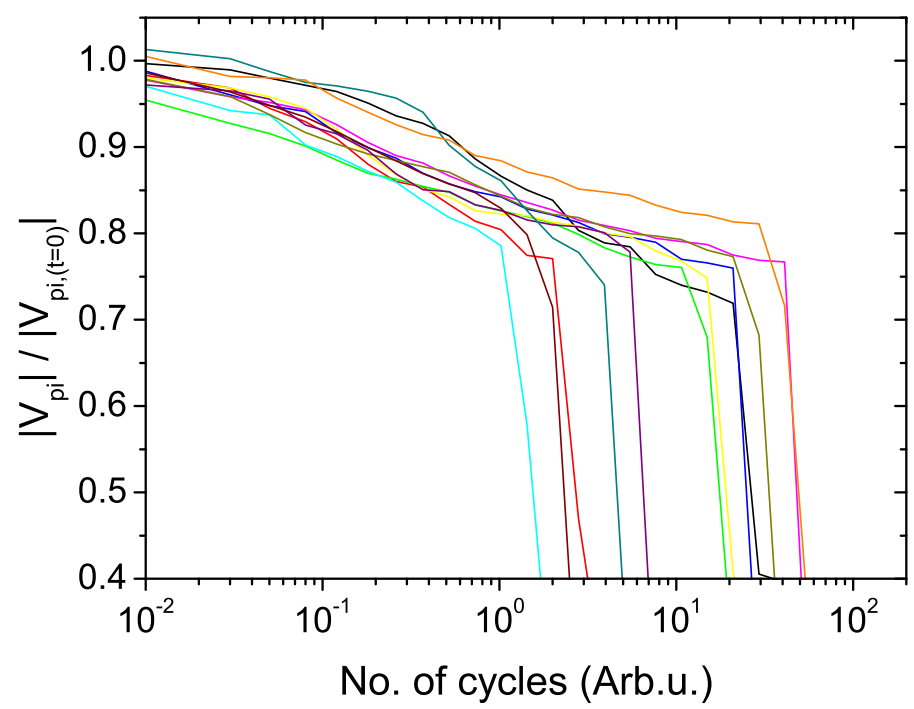

Figure 4.7: $\left|V_{\mathrm{pi}}\right|$ as a function of the number of switching cycles for a selection of devices. $V_{\text {act }}=60 \mathrm{~V}$

curves increased exponentially. Because during the measurement of the $C-V$ curve the switch closes with a closing voltage just a fraction above pull-in, the effect of the $C-V$ curve is insignificant with respect to the applied closing voltage. From these degradation data we extracted the amount of switching cycles $N_{\text {threshold }}$ it takes to induce various percentages of reduction of $\left|V_{\mathrm{pi}}\right|$.

\subsubsection{Measurement results}

Fig. 4.7 shows the degradation of $\left|V_{\mathrm{pi}}\right|$ as a function of the number of switching cycles for a selection of the measured devices. For clarity a representative subset of 12 out of 46 devices are shown. Just as in section 3.5, we see an initial gradual degradation phase, followed at a certain point by a sudden drop in $\left|V_{\mathrm{pi}}\right|$.

For the extraction of $N_{\text {threshold }}$ linear interpolation was used to get a better estimate. However, if degradation exhibits a sharp jump in $\left|V_{\mathrm{pi}}\right|$, it is unclear after how many cycles $\left|V_{\text {pi }}\right|$ degraded more than the set threshold. We defined a sharp jump as a change of more than $10 \%$ between the two measurement points around the threshold. In this case interpolation is no longer a valid way for determining $N_{\text {threshold. }}$ For this data we only know the interval in which the threshold was passed. We took value it had just before passing the threshold (left side of the interval) for use in cumulative probability plots. Later in this section, when we 


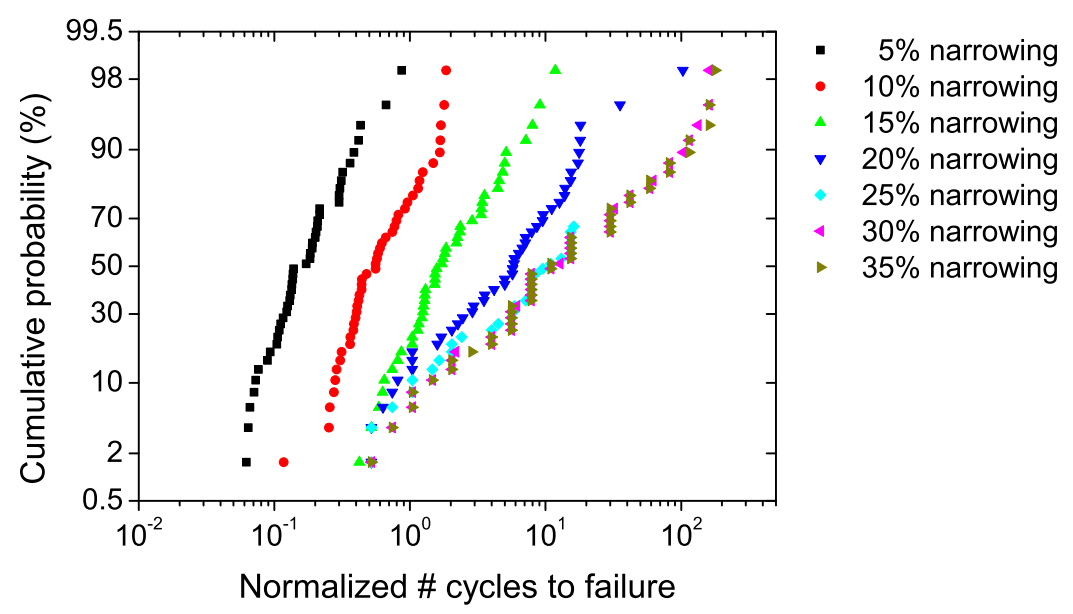

Figure 4.8: Cumulative distribution of the number of switching cycles it takes to induce various amounts of reduction in $\left|V_{\mathrm{pi}}\right|$.

will determine what kind of distribution best fits the resulting degradation distributions, we take the whole interval into account, i.e. we use interval censoring [68] for these measurement points. For that data the difference in the cumulative distribution function (CDF) at the time just before and just after passing the threshold is used in the Log-likelihood sum.

When we use the procedure to extract $N_{\text {threshold }}$ for the measured devices with various thresholds for the amount of narrowing, we obtain Fig. 4.8. For low narrowing thresholds $(5-15 \%)$, the curves are approximately parallel. Around $20 \%$ the distribution becomes wider, especially for the lower end of the distribution. At a threshold of $25 \%$ and above, the distribution is much wider than for the lower thresholds, and increasing the threshold does not significantly increase the median $N_{\text {threshold }}$ anymore.

From the change in the width of the degradation distribution around a threshold of $20 \%$ we can conclude that for a threshold beyond this value an additional source of spread is introduced. The source of the extra spread can be identified when we look at Fig. 4.7 again: we can see that the value of $\left|V_{\mathrm{pi}}\right| /\left|V_{\mathrm{pi},(t=0)}\right|$ just before the sudden drop varies from device to device. This spread in critical mechanical deformation in this case results in the observed widening of the distribution of $N_{\text {threshold }}$.

Note that this widening could change if the critical mechanical deformation and the initial gradual degradation are correlated. In that case the threshold dependence of the amount of spread on $N_{\text {threshold }}$ could be enhanced (if devices degrading slowly during the gradual phase tend to have a larger critical deformation), or re- 


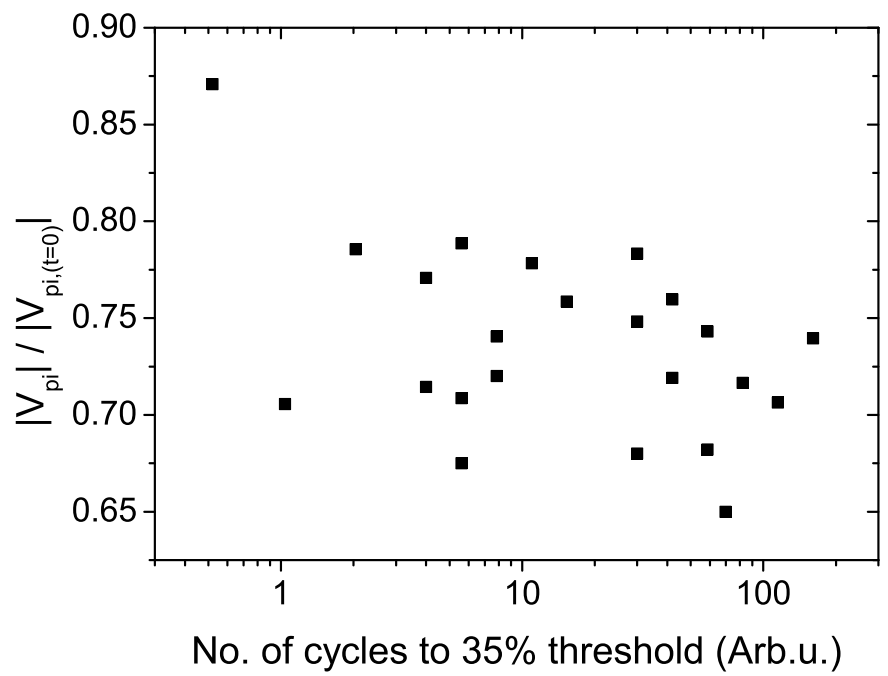

Figure 4.9: $N_{\text {threshold }}$ as a function of $\left|V_{\mathrm{pi}}\right| /\left|V_{\mathrm{pi},(t=0)}\right|$ just before reaching the threshold. No clear correlation is observed between the two.

duced (if devices degrading slowly during the gradual phase tend to have a smaller critical deformation). However, in this experiment no clear correlation between $N_{\text {threshold }}$ and the value of $\left|V_{\mathrm{pi}}\right| /\left|V_{\mathrm{pi},(t=0)}\right|$ just before reaching the threshold was observed, as can be seen in Fig. 4.9.

We also observed that when $\left|V_{\mathrm{pi}}\right|$ suddenly decreases, this does not mean the device completely sticks. The open state capacitance can increase by more than $50 \%$, but the $C$ - $V$ curve does not deform so much that pull-in and pull-out can no longer be observed. This is shown in Fig. 4.10, where the $C$ - $V$ curve of a device is shown as it was before stress, just before the critical mechanical deformation, and after the sudden drop in $\left|V_{\mathrm{pi}}\right|$. From the change in the open state capacitance it is readily concluded that crossing the critical mechanical deformation is accompanied by a large mechanical change (the gap can be reduced by more than $33 \%$ ). We also see changes in the closed state capacitance, which we believe is due to changes in the contact mechanics resulting in a smaller closed state gap. This will the subject of future studies on capacitive switch reliability.

In section 4.2 we saw that the charging rate of neighboring devices strongly correlated. For the mechanical degradation experiments we also looked if neighboring devices have a similar number of cycles to the defined failure criterion. This is shown in Fig. 4.11. From this figure we can see that neighboring devices correlate much weaker, especially for a $5 \%$ narrowing threshold. The observed spread in degradation therefore is due to microscopic fluctuations instead of the more 


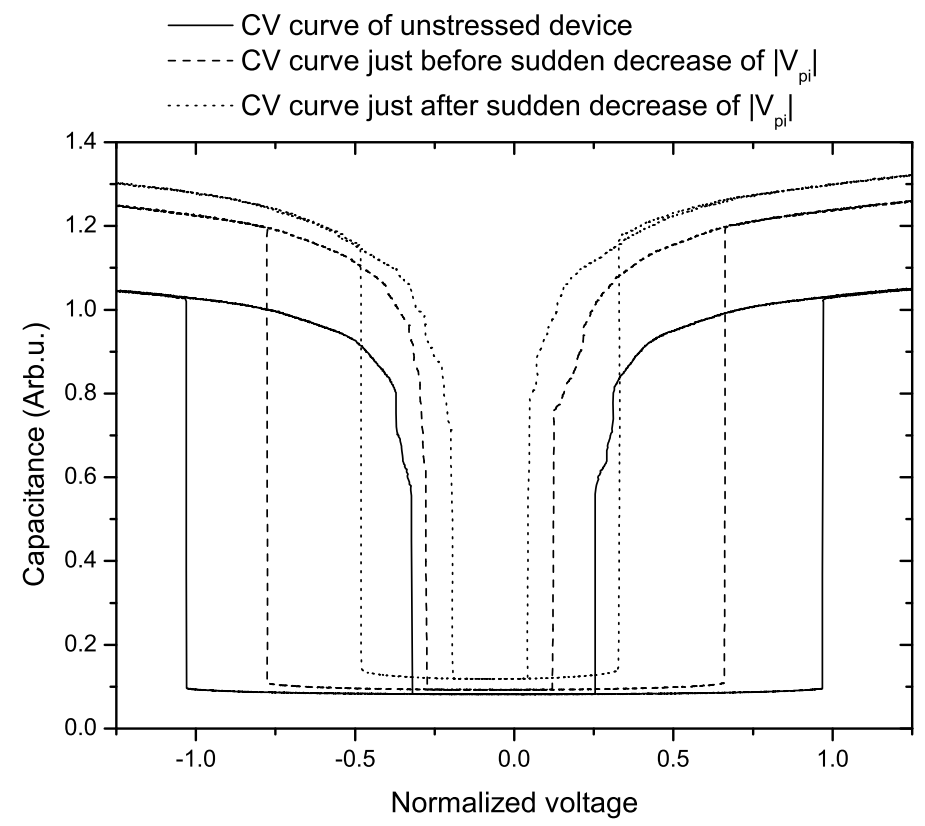

Figure 4.10: $C-V$ curve at different stages of mechanical degradation. In the initial phase the $C$ - $V$ curve changes only gradually. After the sudden decrease of $\left|V_{\mathrm{pi}}\right|$, the open state capacitance also has increased significantly. 


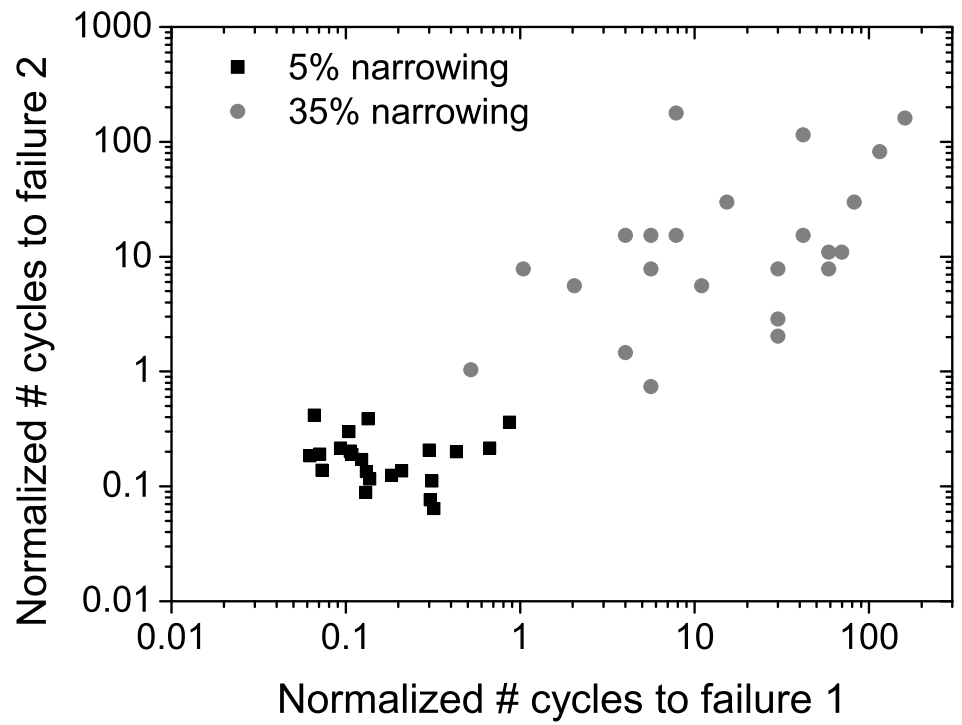

Figure 4.11: Normalized number of switching cycles to induce various amounts of reduction in $\left|V_{\mathrm{pi}}\right|$ for neighboring devices.

long-range gradients in device properties we saw for the dielectric charging results.

Apart from the above qualitative evaluation of the measurement data, Maximum Likelihood fitting was used to determine whether a Weibull or a log-normal gives the best description of the data. Overall, log-normal resulted in better fits than Weibull. However, due to the large spread, a $\chi^{2}$ test on the Log Likelihood sum did only justify choosing log-normal over Weibull for lower thresholds. At thresholds higher than $25 \%$ no significant difference was observed. An example of the difference of the two fits is given in Fig. 4.12. Given the fact that the sudden jump in degradation might well be governed by weakest-link statistics, it can be expected that for higher threshold the failures become Weibull distributed. More data is required to prove if this indeed is the case.

\subsubsection{Conclusions on spread in mechanical degradation}

Mechanical degradation tests were performed on 46 devices at an actuation voltage of $60 \mathrm{~V}$. The width of the distribution of the number of cycles that are needed to reach a threshold percentage of narrowing greatly depended on the chosen narrowing threshold. This indicates that spread in mechanical degradation is not only dependent on differences in degradation rate during the gradual degradation phase, but also on the critical mechanical degradation a device can suffer before it will 


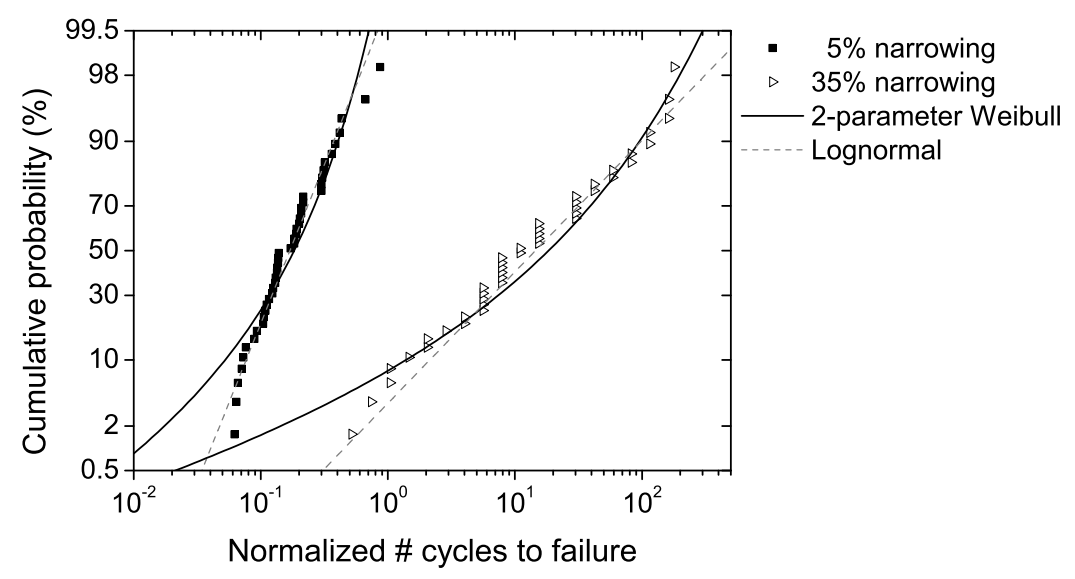

Figure 4.12: Cumulative distribution of the number of switching cycles it takes to induce $5 \%$ and $35 \%$ reduction in $\left|V_{\mathrm{pi}}\right|$ with a 2-parameter Weibull and log-normal distribution fitted to the measurement data.

show a sudden large decrease in $\left|V_{\mathrm{pi}}\right|$. The sudden decrease in $\left|V_{\mathrm{pi}}\right|$ is accompanied by a large increase in the open state capacitance.

\subsection{Conclusions}

In this chapter we discussed the impact of spread on the characterization of degradation in capacitive switches. We saw that when homogeneous dielectric charging is characterized to aide the development of a reliable dielectric, it is important to measure the distribution of charging rates, because differences between two differently processed wafers can be easily masked by spread if only a few devices are measured. In principle, the same is also true when measuring the voltage dependence of dielectric charging, however, if the observed close correlation of the charging rate of neighboring devices is used, it is possible to study this with relatively few measurements.

The study we did on mechanical degradation showed us that the spread in the amount of switching cycle needed for a set percentage of $\left|V_{\mathrm{pi}}\right|$ reduction, can greatly depend on the chosen threshold. This is due to the two distinct phases that were observed in mechanical degradation: first an initial gradual graduation, followed by a rapid degradation of the switch after a certain critical amount of mechanical degradation, which varies from device to device.

All in all, taking statistical aspects of MEMS degradation mechanisms into account increases the confidence in and prevent erroneous interpretation of obtained results. This makes it vital to investigate distributions of MEMS degradation mech- 
anisms, not only for qualification of a MEMS process, but also earlier on when the process is still in development. 


\section{Chapter 5}

\section{Conclusions and recommendations}

\subsection{Conclusions}

At the start of this study on degradation mechanisms in RF MEMS capacitative switches, most literature only discussed (homogeneous) dielectric charging, or did not specify which aspect of MEMS degradation they studied. Also, no clear methodology for stressing and characterizing degradation was available, although the way trapped charge influence the MEMS were already modeled in a comprehensive way $[21,35]$.

In the beginning of the reliability research project, only a slow test setup was available, which lead to a closer investigation of how homogeneous dielectric charging can best be characterized. We showed that measuring only the center of the $C$ - $V$ curve to extract $V_{\text {shift }}$ dramatically reduces the effect the measurement has on the device (section 2.2). When $V_{\text {shift }}$ is measured in this way it will also not be influenced by $C$ - $V$ curve narrowing. All this makes it an attractive method to characterize homogeneous dielectric charging. This new method was presented at the ICMTS '06 [22], on which we later elaborated in a journal article [69].

Although the center shift method made it possible to study dielectric charging using a relatively slow measurement setup, the decision was made to develop a more versatile setup for RF MEMS characterization (section 2.3). The setup, a custom single frequency 1-port S-parameter measurement system, made it possible to measure capacitances with a sub-microsecond time resolution a femto-farad range capacitance resolution, which is significantly faster than the IF bandwidth of high-end off-the-shelf Vector Network Analyzers. This made it possible to measure closing and opening transients, and to measure a whole $C-V$ curve within less than 
half a second.

With the upgraded setup it became possible to do systematic, fast, and accurate measurements on a large number of devices. Results showed that for material available at the time, dielectric charging exhibited a $\sqrt{t}$ time dependence. These results were presented at the IRPS '07 [23], which to our knowledge is the largest published set of measurements on dielectric charging to date. Later, not yet published results (see section 3.3) showed that this was a special case of a more general stretched exponential time dependence. Although no final conclusions can be made, together with the results of reliability experiments on similar materials [49], the results make a good case for the hypothesis that dielectric charging is governed by charge trapping in current induced metastable defects, which is a mechanism also held responsible for changes in the conductivity of silicon nitride MIM capacitors.

The enhanced test setup also enabled us to measure the whole $C-V$ curve with minimal influence on the device. We used this capability to build on the work by Rottenberg [35] and develop and test a framework to distinguish mechanical degradation from laterally inhomogeneous charging by looking at the relation between $V_{\mathrm{pi}}$ and $V_{\mathrm{po}}$ of degrading devices (section 3.2). We showed that the two degradation mechanisms can and do both occur in RF MEMS capacitive switches, and can be identified using our method. This was presented at the MEMS '08 conference [45].

Apart from the effect laterally inhomogeneous charging has on the $C$ - $V$ curve, we also got the first direct proof of this phenomenon by using Scanning Kelvin Probe Microscopy to directly measure the surface potential of a charged dielectric as function of position and time (section 3.4). We observed that the trapped charges were vertically confined, and could directly observe lateral charge diffusion. Using timed measurements we were able to extract an estimate for the diffusion coefficient. The results were presented at the IRPS '08 conference [70].

After we showed that mechanical degradation can occur, we studied it in more detail, and characterized how mechanical degradation depends on the design and the circumstances under which the devices are used (section 3.5). Pressure, design, and actuation voltage all greatly influence the degradation of capacitive switches because of the effects they have on the closing transient. Careful modeling and measuring is required to find the right trade-offs between these aspects.

As already mentioned, we measured a large number of devices during our study of MEMS degradation mechanisms. As in all fabrication processes, spread was observed in the rates of the degradation mechanisms. In chapter 4 we saw that it is important to measure whole distributions of degrading devices when studying the effects processing and stress conditions have on degradation mechanisms. We saw in section 4.2 that the spread in charging rate can easily mask the differences between two differently processed wafers. In the mechanical degradation results of section 4.3 we could see from the measured distributions that mechanical degradation consists of two phases. Initially the devices only gradually degrade. At a certain point, a critical amount of mechanical degradation is induced, after which the 
device will show a phase of rapid degradation. Due to this behavior the spread in the amount of cycles needed to cross a certain amount of mechanical degradation greatly depends on the chosen threshold.

With all these results we can paint the following picture about degradation of RF MEMS capacitive switches. When a device is in use (not necessarily fully in contact $[41,43])$, degradation will cause changes in the $C$ - $V$-curve. If the $C$ - $V$ curve only shifts, this is due to laterally homogeneous charging. If the $C$ - $V$-curve also narrows, this narrowing can be due to laterally inhomogeneous charging, mechanical degradation, or a combination thereof. By looking how the pull-in and pull-out voltage change, it can be determined what is the main contribution to the narrowing effect. If a small change in $\left(V_{\mathrm{pi}}^{+}-V_{\mathrm{pi}}^{-}\right)$is accompanied by a relatively large change in $\left(V_{\mathrm{po}}^{+}-V_{\mathrm{po}}^{-}\right)$the narrowing is mainly due to laterally inhomogeneous charging. If the change in $\left(V_{\mathrm{po}}^{+}-V_{\mathrm{po}}^{-}\right)$is smaller than the change in $\left(V_{\mathrm{pi}}^{+}-V_{\mathrm{pi}}^{-}\right)$, narrowing is mainly due to mechanical degradation.

When we look at the underlying mechanisms, we now know that for the dielectrics we have investigated, dielectric charging under constant voltage stress has a stretched exponential time dependence, where the charging rate depends exponentially on the stress voltage. Due to the layout of the top electrode, but also due to random variations in the properties of the dielectric and electrodes, charge will be injected laterally inhomogeneous. Dielectric charging effects can recover again by charge leaking away. Additionally, laterally inhomogeneous charging effects can also recover due to lateral diffusion of the trapped charge. The timescale on which this diffusion takes place is now known, and can serve as input for a model describing degradation and recovery. For now mechanical degradation is a more complicated matter, where we have identified many factors that can play a role in the sensitivity to degradation, but do not know yet how this can best be modeled.

All in all, we now have clear methodologies for stressing and characterizing degradation, and gained insight as to how results obtained with using these methodologies are to be interpreted. These are used on a daily basis at EPCOS Netherlands B.V. for aiding the development of a commercially viable process for making $\mathrm{RF}$ MEMS capacitive switches.

\subsection{Recommendations}

Although significant advances have been made with respect to knowledge of RF MEMS degradation mechanisms, a lot remains to be done. In this section we therefore give a brief, non-exhaustive list of topics that need further study.

First of all, only RF MEMS degradation has been studied for this thesis, and in order to say more about RF MEMS reliability, several questions need to be answered. A very important one is the question of how much degradation can be tol- 
erated before a device becomes unusable. This greatly depends on the application, and answering this question requires cooperation between designers (on product, circuit, and device level), process developers, and reliability engineers. Related to this is the question of what kind of stress does the device have to endure. Again this greatly depends on the application.

Another important issue with respect to reliability is the identification of spread sources. The reason for this is that although a typical device might be very reliable, if the spread in degradation rate is high, a lot of devices on the lower end of the scale won't be reliable, so that only a low yield can be achieved. Minimizing spread is therefore important for reliability and yield.

On the degradation mechanisms side, there also remains work to be done. Although a lot has been done on characterization and identification of degradation mechanism, achieving a deeper understanding of the fundamental reasons behind the mechanisms is still very desirable. Modeling of dielectric charging and mechanical degradation could then lead to a more predictable behavior of device degradation under various operating conditions.

Finally, we would like to mention here that a device which is used will not only degrade over time, it can also recover. We already saw this in section 3.4 and 3.2.2. Studying recovery mechanism can be useful for gaining a better understanding of how the device degrades, and can also result in a better estimate of the expected lifetime of a device under the operating conditions set by the application. 


\section{Bibliography}

[1] G. M. Rebeiz, RF MEMS - Theory, Design, and Technology. John Wiley \& Sons, Inc, 2003, pp. 1-20.

[2] H. De Los Santos, G. Fischer, H. Tilmans, and J. van Beek, "RF MEMS for Ubiquitous Wireless Connectivity. Part II. Application," Microwave Magazine, IEEE, vol. 5, pp. 50-65, 2004.

[3] H. M. R. Suy, R. W. Herfst, P. G. Steeneken, J. Stulemeijer, and J. Bielen, "The static behavior of RF MEMS capacitive switches in contact," in Proceedings of Nanotech MSM (Modeling and Simulation of Microsystems) 2008, Jun. 2008.

[4] G. M. Rebeiz, RF MEMS - Theory, Design, and Technology. John Wiley \& Sons, Inc, 2003, pp. 36-38.

[5] H. M. R. Suy, H. G. A. Huizing, P. G. Steeneken, and O. I. Yanson, "A Compact Scalable Circuit Model for RF MEMS Switches," in Proceedings of Nanotech MSM 2007, vol. 3, 2007, pp. 65-68.

[6] E. K. Chan, E. C. Kan, and R. W. Dutton, "Nonlinear dynamic modeling of micromachined microwave switches," in 1997 IEEE MTT-S International Microwave Symposium Digest, Jun. 1997, pp. 1511-1514.

[7] J. B. Muldavin and G. M. Rebeiz, "Nonlinear electro-mechanical modeling of MEMS switches," in 2001 IEEE MTT-S International Microwave Symposium Digest, vol. 3, May 2001, pp. 2119-2122.

[8] R. Sattler and G. Wachutka, "Compact Models for Squeeze-Film Damping in the Slip Flow Regime," in Proceedings of 2004. 24th International Conference on Microelectronics, vol. 1, May 2004, pp. 71-78.

[9] D. Mercier, P. Blondy, D. Cros, and P. Guillon, "An electromechanical model for MEMS switches," in 2001 IEEE MTT-S International Microwave Symposium Digest, vol. 3, 2001, pp. 2123-2126. 
[10] J. Bielen and J. Stulemeijer, "Efficient electrostatic-mechanical modeling of C-V curves of RF-MEMS switches," in Proceedings of Eurosime200\%, Apr. 2007.

[11] M. Andrews, I. Harris, and G. Turner, "A comparison of squeeze-film theory with measurements on a microstructure," Sensors and Actuators, vol. 36, pp. 79-87, 1993.

[12] P. G. Steeneken, T. G. S. M. Rijks, J. T. M. van Beek, M. J. E. Ulenaers, J. De Coster, and R. Puers, "Dynamics and squeeze film gas damping of a capacitive RF MEMS switch," J. Micromech. Microeng., vol. 15, pp. 176-184, 2005.

[13] W. S. Griffen, H. H. Richardson, and S. Yamanami, "A study of fluid squeezefilm damping," J. Basic Eng. Trans. ASME, pp. 451-456, 1966.

[14] J. J. Blech, "On isothermal squeeze films," J. Lubrication Tech., vol. 105, pp. 615-620, Oct. 1983.

[15] T. Veijola, H. Kuisma, and J. Lahdenpera, "Model for gas film damping in a silicon accelerometer," in Proceedings of 1997 International Conference on Solid-State Sensors and Actuators, vol. 4, Jun. 1997, pp. 1097-1100.

[16] M. Ohring, Reliability and Failure of Electronic Materials and Devices. Academic Press, 1998.

[17] B. Stark, editor, "MEMS Reliability Assurance Guidelines for Space Applications," National Aeronautics and Space Administration (NASA), and Jet Propulsion Laboratory (JPL), California Institute of Technology, Pasadena, California, USA, Tech. Rep., 1999.

[18] G. M. Rebeiz, RF MEMS - Theory, Design, and Technology. John Wiley \& Sons, Inc, 2003, pp. 185-192.

[19] S. Melle, E. Flonrens, D. Dubuc, K. Grenier, P. Pons, F. Pressecq, L. Kuchenbecker, J. L. Muraro, L. Bary, and R. Plana, "Reliability overview of RF MEMS devices and circuits," in Proceedings of $33^{\text {rd }}$ European Microwave Conference, vol. 1, Oct. 2003, pp. 37-40.

[20] C. Goldsmith, J. Ehmke, A. Malczewski, B. Pillans, S. Eshehnan, Z. Yao, J. Brank, and M. Eberly, "Lifetime characterization of capacitive RF MEMS switches," in 2001 IEEE MTT-S Int. Microwave Symp. Digest, vol. 1, May 2001, pp. 227-230.

[21] W. M. van Spengen, R. Puers, R. Mertens, and I. De Wolf, "A comprehensive model to predict the charging and reliability of capacitive RF MEMS switches," J. Micromech. Microeng., vol. 14, pp. 514-521, 2004. 
[22] R. W. Herfst, H. G. A. Huizing, P. G. Steeneken, and J. Schmitz, "Characterization of dielectric charging in RF MEMS capacitive switches," in Proceedings of 2006 IEEE International Conference on Microelectronic Test Structures, Mar. 2006, pp. 133-136.

[23] R. W. Herfst, P. G. Steeneken, and J. Schmitz, "Time and voltage dependence of dielectric charging in RF MEMS capacitive switches," in Proceedings of $45^{\text {th }}$ Annual IEEE International Reliability Physics Symposium, 2007, pp. 417-421.

[24] I. Wibbeler, G. Heifer, and M. Hietschold, "Parasitic charging of dielectric surfaces in capacitive microelectromechanical systems (MEMS)," Sensors and Actuators A: Physical, pp. 74-80, Nov. 1998.

[25] X. Yuan, J. C. M. Hwang, and D. F. C. L. Goldsmith, "Modeling and characterization of Dielectric-Charging Effects in RF MEMS Capacitive Switches," in 2005 IEEE MTT-S Int. Microwave Symp. Digest, 2005, pp. 753-756.

[26] G. Papaioannou, M.-N. Exarchos, V. Theonas, G. Wang, and J. Papapolymerou, "Temperature Study of the Dielectric Polarization Effects of Capacitive RF MEMS Switches," IEEE Transactions on Microwave Theory and Techniques, vol. 53, no. 11, p. 3467, Nov. 2005.

[27] J. R. Reid, "Dielectric charging effects on capacitive MEMS actuators," in 2002 IEEE MTT-S Int. Microwave Symp. Digest, RF MEMS workshop, Jun. 2002 .

[28] S. S. McClure, L. D. Edmonds, R. Mihailovich, A. H. Johnston, P. Alonzo, J. DeNatale, J. Lehman, and C. Yui, "Radiation effects in microelectromechanical systems (MEMS): RF Relays," IEEE Trans. On Nuclear Science, vol. 49, no. 6, pp. 3197-3202, Dec. 2002.

[29] J. R. Reid, "Capacitive Switch Reliability Issues," in Government Microelectronics Applications Conference (GOMAC) 2002 Digest, Monterey, CA, Mar. 2002 .

[30] R. L. Borwick III, P. A. Stupar, J. F. DeNatale, R. Anderson, and R. Erlandson, "Variable MEMS capacitors implemented into RF filter systems," IEEE Transactions on Microwave Theory and Techniques, vol. 51, Issue 1, pp. 315-319, 2003.

[31] M. R. Douglass, "Lifetime estimates and unique failure mechanisms of the DigitalMicromirror Device (DMD)," in Proceedings of $36^{\text {th }}$ Annual IEEE International Reliability Physics Symposium, 1998, pp. 9-16. 
[32] E. K. Chan, K. Garikipati, and R. W. Dutton, "Characterization of contact electromechanics through capacitance-voltage measurements and simulations," J. Microelectromechanical Systems, vol. 8, no. 2, pp. 208-217, Jun. 1999.

[33] W. M. van Spengen, P. Czamecki, R. Puers, J. T. M. van Beek, and I. De Wolf, "The influence of the package environment on the functioning and reliability of RF-MEMS switches," in Proceedings of $43^{\text {rd }}$ Annual IEEE International Reliability Physics Symposium, Apr. 2005, pp. 337-341.

[34] X. Yuan, S. Cherepko, J. Hwang, C. L. Goldsmith, C. Nordquist, and C. Dyck, "Initial Observation and Analysis of Dielectric-Charging Effects on RF MEMS Capacitive Switches," in 2004 IEEE MTT-S International Microwave Symposium Digest, 2004, pp. 1943-1946.

[35] X. Rottenberg, B. Nauwelaers, W. De Raedt, and H. A. C. Tilmans, "Distributed dielectric charging and its impact on RF MEMS devices," in Proceedings of $34^{\text {th }}$ European Microwave Conference - Amsterdam, 2004, pp. 77-80.

[36] W. van Spengen, R. Puers, R. Mertens, and I. D. Wolf, "A low frequency electrical test set-up for the reliability assessment of capacitive RF MEMS switches," Journal of Micromechanics and Microengineering, vol. 13, no. 5, pp. 604-612, 2003.

[37] H. Nieminen, J. Hyyrylinen, T. Veijola, T. Ryhnen, and V. Ermolov, "Transient capacitance measurement of MEM capacitor," Sensors and Actuators A, vol. 117, pp. 267-272, 2005.

[38] A. Technologies, "Applying Error Correction to Network Analyzer Measurement, Agilent AN 1287-3," www.agilent.com.

[39] — - "Understanding the Fundamental Principles of Vector Network Analysis, Agilent AN 1287-1," www.agilent.com.

[40] J. R. Reid and R. T. Webster, "Measurements of charging in capacitive microelectromechanical switches," ELECTRONICS LETTERS, vol. 38, no. 24, pp. 1544-1545, Nov. 2002.

[41] P. Czarnecki, X. Rottenberg, P. Soussan, P. Ekkels, P. Muller, P. Nolmans, W. De Raedt, H. A. C. Tilmans, R. Puers, L. Marchand, and I. De Wolf, "Influence of the substrate on the lifetime of capacitive RF MEMS switches," in Proceedings of $21^{\text {st }}$ IEEE International Conference on Micro Electro Mechanical Systems, Jan. 2008, pp. 172-175. 
[42] P. Czarnecki, X. Rottenberg, P. Soussan, P. Nolmans, P. Ekkels, P. Muller, H. A. C. Tilmans, W. De Raedt, R. Puers, L. Marchand, and I. De Wolf, "New insights into charging in capacitive RF MEMS switches," in Proceedings of $21^{\text {st }}$ IEEE International Conference on Micro Electro Mechanical Systems, Apr. 2008, pp. 496-505.

[43] P. Czarnecki, X. Rottenberg, P. Soussan, P. Ekkels, P. Muller, P. Nolmans, W. D. Raedt, H. A. C. Tilmans, R. Puers, L. Marchand, and I. D. Wolf, "Effect of substrate charging on the reliability of capacitive RF MEMS switches," Sensors and Actuators A, 2008.

[44] X. Rottenberg, I. D. Wolf, B. Nauwelaers, W. D. Raedt, and H. Tilmans, "Analytical Model of the DC Actuation of Electrostatic MEMS Devices With Distributed Dielectric Charging and Nonplanar Electrodes," J. Microelectromechanical Systems, vol. 16, no. 5, pp. 1243-1253, Oct. 2007.

[45] R. W. Herfst, P. G. Steeneken, and J. Schmitz, "Identifying degradation mechanisms in RF MEMS capacitive switches," in Proceedings of $21^{\text {st }}$ IEEE International Conference on Micro Electro Mechanical Systems, 2008, pp. 168-171.

[46] P. Lorrain, D. R. Corson, and F. Lorrain, Electromagnetic Fields and Waves, $3^{\text {rd }}$ edition. W. H. Freeman and Company, New York, 1987, p. 116.

[47] S. P. Lau and J. M. Shannon, "Generation and annealing kinetics of current induced metastable defects in amorphous silicon alloys," Journal of NonCrystalline Solids, vol. 266-269, pp. 432-436, 2000.

[48] J. M. Shannon, S. C. Deane, B. McGarvey, and J. N. Sandoe, "Current induced drift mechanism in amorphous $S_{i} N_{x}: H$ thin film diodes," Appl. Phys. Lett., vol. 65, Dec. 1994.

[49] M. H. W. M. van Delden and P. J. van der Wel, "Reliability and electric properties for PECVD $A-S i N_{x}: H$ films with an optical bandgap ranging from 2.5 to $5.38 \mathrm{eV}$," in IEEE 41 ${ }^{\text {st }}$ Annual International Reliability Physics Symposium, Dallas, Texas, 2003, pp. 293-297.

[50] R. A. Street, "Current-induced defect creation and recovery in hydrogenated amorphous silicon," Appl. Phys. Lett., vol. 59, no. 9, pp. 1084-1086, Aug. 1991.

[51] M. Stutzmann, W. B. Jackson, and C. C. Tsai, "Light-induced metastable defects in hydrogenated amorphous silicon: A systematic study," Phys. Rev. $B$, vol. 32 , pp. 23-47, 1985.

[52] D. L. Staebler and C. R. Wronski, "Reversible conductivity changes in discharge-produced amorphous Si," Appl. Phys. Lett., vol. 31, p. 292, 1977. 
[53] D. Redfield and R. H. Bube, "Reinterpretation of degradation kinetics of amorphous silicon," Appl. Phys. Lett., vol. 54, no. 11, pp. 1037-1039, 1989.

[54] S. Zafar, B. H. Lee, J. Stathis, A. Callegari, and T. Ning, "A Model for Negative Bias Temperature Instability (NBTI) in Oxide and High $\kappa$ pFETs," in Proceedings of 2004 Symposium an VLSI Technology, 2004, pp. 208-209.

[55] J. Kim, E. T. Ogawa, and J. W. McPherson, "Time Dependent Dielectric Breakdown Characteristics of Low-k Dielectric (SiOC) Over a Wide Range of Test Areas and Electric Fields," in Proceedings of $45^{\text {th }}$ Annual IEEE International Reliability Physics Symposium, 2007, pp. 399-404.

[56] M. Nonnenmacher, M. P. O’Boyle, and H. K. Wickramasinghe, "Kelvin probe force microscopy," Appl. Phys. Lett., vol. 58, pp. 2921-2923, Jun. 1991.

[57] M. Lamhamdi, L. Boudou, P. Pons, J. Guastavino, A. Belarni, M. Dilhan, Y. Segui, and R. Plana, "Si3N4 thin films proprerties for RF-MEMS reliability investigation," in Proceedings of the $14^{\text {th }}$ International Conference on SolidState Sensors, Actuators and Microsystems, Jun. 2007, pp. 579-582.

[58] A. Belarni, M. Lamhamdi, P. Pons, L. Boudou, J. Guastavino, Y. Segui, G. Papaioannou, and R. Plana, "Kelvin probe microscopy for reliability investigation of RF-MEMS Capacitive Switches," Microelectronics Reliability, vol. 48, no. 8-9, pp. 1232-1236, 2008.

[59] H. J. Wintle, "Surface conduction on insulators: Analysis and interpretation of the Faraday cage experiment," J. Appl. Phys., vol. 81, no. 6, pp. 2682-2685, 1997.

[60] H. J. Wintle and M. P. Pépin, "Decay of surface charge between electrodes on insulator surfaces," J. of Electrostatics, vol. 48, pp. 115-126, 2000.

[61] J.-H. Park, "Separation of surface and bulk electrical conductivity in $\mathrm{KH}_{2} \mathrm{PO}_{4}$ and $\mathrm{KH}_{2} \mathrm{AsO}_{4}$ crystals at high temperatures," Solid State Communications, vol. 123, pp. 291-294, 2002.

[62] J.-H. Park and B.-C. Choi, "Electrical conductivity and impedance characteristics of $\mathrm{RbH}_{2} \mathrm{PO}_{4}$ crystal above room temperature," Materials Letters, vol. 57, pp. 2162-2167, 2003.

[63] C. Cismaru and J. L. Shohet, "Synchrotron radiation-induced surfaceconductivity of $\mathrm{SiO}_{2}$ for modification of plasma charging," Appl. Phys. Lett., vol. 76, no. 16, pp. 2191-2193, 2000.

[64] C. H. Ha, J. K. Kim, and K.-W. Whang, "The role of the defect levels in MgO in the low firing voltage, wide driving voltage margin operation of an alternate current plasma display panel," J. Appl. Phys., vol. 101, p. 123301, 2007. 
[65] Y. Hashimoto and T. Sakakibara, "Effect of Surface Current on Photoelectron Emission from Mica," Electrical Engineering in Japan, vol. 133, no. 1, 2000.

[66] G. H. Buh, H. J. Chung, and Y. Kuk, "Real-time evolution of trapped charge in a $\mathrm{SiO}_{2}$ layer: An electrostatic force microscopy study," Applied Physics Letters, vol. 79, no. 13, pp. 2010-2012, 2001.

[67] P. H. Holloway and H. J. Stein, "Quantitative Detection of Oxygen in Silicon Nitride on Silicon," J. Electrochem. Soc.: SOLID-STATE SCIENCE AND TECHNOLOGY, vol. 123, no. 5, pp. 723-728, 1976.

[68] P. A. Tobias and D. C. Trindade, Applied reliability. Chapman \& Hall/CRC, 1995.

[69] R. W. Herfst, H. G. A. Huizing, P. G. Steeneken, and J. Schmitz, "Center-shift method for the characterization of dielectric charging in RF MEMS capacitive switches," IEEE Transaction on Semiconductor Manufacturing, pp. 148-153, 2008 .

[70] R. W. Herfst, P. G. Steeneken, J. Schmitz, A. J. G. Mank, and M. van Gils, "Kelvin probe study of laterally inhomogeneous dielectric charging and charge diffusion in RF MEMS capacitive switches," in Proceedings of $46^{\text {th }}$ Annual IEEE International Reliability Physics Symposium, 2008, pp. 492-495. 


\section{Summary}

In this study, degradation mechanisms in Radio Frequency Micro-Electromechanical systems (RF MEMS) capacitive switches have been investigated. We presented a methodology to characterize device degradation in a way that minimizes the influence of the measurement on the device itself, even if relatively slow equipment is used. An automated fast RF-CV measurement setup was developed, which enabled us to measure a whole $C-V$ curve with minimal influence on the device.

With the setup a wide array of systematic, fast and accurate degradation experiments were done. With these experiments we gained more insight in the time dependence of laterally homogeneous dielectric charging. Also, it enabled us to develop a framework to distinguish mechanical degradation from laterally inhomogeneous dielectric charging, two totally different degradation mechanisms which, despite their differences, have similar effects on the device. The framework also allowed us to choose stress patterns that specifically target mechanical degradation. This was used to investigate the influence of various operating conditions on mechanical degradation.

The phenomenon of laterally inhomogeneous dielectric charging was also studied more directly with Scanning Kelvin Probe Microscopy. The SKPM measurements also showed that trapped charge diffuse in the lateral direction. This contributes to the recovery effects we observed with electrical measurements.

Finally, due to the level of automation of the developed measurement setup, and the availability of enough measurement material, it was possible to do a large number of measurements. This allowed us to study how spread in device degradation impacts the way characterization of those degrading devices should be done. We saw that measuring whole distributions of degrading devices is important when studying the various mechanisms that contribute to degradation of RF MEMS capacitive switches, as differences in device degradation due to for instance a change in stress intensity can easily be masked by the spread in degradation rates. 


\section{Samenvatting}

Dit proefschrift behandelt de studie is verricht op het terrein van degradatie mechanismes in Radio Frequentie Micro Electro-Mechanische Systemen (RF MEMS) capacitatieve schakelaars. We hebben een methodologie gepresenteerd waarmee component degradatie kan worden gekarakteriseerd op een manier waarbij de invloed van de meting op het component zelf wordt geminimaliseerd, zelfs als relatief langzame apparatuur wordt gebruikt.

Met de meetopstelling zijn een grote verscheidenheid aan systematische, snelle en nauwkeurige degradatie-experimenten verricht. Met deze experimenten hebben we meer inzicht verkregen in de tijdsafhankelijkheid van lateraal-homogene oplading van het dielectricum in RF MEMS capacitatieve schakelaars. Ook stelde het ons in staat om methodes te ontwikkelen waarmee mechanische degradatie kan worden onderscheiden van lateraal-inhomogene oplading. Dit zijn twee totaal verschillende degradatie-mechanismes die, ondanks het grote verschil, soortgelijke effecten hebben op een capacitatieve schakelaar. Tevens stelden deze methodes ons in staat experimenten te doen die specifiek gericht waren op mechanische degradatie. Dit is gebruikt om de invloed op mechanische degradatie van variaties in de manier waarop de componenten worden bedreven te bestuderen.

Ook is lateraal-inhomogene oplading van het dielectricum op een meer directe wijze bestudeerd: met Scanning Kelvin Probe Microscopy (SKPM). Deze SKPM metingen toonden aan dat ingevangen lading in laterale richting diffunderen. Dit draagt bij aan de herstel effecten die zijn waargenomen met elektrische metingen.

Ten slotte, het automatiseringsniveau van de meetopstelling en de beschikbaarheid van genoeg materiaal om te meten maakten het mogelijk een groot aantal metingen te doen. We hebben bestudeerd hoe spreiding in de degradatie van componenten de manier waarop de karakterisatie ervan moet worden gedaan. We zagen dat bij het bestuderen van de verschillende mechanismes die bijdragen aan de degradatie van RF MEMS capacitatieve schakelaars het belangrijk is hele distributies van degraderende componenten te meten, aangezien verschillen in degradatie door bijvoorbeeld een verandering in de stressintensiteit eenvoudig kunnen worden gemaskeerd door spreiding in degradatie snelheden. 


\section{List of publications}

J. Stulemeijer, and R.W. Herfst, "Chaos in electrostatically actuated RF-MEMS measured and modeled", Accepted for publication in Proceedings of $22^{\text {nd }}$ IEEE International Conference on Micro Electro Mechanical Systems, 2009.

H. M. R. Suy, R. W. Herfst, P. G. Steeneken, J. Stulemeijer, and J. Bielen, "The static behavior of RF MEMS capacitive switches in contact", in Proceedings of Nanotech MSM (Modeling and Simulation of Microsystems) 2008, Jun. 2008.

R. W. Herfst, H. G. A. Huizing, P. G. Steeneken, and J. Schmitz, "Centershift method for the characterization of dielectric charging in RF MEMS capacitive switches", IEEE Transaction on Semiconductor Manufacturing, 2008, pp. 148-153.

R. W. Herfst, P. G. Steeneken, J. Schmitz, A. J. G. Mank, and M. van Gils, "Kelvin probe study of laterally inhomogeneous dielectric charging and charge diffusion in RF MEMS capacitive switches", in Proceedings of $46^{\text {th }}$ Annual IEEE International Reliability Physics Symposium, 2008, pp. 492-495.

R. W. Herfst, P. G. Steeneken, and J. Schmitz, "Identifying degradation mechanisms in RF MEMS capacitive switches", in Proceedings of $21^{\text {st }}$ IEEE International Conference on Micro Electro Mechanical Systems, 2008, pp. 168-171.

R. W. Herfst, P. G. Steeneken, and J. Schmitz, "Time and voltage dependence of dielectric charging in RF MEMS capacitive switches", in Proceedings of $45^{\text {th }}$ Annual IEEE International Reliability Physics Symposium, 2007, pp. 417-421. 
R. W. Herfst, H. G. A. Huizing, P. G. Steeneken, and J. Schmitz, "Characterization of dielectric charging in RF MEMS capacitive switches", in Proceedings of 2006 IEEE International Conference on Microelectronic Test Structures, Mar. 2006, pp. 133-136. 


\section{Dankwoord}

Zoals te verwachten valt bij een promotie-onderzoek in een vakgebied als RF-MEMS reliability, is dit niet in je eentje mogelijk. Velen hebben mij op allerlei manieren geholpen, en die wil ik hier dan ook bedanken. Natuurlijk draagt de een wat meer, en de ander wat minder bij, en ik wil hier enkele personen in het bijzonder noemen.

Allereerst natuurlijk mijn promotor, Jurriaan Schmitz, die in de loop van de jaren geholpen heeft bij het schrijven en corrigeren van artikelen, meedacht over de richting van het onderzoek, met goede ideeën kwam en me vooral op een prettige manier heeft weten te motiveren. Ook met Peter Steeneken, mijn directe begeleider gedurende het grootste gedeelte van mijn tijd bij Natlab in Eindhoven, heb ik prettig samengewerkt en ik heb veel van hem geleerd in zowel theoretische als praktische zaken rondom MEMS onderzoek. Verder natuurlijk ook dank aan de rest van de promotie-commissie voor het lezen en goedkeuren van mijn proefschrift.

Ook Reinout Woltjer, Bert Huizing en Andre van Geelen wil ik hier graag noemen. Reinout: bedankt voor het mogelijk maken van de AIO positie bij wat toen nog Philips was. Bert Huizing wil ik bedanken voor zijn inzet voor mijn promotie-onderzoek, eerst als begeleider, en later als groepshoofd. Andre van Geelen: bedankt dat je ondanks de drukte van het opzetten van een nieuw bedrijf je de mogelijkheden hebt gecreëerd waardoor ik na de overname van het RF-MEMS project door EPCOS mijn proefschrift op de nieuwe locatie af heb kunnen maken.

In de loop van de jaren heb ik veel geleerd tijdens de samenwerking met velerlei collega's. Ik wil ze bij deze in min of meer chronologische volgorde noemen. Zo zijn er de mensen van de zogenaamde MEMS-kamer in de groep van Bert Huizing: Hilco Suy, Andre Jansman en Cas van der Avoort. Verder ook Hans Tuinhout en Martijn Goossens, die als critische lezers van mijn concept-artikelen een zeer positieve bijdrage hebben geleverd aan het eindresultaat van de publicaties. Martijn wil ik ook bedanken voor alle praktische hulp in en om de meetfaciliteiten in Eindhoven. Ook ben ik zeker dank verschuldigd aan Arjan Mank, zijn hulp bij de AFM/SKPM metingen heeft een mooie publicatie opgeleverd. Marcel van Gils, Paul van der Wel, Ramon Havens, Jiri Stulemeijer, Arnold den Dekker, Marcel Giessen en Jeroen Bielen: bedankt voor de vele discussies over meetresultaten en MEMS device fysica die mijn begrip van MEMS reliability hebben vergroot. Jeroen 
wil ik in het bijzonder bedanken voor de uitleg die hij mij heeft gegeven op het gebied van statistische technieken.

Verder wil ik ook alle andere collega's die ik bij NXP had, en nu bij EPCOS in Nijmegen heb, bedanken voor de gezellige sfeer waarin ik mijn werk altijd heb kunnen doen. Als laatste, maar zeker niet als minste, wil ik mijn ouders hartelijk bedanken voor de goede zorgen en de liefdevolle steun die ze mij tijdens mijn promotieonderzoek altijd hebben gegeven. 